\title{
Photoacylation of Alcohols in Neutral Medium
}

\author{
Jean-Luc Débieux, ${ }^{\text {[a] }}$ Anne Cosandey, ${ }^{[a]}$ Céline Helgen, ${ }^{[a]}$ and Christian G. Bochet ${ }^{[a]}$
}

\begin{abstract}
We report here conditions which allow the photoacylation of primary, secondary and tertiary alcohols with $N$-acetyl-5,7dinitroindoline under exceptionally mild conditions, at wavelengths harmless to most functional groups, including otherwise photosensitive ones.
\end{abstract}

\section{Introduction}

The transformation of an alcohol into an ester is one of the most important reactions in organic synthesis, as attested by the countless preparative methods published to date. ${ }^{[1,2]}$ However, in many instances, the existing methods require aggressive reagents (acid chlorides, strong bases or acids, activated alkylating agents) and are incompatible with sensitive functional groups. From this viewpoint, the photoinduced acylation of oxygen nucleophiles would represent an attractive alternative. While similar reactions have been successfully developed for the formation of amides and carbamates, ${ }^{[3-6]}$ esters have proven to be more problematic targets, with the notable exception of methyl esters (when methanol is used as the solvent). ${ }^{[7]}$ We report here conditions which allow the photoacylation of primary, secondary and tertiary alcohols with $N$-acetyl-5,7-dinitroindoline under exceptionally mild conditions, at wavelengths harmless to most functional groups, including otherwise photosensitive ones.

\section{Results and Discussion}

Our initial experiments, in which we used typical conditions that are suitable for the formation of amides (1 equiv. each of the nucleophile and acetyldinitroindoline in acetonitrile, irradiation at $350 \mathrm{~nm}$ ), were unsuccessful and led only to deacetylated indoline, acetic acid and unreacted alcohol. Better conversion could only be obtained by using 3 equiv. of the acylating agent. These observations suggested that the photolysis irreversibly produced a species that rapidly decomposed to acetic acid and deacetylated indoline with trace amounts of moisture, if no powerful nu-

[a] Department of Chemistry, University of Fribourg, 9 Chemin du Musée, 1700 Fribourg, Switzerland E-mail: Christian.Bochet@unifr.ch cleophile (such as an amine) was available to react fast enough. A mechanistic study by time-resolved IR spectroscopy indeed showed a transient species with a lifetime of ca. $20 \mu \mathrm{s}^{[8,9]}$

We thus reasoned that anything capable of accelerating the bimolecular process required to form the desired ester would be beneficial. The addition of acyl transfer catalysts (such as $N, N$-4-dimethylaminopyridine) indeed increased the yields (from $22 \%$ to $56 \%$ ). It was finally by increasing the concentration of the reactants, despite reaching saturation of the $N$-acetyl-5,7-dinitroindoline, that consistently good yields were obtained. Hence, irradiation of a $0.1 \mathrm{M}$ solution of $N$-acetyl-5,7-dinitroindoline with 1 equiv. of an alcohol in strictly anhydrous acetonitrile at $375 \mathrm{~nm}$ for $16 \mathrm{~h}$ gave the corresponding acetate in good to excellent yields (Scheme 1, Table 1).

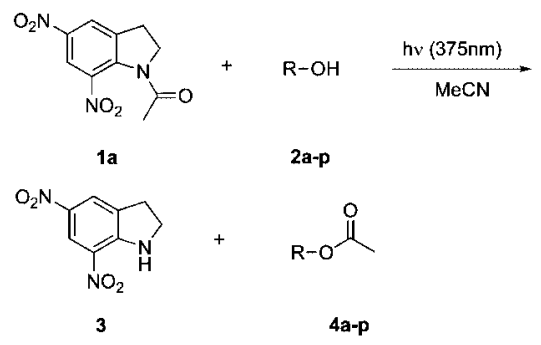

Scheme 1. Acetylation of alcohols with indoline 1a.

The operating wavelength could be increased up to $405 \mathrm{~nm}$, a wavelength where even very UV-photosensitive 3,5-dimethoxybenzoin ester $\mathbf{4 p}$ is stable, without affecting the yields. This adds an interesting facet to our strategy of exploiting chromatic orthogonality ${ }^{[10]}$ because both the esterification and the hydrolysis of $\mathbf{4 p}$ can be carried out photochemically without the need for any external reagent.

The reaction is compatible with the presence of acetyl groups (Entry 13), conjugated and unconjugated alkenes alkynes (Entry 12), and functionalised phenols also react satisfactorily (Entry 7). 
Table 1. Acetylation of alcohols with indoline 1a.

(2)

[a] Determined in situ by ${ }^{1} \mathrm{H}$ NMR spectroscopy. [b] Isolated.

Other groups than simple acetyl can be transferred photochemically. To verify this, we successfully acylated 10 -undecen-1-ol with a series of acylnitroindoles (1a-f), which were prepared in prior work ${ }^{[5]}$ (Scheme 2, Table 2).

We emphasise the simplicity of the experiments, which can be carried out on a normal bench, without the need
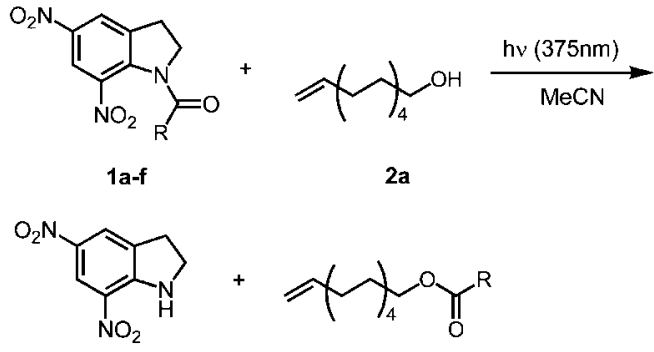

3

4a, 6b-f

Scheme 2. Acylation of alcohols with indolines $\mathbf{1 a}-\mathbf{f}$.

Table 2. Acylation of alcohols with indolines 1a-f.
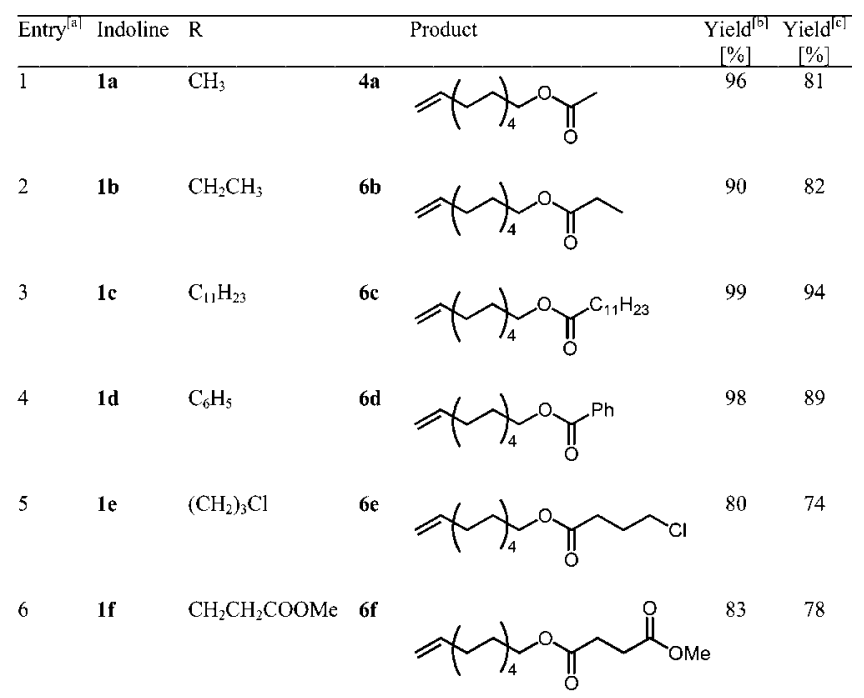

[a] Conditions: $0.1 \mathrm{~m}$ solution in $1 \mathrm{~mL}$ of anhydrous acetonitrile for $16 \mathrm{~h}$, mol ratio of $N$-acyl-5,7-dinitroindoline/alcohol is $1: 1$. [b] Determined by ${ }^{1} \mathrm{H}$ NMR spectroscopy. [c] Isolated yield.

of a mercury lamp, by using a small footprint LED-based photoreactor (Atlas Photonics Inc.) and quartz test tubes, under conventional magnetic stirring.

In conclusion, we extended the use of $N$-acyl-5,7-dinitrondoline for the photoacylation of alcohols with particularly mild conditions and a simple experimental protocol.

\section{Experimental Section}

General Methods: ${ }^{1} \mathrm{H}$ - and ${ }^{13} \mathrm{C}-\mathrm{NMR}$ spectra were recorded with a Fourier transform Bruker-DRX-500 (500 MHz) or Bruker-DPX$360(360 \mathrm{MHz})$ spectrometer with solvent used as a reference. For ${ }^{13} \mathrm{C} \mathrm{NMR}$, the number of hydrogen was determined by a DEPT sequence. IR spectra were recorded with a Fourier transform Mattson 5000 FTIR spectrometer, neat, in $\mathrm{CHCl}_{3}(\mathrm{NaCl}$ cell) or in $\mathrm{KBr}$; absorption bands are in $\mathrm{cm}^{-1}$. UV spectra were recorded with a Perkin Elmer Lambda 40 spectrometer; absorption bands are in nm. EI mass spectra were recorded with an HP 5988A Quadrupol spectrometer, with electron impact $(70 \mathrm{eV})$ and ESI mass spectra with a Bruker FT/MS 4.7 T BioApex II spectrometer. Photochemical irradiations were carried out in a LUMOS 43 photoreactor (Atlas Photonics Inc.), in a quartz vessel, with 1 diode at 375, 385, 
405 or $430 \mathrm{~nm}$, or in a Srinivasan-Griffin (Rayonet-RPR-100) photoreactor, in a quartz vessel, with 16 lamps at $254,300,350$ or $420 \mathrm{~nm}$. Unless otherwise indicated, all commercial reagents were used without further purification.

Typical Procedure for the Preparation of 1-Acyl-5,7-dinitroindolines: A solution of 5,7-dinitroindoline $(43.0 \mathrm{mg}, 0.21 \mathrm{mmol}),{ }^{[6]}$ aluminium trichloride $(68.5 \mathrm{mg}, 0.51 \mathrm{mmol})$ and acid chloride $(0.41 \mathrm{mmol})$ was heated at reflux in 1,2-dichloroethane $(5 \mathrm{~mL})$ for 2 to $6 \mathrm{~h}$. Extraction, followed by trituration in cyclohexane to remove the remaining acid chloride (if not volatile), gave the desired 1-acyl-5,7-dinitroindoline pure or with remaining 5,7-dinitroindoline.

1-Propionyl-5,7-dinitroindoline (1b): Pale brown solid (m.p. 144 $\left.147^{\circ} \mathrm{C}\right) .{ }^{1} \mathrm{H}$ NMR $\left(360 \mathrm{MHz}, \mathrm{CDCl}_{3}\right): \delta=8.58(\mathrm{~s}, 1 \mathrm{H}), 8.26(\mathrm{~s}, 1$ $\mathrm{H}), 4.37(\mathrm{t}, J=8.3 \mathrm{~Hz}, 2 \mathrm{H}), 3.38(\mathrm{t}, J=8.2 \mathrm{~Hz}, 2 \mathrm{H}), 2.56(\mathrm{q}, J=$ $7.3 \mathrm{~Hz}, 2 \mathrm{H}), 1.26(\mathrm{t}, J=7.4 \mathrm{~Hz}, 3 \mathrm{H}) \mathrm{ppm} .{ }^{13} \mathrm{C} \mathrm{NMR}(90.55 \mathrm{MHz}$, $\left.\mathrm{CDCl}_{3}\right): \delta=172.3(\mathrm{C}), 143.1(\mathrm{C}), 139.8(\mathrm{C}), 139.1(\mathrm{C}), 138.1(\mathrm{C})$, $123.0(\mathrm{CH}), 119.8(\mathrm{CH}), 49.9\left(\mathrm{CH}_{2}\right), 29.0\left(\mathrm{CH}_{2}\right), 29.0\left(\mathrm{CH}_{2}\right), 8.7$ $\left(\mathrm{CH}_{3}\right) \mathrm{ppm}$. IR $\left(\mathrm{CHCl}_{3}\right): \tilde{v}=3024,1697,1608,1548,1462,1382$, $1342,1280 \mathrm{~cm}^{-1}$. UV/Vis $(51 \mu \mathrm{M}$ soln in $\mathrm{MeCN}) \lambda\left(\varepsilon, \mathrm{M}^{-1} \mathrm{~cm}^{-1}\right): 204$ (14310), 226 (13140), 353 (11180) nm. MS: $m / z(\%)=265$ (2) $[\mathrm{M}]^{+}, 209$ (90), 163 (7), 117 (9), 89 (8), 57 (100). HRMS: calcd. for $\mathrm{C}_{11} \mathrm{H}_{11} \mathrm{~N}_{3} \mathrm{O}_{5}$ 265.0699; found 265.0674 .

1-Lauroyl-5,7-dinitroindoline (1c): Very pale brown solid, purified from remaining 5,7-dinitroindoline by chromatography (m.p. 105$\left.108{ }^{\circ} \mathrm{C}\right) .{ }^{1} \mathrm{H}$ NMR $\left(360 \mathrm{MHz}, \mathrm{CDCl}_{3}\right): \delta=8.58(\mathrm{~s}, 1 \mathrm{H}), 8.26(\mathrm{~s}, 1$ $\mathrm{H}), 4.37$ (t, $J=8.3 \mathrm{~Hz}, 2 \mathrm{H}), 3.37(\mathrm{t}, J=8.2 \mathrm{~Hz}, 2 \mathrm{H}), 2.51$ (t, $J=$ $7.4 \mathrm{~Hz}, 2 \mathrm{H}$ ), 1.74 (quint, $J=7.5 \mathrm{~Hz}, 2 \mathrm{H}), 1.4-1.2(\mathrm{~m}, 16 \mathrm{H}), 0.88$ $(\mathrm{t}, J=6.8 \mathrm{~Hz}, 3 \mathrm{H}) \mathrm{ppm} .{ }^{13} \mathrm{C} \mathrm{NMR}\left(90.55 \mathrm{MHz}, \mathrm{CDCl}_{3}\right): \delta=$ $171.8(\mathrm{C}), 143.2(\mathrm{C}), 139.8(\mathrm{C}), 139.2(\mathrm{C}), 138.0(\mathrm{C}), 123.0(\mathrm{CH})$, $119.9(\mathrm{CH}), 50.0\left(\mathrm{CH}_{2}\right), 35.7\left(\mathrm{CH}_{2}\right), 31.9\left(\mathrm{CH}_{2}\right), 29.5\left(\mathrm{CH}_{2}\right), 29.4$ $\left(\mathrm{CH}_{2}\right), 29.3\left(\mathrm{CH}_{2}\right), 29.3\left(\mathrm{CH}_{2}\right), 29.1\left(\mathrm{CH}_{2}\right), 28.5\left(\mathrm{CH}_{2}\right), 24.6\left(\mathrm{CH}_{2}\right)$, $22.6\left(\mathrm{CH}_{2}\right), 14.1\left(\mathrm{CH}_{3}\right) \mathrm{ppm}$. IR $\left(\mathrm{CHCl}_{3}\right): \tilde{v}=2927,2855,1695$, $1548,1464,1374,1344,1280 \mathrm{~cm}^{-1}$. UV/Vis $(51 \mu \mathrm{M}$ soln in MeCN): $\lambda\left(\varepsilon, \mathrm{M}^{-1} \mathrm{~cm}^{-1}\right)=226(18240), 349(13922) \mathrm{nm}$. MS: $m / z(\%)=209$ (96), 183 (18), 179 (18), 163 (5), 117 (8), 95 (13), 85 (23), 71 (38), 57 (100). HRMS: calcd. for $\mathrm{C}_{8} \mathrm{H}_{7} \mathrm{~N}_{3} \mathrm{O}_{4}, \mathrm{C}_{12} \mathrm{H}_{23} \mathrm{O} 209.0437,183.1749$; found 209.0437, 183.1749.

1-Benzoyl-5,7-dinitroindoline (1d): Yellow crystals, purified from remaining 5,7-dinitroindoline by recrystallisation (toluene/ethanol, 1:1) (m.p. $\left.193-196{ }^{\circ} \mathrm{C}\right) .{ }^{1} \mathrm{H}$ NMR $\left(360 \mathrm{MHz}, \mathrm{CDCl}_{3}\right): \delta=8.69$ (s, $1 \mathrm{H}), 8.31(\mathrm{~s}, 1 \mathrm{H}), 7.76(\mathrm{~d}, J=7.4 \mathrm{~Hz}, 2 \mathrm{H}), 7.61(\mathrm{t}, J=7.6 \mathrm{~Hz}$, $1 \mathrm{H}), 7.51(\mathrm{t}, J=7.7 \mathrm{~Hz}, 2 \mathrm{H}), 4.39(\mathrm{t}, J=8.2 \mathrm{~Hz}, 2 \mathrm{H}), 3.33(\mathrm{t}, J$ $=8.2 \mathrm{~Hz}, 2 \mathrm{H}) \mathrm{ppm} \cdot{ }^{13} \mathrm{C} \mathrm{NMR}\left(90.55 \mathrm{MHz}, \mathrm{CDCl}_{3}\right): \delta=169.9$ (C), 143.7 (C), 141.3 (C), 139.2 (C), 138.8 (C), 133.3 (C), 132.8 $(\mathrm{CH}), 128.8(\mathrm{CH}), 128.8(\mathrm{CH}), 123.5(\mathrm{CH}), 120.0(\mathrm{CH}), 53.7\left(\mathrm{CH}_{2}\right)$, $29.1\left(\mathrm{CH}_{2}\right) \mathrm{ppm}$. IR (KBr): $\tilde{v}=3100,1661,1600,1545,1528,1439$, $1372,1338,1306 \mathrm{~cm}^{-1}$. UV $(51 \mu \mathrm{M}$ soln in $\mathrm{MeCN}): \lambda\left(\varepsilon, \mathrm{M}^{-1} \mathrm{~cm}^{-1}\right)$ $=229$ (19020), $353(12550) \mathrm{nm}$. MS: $m / z(\%)=313(1)[\mathrm{M}]^{+}, 105$ (100), 77 (31). HRMS: calcd. for $\mathrm{C}_{15} \mathrm{H}_{11} \mathrm{~N}_{3} \mathrm{O}_{5}$ 313.0699; found 313.0701

1-(4-Chlorobutyryl)-5,7-dinitroindoline (1e): Yellow oil, purified from remaining 5,7-dinitroindoline by chromatography. ${ }^{1} \mathrm{H}$ NMR $\left(360 \mathrm{MHz}, \mathrm{CDCl}_{3}\right): \delta=8.59(\mathrm{~s}, 1 \mathrm{H}), 8.28(\mathrm{~s}, 1 \mathrm{H}), 4.42(\mathrm{t}, J=$ $8.4 \mathrm{~Hz}, 2 \mathrm{H}), 3.69(\mathrm{t}, J=6.0 \mathrm{~Hz}, 2 \mathrm{H}), 3.40(\mathrm{t}, J=8.4 \mathrm{~Hz}, 2 \mathrm{H})$, $2.75(\mathrm{t}, J=6.8 \mathrm{~Hz}, 2 \mathrm{H}), 2.32$ (quint, $J=6.4 \mathrm{~Hz}, 2 \mathrm{H}) \mathrm{ppm} .{ }^{13} \mathrm{C}$ NMR (90.55 MHz, $\mathrm{CDCl}_{3}$ ): $\delta=170.7$ (C), 143.5 (C), 139.6 (C), $139.3(\mathrm{C}), 138.1(\mathrm{C}), 123.2(\mathrm{CH}), 120.0(\mathrm{CH}), 50.0\left(\mathrm{CH}_{2}\right), 44.2$ $\left(\mathrm{CH}_{2}\right), 32.1\left(\mathrm{CH}_{2}\right), 28.6\left(\mathrm{CH}_{2}\right), 27.3\left(\mathrm{CH}_{2}\right) \mathrm{ppm}$. IR $\left(\mathrm{CHCl}_{3}\right): \tilde{v}=$ $3022,1694,1609,1548,1438,1391,1344,1280 \mathrm{~cm}^{-1}$. UV/Vis (48 $\mu \mathrm{M}$ soln in MeCN): $\lambda\left(\varepsilon, \mathrm{M}^{-1} \mathrm{~cm}^{-1}\right)=202(17708), 225$ (11250), $350(8542) \mathrm{nm}$. MS: $m / z(\%)=313(1)[\mathrm{M}]^{+}, 209(100), 179(10)$,
163 (9), 117 (16), 107 (19), 105 (60), 77 (36). HRMS: calcd. for $\mathrm{C}_{12} \mathrm{H}_{12} \mathrm{~N}_{3} \mathrm{O}_{5}$ 313.0466; found 313.0480.

1-(3-Methoxycarbonylpropionyl)-5,7-dinitroindoline (1f): Yellow crystals, purified from remaining 5,7-dinitroindoline by recrystallisation (toluene/ethanol, 1:1) (m.p. $\quad 126-130{ }^{\circ} \mathrm{C}$ ). ${ }^{1} \mathrm{H} \quad \mathrm{NMR}$ $\left(360 \mathrm{MHz}, \mathrm{CDCl}_{3}\right): \delta=8.53(\mathrm{~s}, 1 \mathrm{H}), 8.25(\mathrm{~s}, 1 \mathrm{H}), 4.49$ (t, $J=$ $8.2 \mathrm{~Hz}, 2 \mathrm{H}), 3.68(\mathrm{~s}, 3 \mathrm{H}), 3.40(\mathrm{t}, J=8.2 \mathrm{~Hz}, 2 \mathrm{H}), 2.84-2.74(\mathrm{~m}$, $4 \mathrm{H}) \mathrm{ppm} .{ }^{13} \mathrm{C} \mathrm{NMR}\left(90.55 \mathrm{MHz}, \mathrm{CDCl}_{3}\right): \delta=172.9$ (C), 170.6 (C), 143.3 (C), 139.6 (C), 139.1 (C), 138.4 (C), $123.1(\mathrm{CH}), 119.8$ $(\mathrm{CH}), 52.0\left(\mathrm{CH}_{3}\right), 50.1\left(\mathrm{CH}_{2}\right), 30.2\left(\mathrm{CH}_{2}\right), 28.7\left(\mathrm{CH}_{2}\right), 28.5\left(\mathrm{CH}_{2}\right)$ ppm. IR $\left(\mathrm{CHCl}_{3}\right): \tilde{v}=3027,1733,1697,1612,1548,1439,1370$, 1344, 1283, $1162 \mathrm{~cm}^{-1}$. MS: $m / z(\%)=323(1)[\mathrm{M}]^{+}, 292(8), 209$ (12), 163 (3), 115 (100), 87 (14), 59 (24), 55 (57). HRMS: calcd. for $\mathrm{C}_{13} \mathrm{H}_{13} \mathrm{~N}_{3} \mathrm{O}_{7}$ 323.0754; found 323.0744.

Typical Procedure for the Photoacylation of Alcohols to Esters: All experiments were performed in anhydrous acetonitrile (dried by passing it, under an argon atmosphere, through a Grubbs purification system). ${ }^{[1]}$ A mixture of 1-acyl-5,7-dinitroindoline (25.1 mg, $0.1 \mathrm{mmol})$, the alcohol $(0.1 \mathrm{mmol}, 1$ equiv. $)$ in dry $\mathrm{MeCN}(1 \mathrm{~mL})$ was irradiated at $375 \mathrm{~nm}$ in a quartz tube for $16 \mathrm{~h}$, under an argon atmosphere, with vigorous stirring. The reaction mixture was then concentrated under reduced pressure, and the yield of the ester was then estimated by ${ }^{1} \mathrm{H}$ NMR spectroscopy. 5,7-Dinitroindoline is insoluble in hexane and was removed by trituration and filtration, unless specified otherwise. Evaporation of the hexane filtrate gave the ester and (when applicable) some unreacted alcohol.

Undecen-10-enyl Acetate (4a): Purification by flash column chromatography $\left[\mathrm{SiO}_{2}\right.$, hexane/EtOAc (9:1)]. ${ }^{1} \mathrm{H}$ NMR $(360 \mathrm{MHz}$, $\left.\mathrm{CDCl}_{3}\right): \delta=5.87-5.75(\mathrm{~m}, 1 \mathrm{H}), 5.01-4.91(\mathrm{~m}, 2 \mathrm{H}), 4.05(\mathrm{t}, J=$ $6.8 \mathrm{~Hz}, 2 \mathrm{H}), 2.06-2.01(\mathrm{~m}, 5 \mathrm{H}), 1.64$ (quint, $J=6.8 \mathrm{~Hz}, 2 \mathrm{H}$ ), $1.39-1.28(\mathrm{~m}, 12 \mathrm{H}) \mathrm{ppm} .{ }^{13} \mathrm{C} \mathrm{NMR}\left(90.55 \mathrm{MHz}, \mathrm{CDCl}_{3}\right): \delta=$ $171.4(\mathrm{C}), 139.4(\mathrm{CH}), 114.3(\mathrm{CH}), 64.8\left(\mathrm{CH}_{2}\right) 34.0\left(\mathrm{CH}_{2}\right), 29.6$ $\left(\mathrm{CH}_{2}\right), 29.5\left(\mathrm{CH}_{2}\right), 29.4\left(\mathrm{CH}_{2}\right), 29.2\left(\mathrm{CH}_{2}\right), 29.0\left(\mathrm{CH}_{2}\right), 28.7\left(\mathrm{CH}_{2}\right)$, $26.0\left(\mathrm{CH}_{2}\right), 21.2\left(\mathrm{CH}_{3}\right)$ ppm. IR (neat): $\tilde{v}=3078,2928,2856,1743$, 1641, 1464, 1441, 1388, 1366, 1240, 1039, 995, $910 \mathrm{~cm}^{-1}$. MS (ESI): $m / z(\%)=235.2(100)[\mathrm{M}+\mathrm{Na}]^{+}$. HRMS: calcd for $\mathrm{C}_{13} \mathrm{H}_{24} \mathrm{O}_{2}$ 212.1776; found 212.1772 .

9-Fluorenylmethyl Acetate (4b): Purification by flash column chromatography $\left[\mathrm{SiO}_{2}\right.$, hexane/EtOAc (9:1)]. ${ }^{1} \mathrm{H}$ NMR $(360 \mathrm{MHz}$, $\left.\mathrm{CDCl}_{3}\right): \delta=7.78(\mathrm{~d}, J=7.2 \mathrm{~Hz}, 2 \mathrm{H}), 7.61(\mathrm{~d}, J=7.2 \mathrm{~Hz}, 2 \mathrm{H})$, $7.42(\mathrm{dd}, J=7.2 \mathrm{~Hz}, 2 \mathrm{H}), 7.33(\mathrm{dd}, J=7.2 \mathrm{~Hz}, 2 \mathrm{H}), 4.37$ (d, $J=$ $7.2 \mathrm{~Hz}, 2 \mathrm{H}), 4.22(\mathrm{t}, J=7.3 \mathrm{~Hz}, 1 \mathrm{H}), 2.16(\mathrm{~s}, 3 \mathrm{H}) \mathrm{ppm} .{ }^{13} \mathrm{C}$ NMR (90.55 MHz, $\left.\mathrm{CDCl}_{3}\right): \delta=171.2(\mathrm{C}), 143.9$ (C), $141.4(\mathrm{C})$, $127.9(\mathrm{CH}), 127.2(\mathrm{CH}), 125.2(\mathrm{CH}), 120.2(\mathrm{CH}), 66.6\left(\mathrm{CH}_{2}\right), 46.9$ $(\mathrm{CH}), 21.2\left(\mathrm{CH}_{3}\right) \mathrm{ppm}$. IR $\left(\mathrm{CHCl}_{3}\right): \tilde{v}=3067,3041,3021,2951$, $1741,1449,1382,1363,1245,1036,759,740 \mathrm{~cm}^{-1}$. MS (ESI): $\mathrm{m} / z$ $(\%)=261.1(100)[\mathrm{M}+\mathrm{Na}]^{+}$. HRMS: calcd. for $\mathrm{C}_{16} \mathrm{H}_{14} \mathrm{O}_{2}$ 238.0994; found 238.0992.

Benzyl Acetate (4c): Purification by flash column chromatography $\left[\mathrm{SiO}_{2}\right.$, hexane/EtOAc (3:1)]. ${ }^{1} \mathrm{H}$ NMR $\left(360 \mathrm{MHz}, \mathrm{CDCl}_{3}\right): \delta=$ 7.40-7.30 (m, $5 \mathrm{H}), 5.11(\mathrm{~s}, 2 \mathrm{H}), 2.11(\mathrm{~s}, 3 \mathrm{H}) \mathrm{ppm} .{ }^{13} \mathrm{C} \mathrm{NMR}$ $\left(90.55 \mathrm{MHz}, \mathrm{CDCl}_{3}\right): \delta=171.1(\mathrm{C}), 136.1(\mathrm{C}), 128.7(2 \times \mathrm{CH})$, $-128.4(3 \times \mathrm{CH}), 66.5\left(\mathrm{CH}_{2}\right), 21.2\left(\mathrm{CH}_{3}\right) \mathrm{ppm}$. IR (neat): $\tilde{v}=3066$, 3035, 2956, 1743, 1498, 1456, 1381, 1363, 1233, 1028, 966, 750, $699 \mathrm{~cm}^{-1}$. MS (ESI): $\mathrm{m} / \mathrm{z}(\%)=173.0(100)[\mathrm{M}+\mathrm{Na}]^{+}$. HRMS: calcd. for $\mathrm{C}_{9} \mathrm{H}_{10} \mathrm{O}_{2}$ 150.0681; found 150.0673 .

3,4,5-Trimethoxybenzyl Acetate (4d): Purification by flash column chromatography $\left[\mathrm{SiO}_{2}\right.$, hexane/EtOAc (3:1)]. ${ }^{1} \mathrm{H}$ NMR $(360 \mathrm{MHz}$, $\left.\mathrm{CDCl}_{3}\right): \delta=6.59(\mathrm{~s}, 2 \mathrm{H}), 5.03(\mathrm{~s}, 2 \mathrm{H}), 3.87(\mathrm{~s}, 6 \mathrm{H}), 3.84(\mathrm{~s}, 3 \mathrm{H})$, $2.11(\mathrm{~s}, 3 \mathrm{H}) \mathrm{ppm} .{ }^{13} \mathrm{C}$ NMR $\left(90.55 \mathrm{MHz}, \mathrm{CDCl}_{3}\right): \delta=171.1(\mathrm{C})$, $153.5(2 \times \mathrm{C}), 138.1(\mathrm{C}), 131.6(\mathrm{C}), 105.7(2 \times \mathrm{CH}), 66.8\left(\mathrm{CH}_{2}\right)$, 
$61.0\left(\mathrm{CH}_{3}\right), 56.3\left(2 \times \mathrm{CH}_{3}\right), 21.2\left(\mathrm{CH}_{3}\right) \mathrm{ppm} . \mathrm{IR}$ (neat): $\tilde{v}=2999$, 2943, 1740, 1593, 1508, 1463, 1424, 1364, 1332, 1237, 1129, $1010 \mathrm{~cm}^{-1}$. MS (ESI): $\mathrm{m} / z(\%)=263.1(100)[\mathrm{M}+\mathrm{Na}]^{+}$. HRMS: calcd. for $\mathrm{C}_{12} \mathrm{H}_{16} \mathrm{O}_{5}$ 240.0998; found 240.0999.

(-)-Menthyl Acetate (4e): Purification by flash column chromatography $\left[\mathrm{SiO}_{2}\right.$, hexane/EtOAc (9:1)]. ${ }^{1} \mathrm{H}$ NMR $\left(360 \mathrm{MHz}, \mathrm{CDCl}_{3}\right): \delta$ $=4.66(\mathrm{td}, J=10.9,4.1 \mathrm{~Hz}, 1 \mathrm{H}), 2.03(\mathrm{~s}, 3 \mathrm{H}), 2.00-1.95(\mathrm{~m}, 1$ $\mathrm{H}), 1.92-1.79(\mathrm{~m}, 1 \mathrm{H}), 1.71-1.63(\mathrm{~m}, 2 \mathrm{H}), 1.55-1.41(\mathrm{~m}, 1 \mathrm{H})$, $1.39-1.31(\mathrm{~m}, 1 \mathrm{H}), 1.11-0.94(\mathrm{~m}, 2 \mathrm{H}), 0.9-0.88(\mathrm{~m}, 7 \mathrm{H}), 0.77-$ $0.75(\mathrm{~d}, 3 \mathrm{H}) \mathrm{ppm} .{ }^{13} \mathrm{C} \mathrm{NMR}\left(90.55 \mathrm{MHz}, \mathrm{CDCl}_{3}\right): \delta=170.8(\mathrm{C})$, $74.2(\mathrm{CH}), 47.0(\mathrm{CH}), 40.9\left(\mathrm{CH}_{2}\right), 34.2\left(\mathrm{CH}_{2}\right), 31.4(\mathrm{CH}), 26.3$ $(\mathrm{CH}), 23.5\left(\mathrm{CH}_{2}\right), 22.0\left(\mathrm{CH}_{3}\right), 21.3\left(\mathrm{CH}_{3}\right), 20.7\left(\mathrm{CH}_{3}\right), 16.4\left(\mathrm{CH}_{3}\right)$ ppm. IR (neat): $\tilde{v}=2957,2932,2871,1737,1457,1371,1246$, $1025 \mathrm{~cm}^{-1}$. MS (ESI): $\mathrm{m} / z(\%)=221.1(100)[\mathrm{M}+\mathrm{Na}]^{+}$. HRMS: calcd. for $\mathrm{C}_{12} \mathrm{H}_{22} \mathrm{O}_{2}$ 198.1620; found 198.1614.

2-Oxo-1,2-diphenylethyl Acetate (4f): The crude product was triturated with EtOAc. Evaporation of EtOAc gave the ester, the remaining alcohol and 5,7-dinitroindoline. Purification by flash column chromatography $\left[\mathrm{SiO}_{2}\right.$, hexane/EtOAc (6:1)]. ${ }^{1} \mathrm{H}$ NMR $\left(360 \mathrm{MHz}, \mathrm{CDCl}_{3}\right): \delta=7.93(\mathrm{~d}, J=7.2 \mathrm{~Hz}, 2 \mathrm{H}), 7.53-7.32(\mathrm{~m}, 8$ $\mathrm{H}), 6.86(\mathrm{~s}, 1 \mathrm{H}), 2.21(\mathrm{~s}, 3 \mathrm{H}) \mathrm{ppm} .{ }^{13} \mathrm{C} \mathrm{NMR}(90.55 \mathrm{MHz}$, $\left.\mathrm{CDCl}_{3}\right): \delta=193.7(\mathrm{C}), 170.5(\mathrm{C}), 134.6(\mathrm{C}), 133.6(\mathrm{C}), 133.5(\mathrm{CH})$, $129.3(\mathrm{CH}), 129.1(2 \times \mathrm{CH}), 128.8(2 \times \mathrm{CH}), 128.7(2 \times \mathrm{CH}), 128.6$ $(2 \times \mathrm{CH}), 77.6(\mathrm{CH}), 20.8\left(\mathrm{CH}_{3}\right) \mathrm{ppm} . \mathrm{IR}$ (neat): $\tilde{v}=1740,1697$, 1598, 1451, 1374, 1231, 1182, 1056, 1005, 973, 932, 758, 699, $526 \mathrm{~cm}^{-1}$. MS (ESI): $\mathrm{m} / z(\%)=277.1(100)[\mathrm{M}+\mathrm{Na}]^{+}$. HRMS: calcd for $\mathrm{C}_{16} \mathrm{H}_{14} \mathrm{O}_{3}$ 254.0943; found 254.0942.

1-Formylnaphthalen-2-yl Acetate (4 g): The crude product was triturated with EtOAc. Evaporation of EtOAc gave the ester, the remaining alcohol and 5,7-dinitroindoline. Purification by flash column chromatography $\left[\mathrm{SiO}_{2}\right.$, hexane/EtOAc (6:1)]. ${ }^{1} \mathrm{H}$ NMR $\left(360 \mathrm{MHz}, \mathrm{CDCl}_{3}\right): \delta=10.71(\mathrm{~s}, 1 \mathrm{H}), 9.14(\mathrm{~d}, J=8.6 \mathrm{~Hz}, 1 \mathrm{H})$, $8.10(\mathrm{~d}, J=9.1 \mathrm{~Hz}, 1 \mathrm{H}), 7.88(\mathrm{~d}, J=8.2 \mathrm{~Hz}, 1 \mathrm{H}), 7.68(\mathrm{t}, J=$ $7.3 \mathrm{~Hz}, 1 \mathrm{H}), 7.56(\mathrm{t}, J=7.5 \mathrm{~Hz}, 1 \mathrm{H}), 7.28(\mathrm{~d}, J=9.6 \mathrm{~Hz}, 1 \mathrm{H})$, $2.44(\mathrm{~s}, 3 \mathrm{H}) \mathrm{ppm} .{ }^{13} \mathrm{C} \mathrm{NMR}\left(90.55 \mathrm{MHz}, \mathrm{CDCl}_{3}\right): \delta=190.0(\mathrm{C})$, $169.2(\mathrm{C}), 154.4(\mathrm{C}), 136.5(\mathrm{CH}), 131.8(\mathrm{C}), 131.1(\mathrm{C}), 129.6(\mathrm{CH})$, $128.5(\mathrm{CH}), 126.7(\mathrm{CH}), 125.0(\mathrm{CH}), 121.6(\mathrm{CH}), 121.4(\mathrm{C}), 20.9$ $\left(\mathrm{CH}_{3}\right)$ ppm. IR $(\mathrm{KBr}): \tilde{v}=1767,1688,1617,1576,1511,1434$, 1372, 1342, 1189, 1161, 1061, 1036, 1017, 979, 893, 857, 826, 763, 743, 706, 675, $510 \mathrm{~cm}^{-1}$. MS (ESI): $m / z(\%)=237.0(100)[\mathrm{M}+$ $\mathrm{Na}]^{+}$. HRMS: calcd for $\mathrm{C}_{13} \mathrm{H}_{10} \mathrm{O}_{3}$ 214.0630; found 214.0625.

Cyclohexyl Acetate (4h): Purification by flash column chromatography $\left[\mathrm{SiO}_{2}\right.$, hexane/EtOAc (9:1)]. ${ }^{1} \mathrm{H}$ NMR $\left(360 \mathrm{MHz}, \mathrm{CDCl}_{3}\right): \delta$ $=4.76-4.70(\mathrm{~m}, 1 \mathrm{H}), 2.03(\mathrm{~s}, 3 \mathrm{H}), 1.87-1.82(\mathrm{~m}, 2 \mathrm{H}), 1.74-1.70$ $(\mathrm{m}, 2 \mathrm{H}) 1.57-1.52(\mathrm{~m}, 1 \mathrm{H}), 1.44-1.20(\mathrm{~m}, 5 \mathrm{H}) \mathrm{ppm} .{ }^{13} \mathrm{C} \mathrm{NMR}$ $\left(90.55 \mathrm{MHz}, \mathrm{CDCl}_{3}\right): \delta=170.6(\mathrm{C}), 72.7(\mathrm{CH}), 31.6\left(2 \times \mathrm{CH}_{2}\right)$, $25.4\left(\mathrm{CH}_{2}\right), 23.8\left(2 \times \mathrm{CH}_{2}\right), 21.5\left(\mathrm{CH}_{3}\right) \mathrm{ppm}$. IR (neat): $\tilde{v}=2940$, 2861, 1737, 1452, 1379, 1364, 1241, 1126, 1046, 1023, 968, 907, $735 \mathrm{~cm}^{-1}$. MS (ESI): $m / z(\%)=286.3\left\{2 \times[\mathrm{M}+\mathrm{H}]^{+}\right\}, 165.1(40)$ $[\mathrm{M}+\mathrm{Na}]^{+}$.

trans-(-)-Myrtyl Acetate (4i): Purification by flash column chromatography $\left[\mathrm{SiO}_{2}\right.$, hexane/EtOAc (9:1)]. ${ }^{1} \mathrm{H}$ NMR $(360 \mathrm{MHz}$, $\left.\mathrm{CDCl}_{3}\right): \delta=3.92-3.81(\mathrm{~m}, 2 \mathrm{H}), 2.34-2.25(\mathrm{~m}, 1 \mathrm{H}), 2.08-2.02(\mathrm{~m}$, $1 \mathrm{H}), 2.03(\mathrm{~s}, 3 \mathrm{H}), 1.89-1.71(\mathrm{~m}, 4 \mathrm{H}), 1.67-1.59(\mathrm{~m}, 1 \mathrm{H}), 1.34$ $1.18(\mathrm{~m}, 2 \mathrm{H}), 1.21(\mathrm{~s}, 3 \mathrm{H}), 0.84(\mathrm{~s}, 3 \mathrm{H}) \mathrm{ppm} .{ }^{13} \mathrm{C} \mathrm{NMR}$ $\left(90.55 \mathrm{MHz}, \mathrm{CDCl}_{3}\right) \delta=171.4(\mathrm{C}), 68.0\left(\mathrm{CH}_{2}\right), 42.3(\mathrm{CH}), 40.8$ $(\mathrm{CH}), 39.1(\mathrm{C}), 34.1(\mathrm{CH}), 26.6\left(\mathrm{CH}_{2}\right), 23.9\left(\mathrm{CH}_{3}\right), 23.3\left(\mathrm{CH}_{2}\right)$, $21.0\left(\mathrm{CH}_{3}\right), 20.1\left(\mathrm{CH}_{3}\right), 18.1\left(\mathrm{CH}_{2}\right) \mathrm{ppm}$. IR (neat): $\tilde{v}=2979,2945$, 2917, 2870, 1743, 1463, 1386, 1367, 1236, 1031, $980 \mathrm{~cm}^{-1}$. MS (ESI): $m / z(\%)=219.1(100)[\mathrm{M}+\mathrm{Na}]^{+}$. HRMS: calcd. for $\mathrm{C}_{12} \mathrm{H}_{20} \mathrm{O}_{2}$ 196.1463; found 196.1456.
(士)-Linaloyl Acetate (4j): Purification by flash column chromatography $\left[\mathrm{SiO}_{2}\right.$, hexane/EtOAc (9:1)]. ${ }^{1} \mathrm{H}$ NMR $\left(360 \mathrm{MHz}, \mathrm{CDCl}_{3}\right): \delta$ $=6.01-5.93(\mathrm{~m}, 1 \mathrm{H}), 5.17,5.07(\mathrm{~m}, 3 \mathrm{H}), 2.01(\mathrm{~s}, 3 \mathrm{H}), 1.96(\mathrm{t}, J$ $=7.5 \mathrm{~Hz}, 2 \mathrm{H}), 1.89-1.71(\mathrm{~m}, 2 \mathrm{H}), 1.67(\mathrm{~s}, 3 \mathrm{H}), 1.59(\mathrm{~s}, 3 \mathrm{H}), 1.53$ (s, $3 \mathrm{H}) \mathrm{ppm} .{ }^{13} \mathrm{C}$ NMR $\left(90.55 \mathrm{MHz}, \mathrm{CDCl}_{3}\right) \delta=170.0(\mathrm{C}), 141.8$ $(\mathrm{CH}), 131.8(\mathrm{C}), 123.8(\mathrm{CH}), 113.1\left(\mathrm{CH}_{2}\right), 82.9(\mathrm{C}), 39.7\left(\mathrm{CH}_{2}\right)$, $25.7\left(\mathrm{CH}_{3}\right), 23.6\left(\mathrm{CH}_{3}\right), 22.3\left(\mathrm{CH}_{2}\right), 22.2\left(\mathrm{CH}_{3}\right), 17.6\left(\mathrm{CH}_{3}\right) \mathrm{ppm}$. IR (neat): $\tilde{v}=2973,2930,2861,1740,1450,1370,1248,1173,1093$, 1019, $922 \mathrm{~cm}^{-1}$. HRMS: calcd. for $\mathrm{C}_{12} \mathrm{H}_{20} \mathrm{O}_{2}$ 196.1463; found 196.1458.

Geranyl Acetate (4k): Purification by flash column chromatography $\left[\mathrm{SiO}_{2}\right.$, hexane/EtOAc (9:1)]. ${ }^{1} \mathrm{H}$ NMR $\left(360 \mathrm{MHz}, \mathrm{CDCl}_{3}\right): \delta=5.34$ $(\mathrm{t}, J=7.2 \mathrm{~Hz}, 1 \mathrm{H}), 5.08(\mathrm{t}, J=6.8 \mathrm{~Hz}, 1 \mathrm{H}), 4.58(\mathrm{~d}, J=7.3 \mathrm{~Hz}$, $2 \mathrm{H}), 2.14-2.00(\mathrm{~m}, 4 \mathrm{H}), 2.05(\mathrm{~s}, 3 \mathrm{H}), 1.70(\mathrm{~s}, 3 \mathrm{H}), 1.68(\mathrm{~s}, 3 \mathrm{H})$, $1.60(\mathrm{~s}, 3 \mathrm{H}) \mathrm{ppm} .{ }^{13} \mathrm{C} \mathrm{NMR}\left(90.55 \mathrm{MHz}, \mathrm{CDCl}_{3}\right): \delta=171.2(\mathrm{C})$, $142.3(\mathrm{C}), 131.8(\mathrm{C}), 123.7(\mathrm{CH}), 118.2(\mathrm{CH}), 61.4\left(\mathrm{CH}_{2}\right), 39.5$ $\left(\mathrm{CH}_{2}\right), 26.3\left(\mathrm{CH}_{2}\right), 25.7\left(\mathrm{CH}_{3}\right), 21.1\left(\mathrm{CH}_{3}\right), 17.7\left(\mathrm{CH}_{3}\right), 16.5\left(\mathrm{CH}_{3}\right)$ ppm. IR (neat): $\tilde{v}=2969,2926,2859,1742,1446,1380,1369,1233$, 1024, 982, $955 \mathrm{~cm}^{-1}$. MS (ESI): $\mathrm{m} / z(\%)=219.1(100)[\mathrm{M}+\mathrm{Na}]^{+}$. HRMS: calcd. for $\mathrm{C}_{12} \mathrm{H}_{20} \mathrm{O}_{2}$ 196.1463; found 196.1457.

1-Ethynylcyclohexyl Acetate (4l): Purification by flash column chromatography $\left[\mathrm{SiO}_{2}\right.$, hexane/EtOAc (9:1)]. ${ }^{1} \mathrm{H}$ NMR $(360 \mathrm{MHz}$, $\left.\mathrm{CDCl}_{3}\right): \delta=2.60(\mathrm{~s}, 1 \mathrm{H}), 2.16-2.10(\mathrm{~m}, 2 \mathrm{H}), 2.05(\mathrm{~s}, 3 \mathrm{H}), 1.88-$ $1.81(\mathrm{~m}, 2 \mathrm{H}), 1.65-1.59(\mathrm{~m}, 4 \mathrm{H}), 1.55-1.48(\mathrm{~m}, 1 \mathrm{H}), 1.38-1.27$ (m, $1 \mathrm{H})$ ppm. ${ }^{13} \mathrm{C}$ NMR $\left(90.55 \mathrm{MHz}, \mathrm{CDCl}_{3}\right): \delta=169.3(\mathrm{C}), 83.7$ (C), $75.1(\mathrm{C}), 74.2(\mathrm{CH}), 36.9\left(2 \times \mathrm{H}_{2}\right), 25.1\left(\mathrm{CH}_{2}\right), 22.4\left(2 \times \mathrm{CH}_{2}\right)$, $21.9\left(\mathrm{CH}_{3}\right)$ ppm. IR (neat): $\tilde{v}=3283,2934,2863,2113,1745,1450$, 1368, 1231, 1145, 1025, 957, $663 \mathrm{~cm}^{-1}$. GC-MS: $m / z(\%)=166.1$ (2) $[\mathrm{M}]^{+}, 124.0$ (58), 109 (40), 106 (50), 95 (74), 91 (10), 81 (92), 79 (68), 67 (52). HRMS: calcd. for $\mathrm{C}_{10} \mathrm{H}_{14} \mathrm{O}_{2}$ 166.0994; found 160.0983

$\mathbf{1 , 2 , 3 , 4 , 6 - P e n t a - O}$-acetyl- $\boldsymbol{\beta}$-D-glucose $(\mathbf{4 m})$ : The crude product was triturated with EtOAc. Evaporation of EtOAc gave the ester, the remaining alcohol and 5,7-dinitroindoline. Purification by flash column chromatography $\left(\mathrm{SiO}_{2}, \mathrm{CH}_{2} \mathrm{Cl}_{2}\right)$ to remove 5,7-dinitroindoline and $\left[\mathrm{SiO}_{2}\right.$, hexane/EtOAc (1:1)]. ${ }^{1} \mathrm{H}$ NMR $\left(360 \mathrm{MHz}, \mathrm{CDCl}_{3}\right)$ : $\delta=5.71(\mathrm{~d}, J=8.2 \mathrm{~Hz}, 1 \mathrm{H}), 5.25(\mathrm{dd}, J=9.3 \mathrm{~Hz}, 1 \mathrm{H}), 5.15-5.10$ $(\mathrm{m}, 2 \mathrm{H}), 4.29$ (dd, $J=12.5,4.3 \mathrm{~Hz}, 1 \mathrm{H}), 4.11$ (dd, $J=12.5$, $2.1 \mathrm{~Hz}, 1 \mathrm{H}), 3.84(\mathrm{dm}, J=10 \mathrm{~Hz}, 1 \mathrm{H}), 2.11(\mathrm{~s}, 3 \mathrm{H}), 2.08(\mathrm{~s}, 3 \mathrm{H})$, $2.03(\mathrm{~s}, 6 \mathrm{H}), 2.01(\mathrm{~s}, 3 \mathrm{H}) \mathrm{ppm} .{ }^{13} \mathrm{C}$ NMR $\left(90.55 \mathrm{MHz}, \mathrm{CDCl}_{3}\right): \delta$ $=170.6(\mathrm{C}), 170.1(\mathrm{C}), 169.4(\mathrm{C}), 169.2(\mathrm{C}), 169.0(\mathrm{C}), 91.7(\mathrm{CH})$, $72.7(2 \times \mathrm{CH}), 70.2(\mathrm{CH}), 67.7(\mathrm{CH}), 61.4\left(\mathrm{CH}_{2}\right), 20.8\left(\mathrm{CH}_{3}\right), 20.7$ $\left(\mathrm{CH}_{3}\right), 20.6\left(3 \times \mathrm{CH}_{3}\right) \mathrm{ppm}$. IR (neat/ $\left.\mathrm{CHCl}_{3}\right): \tilde{v}=3025,1760,1370$, 1222, 1080, 1040, 758, $669 \mathrm{~cm}^{-1}$. HRMS: calcd. for $\mathrm{C}_{16} \mathrm{H}_{22} \mathrm{O}_{11}$ 390.1162 ; found 390.1156 .

( \pm )-1-Phenylethyl Acetate (4n): Purification by flash column chromatography $\left[\mathrm{SiO}_{2}\right.$, hexane/EtOAc (9:1)]. ${ }^{1} \mathrm{H}$ NMR $(360 \mathrm{MHz}$, $\left.\mathrm{CDCl}_{3}\right): \delta=7.36-7.28(\mathrm{~m}, 5 \mathrm{H}), 5.88(\mathrm{q}, J=6.8 \mathrm{~Hz}, 1 \mathrm{H}), 2.08(\mathrm{~s}$, $3 \mathrm{H}), 1.54(\mathrm{~d}, J=6.8 \mathrm{~Hz}, 3 \mathrm{H}) \mathrm{ppm} .{ }^{13} \mathrm{C} \mathrm{NMR}(90.55 \mathrm{MHz}$, $\left.\mathrm{CDCl}_{3}\right): \delta=170.3(\mathrm{C}), 141.7(\mathrm{C}), 128.5(2 \times \mathrm{CH}), 127.9(\mathrm{CH}), 126.1$ $(2 \times \mathrm{CH}), 72.3(\mathrm{CH}), 22.2\left(\mathrm{CH}_{3}\right), 21.4\left(\mathrm{CH}_{3}\right) \mathrm{ppm}$. IR (neat): $\tilde{v}=$ 3065, 3034, 2983, 2936, 1744, 1495, 1453, 1372, 1242, 1210, 1065, 1027, 945, 761, 700, $539 \mathrm{~cm}^{-1}$. HRMS: calcd. for $\mathrm{C}_{10} \mathrm{H}_{12} \mathrm{O}_{2}$ 164.0837; found 164.0831 .

Cinnamyl Acetate (4o): Purification by flash column chromatography $\left[\mathrm{SiO}_{2}\right.$, hexane/EtOAc (9:1)]. ${ }^{1} \mathrm{H}$ NMR (360 MHz, $\left.\mathrm{CDCl}_{3}\right): \delta$ $=7.40(\mathrm{~d}, J=7.3 \mathrm{~Hz} ; 2 \mathrm{H}), 7.33(\mathrm{dd}, J=7.3 \mathrm{~Hz}, 2 \mathrm{H}), 7.26(\mathrm{dd}$, $J=7.1 \mathrm{~Hz}, 1 \mathrm{H}), 6.66(\mathrm{~d}, J=15.9 \mathrm{~Hz}, 1 \mathrm{H}), 6.29(\mathrm{dt}, J=15.9$, $6.6 \mathrm{~Hz}, 1 \mathrm{H}), 4.73(\mathrm{~d}, J=6.4 \mathrm{~Hz}, 2 \mathrm{H}), 2.10(\mathrm{~s}, 3 \mathrm{H}) \mathrm{ppm} .{ }^{13} \mathrm{C}$ NMR (90.55 MHz, $\left.\mathrm{CDCl}_{3}\right): \delta=170.9(\mathrm{C}), 136.2(\mathrm{C}), 134.2(\mathrm{CH})$, $128.6(2 \times \mathrm{CH}), 128.1(\mathrm{CH}), 126.6(2 \times \mathrm{CH}), 123.1(\mathrm{CH}), 65.1$ $\left(\mathrm{CH}_{2}\right), 21.0\left(\mathrm{CH}_{3}\right)$ ppm. IR (neat): $\tilde{v}=3060,3029,2945,1739$, 
1495, 1490, 1455, 1381, 1363, 1233, 1027, 967, 747, $694 \mathrm{~cm}^{-1}$. HRMS: calcd. for $\mathrm{C}_{11} \mathrm{H}_{12} \mathrm{O}_{2}$ 176.0837; found 176.0832.

3',5'-Dimethoxy-2-oxo-1,2-diphenyl Acetate (4p): For this photosensitive ester, the working wavelength was $405 \mathrm{~nm}$. The crude product was triturated with EtOAc and evaporation of EtOAc gave the ester, the remaining alcohol and 5,7-dinitroindoline. Purification by flash column chromatography $\left[\mathrm{SiO}_{2}\right.$, hexane/EtOAc (3:1)]. ${ }^{1} \mathrm{H}$ NMR $\left(360 \mathrm{MHz}, \mathrm{CDCl}_{3}\right): \delta=7.94(\mathrm{~d}, J=7.8 \mathrm{~Hz}, 2 \mathrm{H}), 7.52$ (t, $J=7.3 \mathrm{~Hz}, 1 \mathrm{H}), 7.41(\mathrm{t}, J=7.8 \mathrm{~Hz}, 2 \mathrm{H}), 6.75(\mathrm{~s}, 1 \mathrm{H}), 6.59$ $(\mathrm{d}, J=1.8 \mathrm{~Hz}, 2 \mathrm{H}), 6.42(\mathrm{t}, J=1.8 \mathrm{~Hz}, 1 \mathrm{H}), 3.76(\mathrm{~s}, 6 \mathrm{H}), 2.21$ (s, $3 \mathrm{H}) \mathrm{ppm} .{ }^{13} \mathrm{C} \mathrm{NMR}\left(90.55 \mathrm{MHz}, \mathrm{CDCl}_{3}\right): \delta=193.5$ (C), 170.4 (C), $161.2(2 \times \mathrm{C}), 135.5(\mathrm{C}), 134.5(\mathrm{C}), 133.5(\mathrm{CH}), 128.8(2 \times \mathrm{CH})$, $128.6(2 \times \mathrm{CH}), \quad 106.7(2 \times \mathrm{CH}), 101.2(\mathrm{CH}), 77.6(\mathrm{CH}), 55.4$ $\left(2 \times \mathrm{CH}_{3}\right), 20.8\left(\mathrm{CH}_{3}\right) \mathrm{ppm}$. IR (neat): $\tilde{\mathrm{v}}=3006,2942,2841,1745$, $1698,1598,1464,1431,1374,1355,1281,1231,1160,1056,1002$, 839, $691 \mathrm{~cm}^{-1}$. HRMS: calcd. for $\mathrm{C}_{18} \mathrm{H}_{18} \mathrm{O}_{5}$ 314.1154; found 314.1147

Undec-10-enyl Propionate (6b): Purification by flash column chromatography $\left[\mathrm{SiO}_{2}\right.$, hexane/EtOAc (9:1)]. ${ }^{1} \mathrm{H}$ NMR $(360 \mathrm{MHz}$, $\left.\mathrm{CDCl}_{3}\right): \delta=5.85-5.77(\mathrm{~m}, 1 \mathrm{H}), 5.01-4.91(\mathrm{~m}, 2 \mathrm{H}), 4.05(\mathrm{t}, J=$ $6.8 \mathrm{~Hz}, 2 \mathrm{H}), 2.32(\mathrm{q}, J=7.7 \mathrm{~Hz}, 2 \mathrm{H}), 2.06-2.00(\mathrm{~m}, 2 \mathrm{H}), 1.63-$ $1.58(\mathrm{~m}, 2 \mathrm{H}), 1.37-1.28(\mathrm{~m}, 12 \mathrm{H}), 1.14(\mathrm{t}, J=7.7 \mathrm{~Hz}, 3 \mathrm{H}) \mathrm{ppm}$. ${ }^{13} \mathrm{C}$ NMR (90.55 MHz, $\left.\mathrm{CDCl}_{3}\right): \delta=174.6(\mathrm{C}), 139.2(\mathrm{CH}), 114.3$ $\left(\mathrm{CH}_{2}\right), 64.5\left(\mathrm{CH}_{2}\right) 33.8\left(\mathrm{CH}_{2}\right), 29.4\left(\mathrm{CH}_{2}\right), 29.4\left(\mathrm{CH}_{2}\right), 29.2\left(\mathrm{CH}_{2}\right)$, $29.1\left(\mathrm{CH}_{2}\right), 28.9\left(\mathrm{CH}_{2}\right), 28.6\left(\mathrm{CH}_{2}\right), 27.6\left(\mathrm{CH}_{2}\right), 25.9\left(\mathrm{CH}_{2}\right), 9.2$ $\left(\mathrm{CH}_{3}\right)$ ppm. IR (neat): $\tilde{v}=2979,2929,2856,1741,1464,1350$, 1274, 1187, 1084, 995, $910 \mathrm{~cm}^{-1}$. HRMS: calcd. for $\mathrm{C}_{16} \mathrm{H}_{26} \mathrm{O}_{2}$ 226.1933; found 226.1929 .

Undec-10-enyl Lauroate (6c): Purification by flash column chromatography $\left[\mathrm{SiO}_{2}\right.$, hexane/EtOAc (9:1)]. ${ }^{1} \mathrm{H}$ NMR $(360 \mathrm{MHz}$, $\left.\mathrm{CDCl}_{3}\right): \delta=5.85-5.77(\mathrm{~m}, 1 \mathrm{H}), 5.01-4.91(\mathrm{~m}, 2 \mathrm{H}), 4.05(\mathrm{t}, J=$ $6.8 \mathrm{~Hz}, 2 \mathrm{H}), 2.28(\mathrm{t}, J=7.5 \mathrm{~Hz}, 2 \mathrm{H}), 2.06-2.01(\mathrm{~m}, 2 \mathrm{H}), 1.63-$ $1.57(\mathrm{~m}, 4 \mathrm{H}), 1.37-1.25(\mathrm{~m}, 28 \mathrm{H}), 0.88(\mathrm{t}, J=6.5 \mathrm{~Hz}, 3 \mathrm{H}) \mathrm{ppm}$. ${ }^{13} \mathrm{C}$ NMR (90.55 MHz, $\left.\mathrm{CDCl}_{3}\right): \delta=174.0(\mathrm{C}), 139.2(\mathrm{CH}), 114.1$ $\left(\mathrm{CH}_{2}\right), 64.4\left(\mathrm{CH}_{2}\right), 34.4\left(\mathrm{CH}_{2}\right), 33.8\left(\mathrm{CH}_{2}\right), 31.9\left(\mathrm{CH}_{2}\right), 29.6$ $\left(2 \times \mathrm{CH}_{2}\right), 29.5\left(\mathrm{CH}_{2}\right), 29.5\left(\mathrm{CH}_{2}\right), 29.4\left(\mathrm{CH}_{2}\right), 29.3\left(\mathrm{CH}_{2}\right), 29.3$ $\left(\mathrm{CH}_{2}\right), 29.2\left(\mathrm{CH}_{2}\right), 29.2\left(\mathrm{CH}_{2}\right), 29.1\left(\mathrm{CH}_{2}\right), 28.9\left(\mathrm{CH}_{2}\right), 28.6\left(\mathrm{CH}_{2}\right)$, $25.9\left(\mathrm{CH}_{2}\right), 25.0\left(\mathrm{CH}_{2}\right), 22.7\left(\mathrm{CH}_{2}\right), 14.1\left(\mathrm{CH}_{3}\right)$ ppm. IR (neat): $\tilde{v}$ $=2929,2856,1739,1467,1173,909 \mathrm{~cm}^{-1}$. HRMS: calcd. for $\mathrm{C}_{23} \mathrm{H}_{44} \mathrm{O}_{2}$ 352.3341; found 352.3334 .

Undec-10-enyl Benzoate (6d): Purification by flash column chromatography $\left[\mathrm{SiO}_{2}\right.$, hexane/EtOAc $\left.(9: 1)\right] .{ }^{1} \mathrm{H}$ NMR $(360 \mathrm{MHz}$, $\left.\mathrm{CDCl}_{3}\right): \delta=8.05(\mathrm{~d}, J=7.3 \mathrm{~Hz}, 2 \mathrm{H}), 7.55(\mathrm{dd}, J=7.7,7.3 \mathrm{~Hz} 1$ $\mathrm{H}), 7.44(\mathrm{dd}, J=8.2,7.3 \mathrm{~Hz} \mathrm{1} \mathrm{H}), 5.87-5.75(\mathrm{~m}, 1 \mathrm{H}), 5.01-4.92$ $(\mathrm{m}, 2 \mathrm{H}), 4.31(\mathrm{t}, J=6.8 \mathrm{~Hz}, 2 \mathrm{H}), 2.04(\mathrm{q}, J=7.2 \mathrm{~Hz}, 2 \mathrm{H}), 1.80$ $1.73(\mathrm{~m}, 2 \mathrm{H}), 1.46-1.30(\mathrm{~m}, 12 \mathrm{H}) \mathrm{ppm} .{ }^{13} \mathrm{C} \mathrm{NMR}(90.55 \mathrm{MHz}$, $\left.\mathrm{CDCl}_{3}\right): \delta=166.7(\mathrm{C}), 139.2(\mathrm{CH}), 132.8(\mathrm{CH}), 130.5(\mathrm{C}), 129.5$ $(2 \times \mathrm{CH}), 128.3(2 \times \mathrm{CH}), 114.1\left(\mathrm{CH}_{2}\right), 65.1\left(\mathrm{CH}_{2}\right) 33.8\left(\mathrm{CH}_{2}\right), 29.5$ $\left(\mathrm{CH}_{2}\right), 29.4\left(\mathrm{CH}_{2}\right), 29.2\left(\mathrm{CH}_{2}\right), 29.1\left(\mathrm{CH}_{2}\right), 28.9\left(\mathrm{CH}_{2}\right), 28.7\left(\mathrm{CH}_{2}\right)$, $26.0\left(\mathrm{CH}_{3}\right) \mathrm{ppm}$. IR (neat): $\tilde{\mathrm{v}}=2928,2855,1722,1453,1314,1274$, 1176, 1114, 1070, 1028, 910, $712 \mathrm{~cm}^{-1}$. HRMS: calcd. for $\mathrm{C}_{18} \mathrm{H}_{26} \mathrm{O}_{2}$ 274.1933; found 274.1929 .
Undec-10-enyl 4-Chlorobutanoate (6e): Purification by flash column chromatography $\left[\mathrm{SiO}_{2}\right.$, hexane/EtOAc (9:1)]. ${ }^{1} \mathrm{H}$ NMR (360 MHz, $\left.\mathrm{CDCl}_{3}\right): \delta=5.85-5.77(\mathrm{~m}, 1 \mathrm{H}), 5.02-4.92(\mathrm{~m}, 2 \mathrm{H}), 4.07(\mathrm{t}, J=$ $6.8 \mathrm{~Hz}, 2 \mathrm{H}), 3.60(\mathrm{t}, J=6.4 \mathrm{~Hz}, 2 \mathrm{H}), 2.50(\mathrm{t}, J=7.2 \mathrm{~Hz}, 2 \mathrm{H})$, 2.13-2.01 (m, $4 \mathrm{H}), 1.64-1.55(\mathrm{~m}, 2 \mathrm{H}), 1.42-1.29(\mathrm{~m}, 12 \mathrm{H}) \mathrm{ppm}$. ${ }^{13} \mathrm{C}$ NMR (90.55 MHz, $\left.\mathrm{CDCl}_{3}\right): \delta=172.8(\mathrm{C}), 139.2(\mathrm{CH}), 114.1$ $\left(\mathrm{CH}_{2}\right), 64.8\left(\mathrm{CH}_{2}\right) 44.1\left(\mathrm{CH}_{2}\right), 33.8\left(\mathrm{CH}_{2}\right), 31.2\left(\mathrm{CH}_{2}\right), 29.4$ $\left(2 \times \mathrm{CH}_{2}\right), 29.2\left(\mathrm{CH}_{2}\right), 29.1\left(\mathrm{CH}_{2}\right), 28.9\left(\mathrm{CH}_{2}\right), 28.6\left(\mathrm{CH}_{2}\right), 27.7$ $\left(\mathrm{CH}_{2}\right), 25.9\left(\mathrm{CH}_{2}\right)$ ppm. IR (neat): $\tilde{v}=2930,2856,1737,1466$, 1299, 1240, 1206, 1176, 1147, $911 \mathrm{~cm}^{-1}$. HRMS: calcd. for $\mathrm{C}_{18} \mathrm{H}_{26} \mathrm{O}_{2} 274.1700 ; 274.1697$.

Methyl Undec-10-enylsuccinate (6f): Purification by flash column chromatography $\left[\mathrm{SiO}_{2}\right.$, hexane/EtOAc (4:1)]. ${ }^{1} \mathrm{H}$ NMR $(360 \mathrm{MHz}$, $\left.\mathrm{CDCl}_{3}\right): \delta=5.85-5.75(\mathrm{~m}, 1 \mathrm{H}), 5.01-4.91(\mathrm{~m}, 2 \mathrm{H}), 4.08(\mathrm{t}, J=$ $6.8 \mathrm{~Hz}, 2 \mathrm{H}), 3.69$ (s, $3 \mathrm{H}), 2.63$ (br. s, $4 \mathrm{H}), 2.06-2.01$ (m, $2 \mathrm{H})$, $1.65-1.58(\mathrm{~m}, 2 \mathrm{H}), 1.39-1.28(\mathrm{~m}, 12 \mathrm{H})$ ppm. ${ }^{13} \mathrm{C} \mathrm{NMR}$ $\left(90.55 \mathrm{MHz}, \mathrm{CDCl}_{3}\right): \delta=172.8(\mathrm{C}), 172.4(\mathrm{C}), 139.2(\mathrm{CH}), 114.1$ $\left(\mathrm{CH}_{2}\right), 64.9\left(\mathrm{CH}_{2}\right), 51.8\left(\mathrm{CH}_{3}\right), 33.8\left(\mathrm{CH}_{2}\right), 29.4\left(\mathrm{CH}_{2}\right), 29.4\left(\mathrm{CH}_{2}\right)$, $29.2\left(\mathrm{CH}_{2}\right), 29.2\left(\mathrm{CH}_{2}\right), 29.1\left(\mathrm{CH}_{2}\right), 28.9\left(\mathrm{CH}_{2}\right), 28.9\left(\mathrm{CH}_{2}\right), 28.6$ $\left(\mathrm{CH}_{2}\right), 25.8\left(\mathrm{CH}_{2}\right) \mathrm{ppm}$. IR (neat): $\tilde{v}=2927,2856,1739,1438,1357$, 1214, 1162, $997 \mathrm{~cm}^{-1}$. HRMS: calcd. for $\mathrm{C}_{16} \mathrm{H}_{28} \mathrm{O}_{4} 284.1988$; found 284.1983.

Supporting Information (see footnote on the first page of this article): ${ }^{1} \mathrm{H}$ - and ${ }^{13} \mathrm{C}-\mathrm{NMR}$ spectra for $\mathbf{1 a}-\mathbf{f}, \mathbf{4 a}-\mathbf{p}$ and $\mathbf{6 b}-\mathbf{f}$.

\section{Acknowledgments}

We thank the Swiss National Science Foundation for their generous support (grant 620-066063).

[1] J. Otera, Chem. Rev. 1993, 93, 1449-1470 and references cited therein.

[2] A search in SciFinder with the keyword "esterification" in the CAPLUS database returned 126357 hits (October 2005).

[3] S. Pass, B. Amit, A. Patchornik, J. Am. Chem. Soc. 1981, 103, 7674-7675.

[4] K. C. Nicolaou, B. S. Safina, N. Winssinger, Synlett 2001, 900 903.

[5] C. Helgen, C. G. Bochet, Synlett 2001, 1968-1971.

[6] C. Helgen, C. G. Bochet, J. Org. Chem. 2003, 68, 2483-2486.

[7] B. Amit, D. A. Ben-Efraim, A. Patchornik, J. Am. Chem. Soc 1976, $98,843-844$

[8] A. D. Cohen, C. Helgen, C. G. Bochet, J. P. Toscano, Org. Lett 2005, 7, 2845-2847.

[9] For other recent mechanistic study, see: J. Morrison, P. Wan, J. E. T. Corrie, G. Papageorgiou, Photochem. Photobiol. Sci. 2002, 1, 960-969.

[10] a) A. Blanc, C. G. Bochet, J. Org. Chem. 2002, 67, 5567-5577; b) C. G. Bochet, Angew. Chem. Int. Ed. 2001, 40, 2071-2073.

[11] A. B. Pangborn, M. A. Giardello, R. H. Grubbs, R. K. Rosen, F. J. Timmers, Organometallics 1996, 15, 1518-1520. 


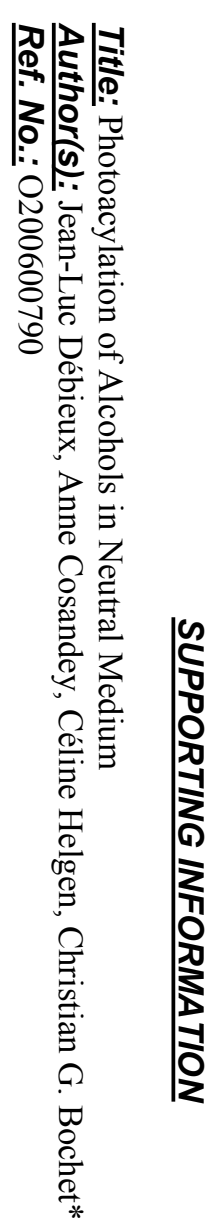


1-Propionyl-5,7-dinitroindoline

\begin{tabular}{|c|c|c|c|c|c|}
\hline Acquisition Time (sec) & 2.2021 & Com m ent $\quad \mathrm{N}$-propy & N-propyl, dinitroindoline PROTONNR CDCl3 u jld 2 & Date & 10 Mar 2006 11:58:56 \\
\hline File Nam e & \multicolumn{3}{|c|}{ IIHOMEIDebieuxJIMy DocumentsIChimielDoctorat|NMRIdinitropropyl_001001r } & Frequency $(\mathrm{M} \mathrm{H} z$ ) & 360.13 \\
\hline Nucleus & $1 \mathrm{H}$ & Num beroftransients 32 & $\mathrm{dp} \times 360$ & O riginalPoints Count & 16384 \\
\hline Owner & nmruser & Points Count & Pulse Sequence & ReceiverGain & 322.50 \\
\hline SW (cyclical) (H z) & 7440.48 & Solvent $\quad$ CHLOR & CHLOROFORM-d & Spectrum Offset $(H z)$ & 2210.5950 \\
\hline Sw eep W idth (H z) & 7440.02 & Tem perature (degree C) 27.000 & & & \\
\hline
\end{tabular}

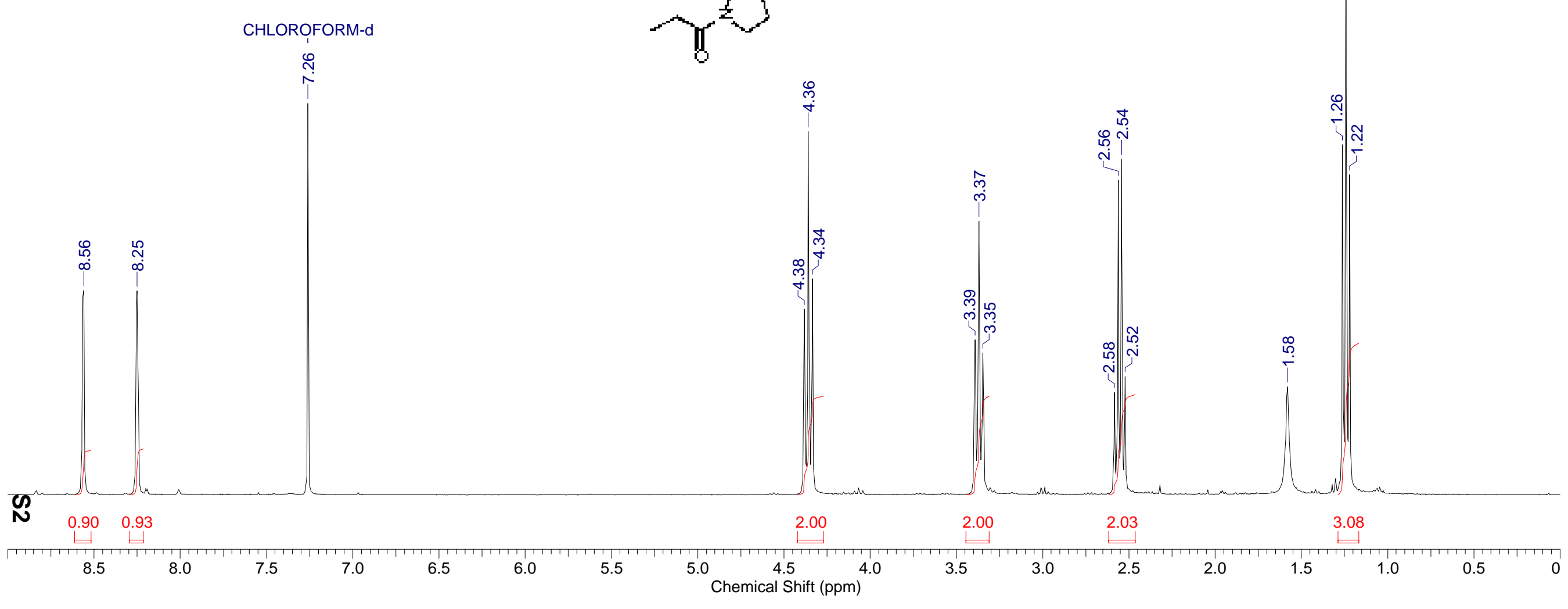




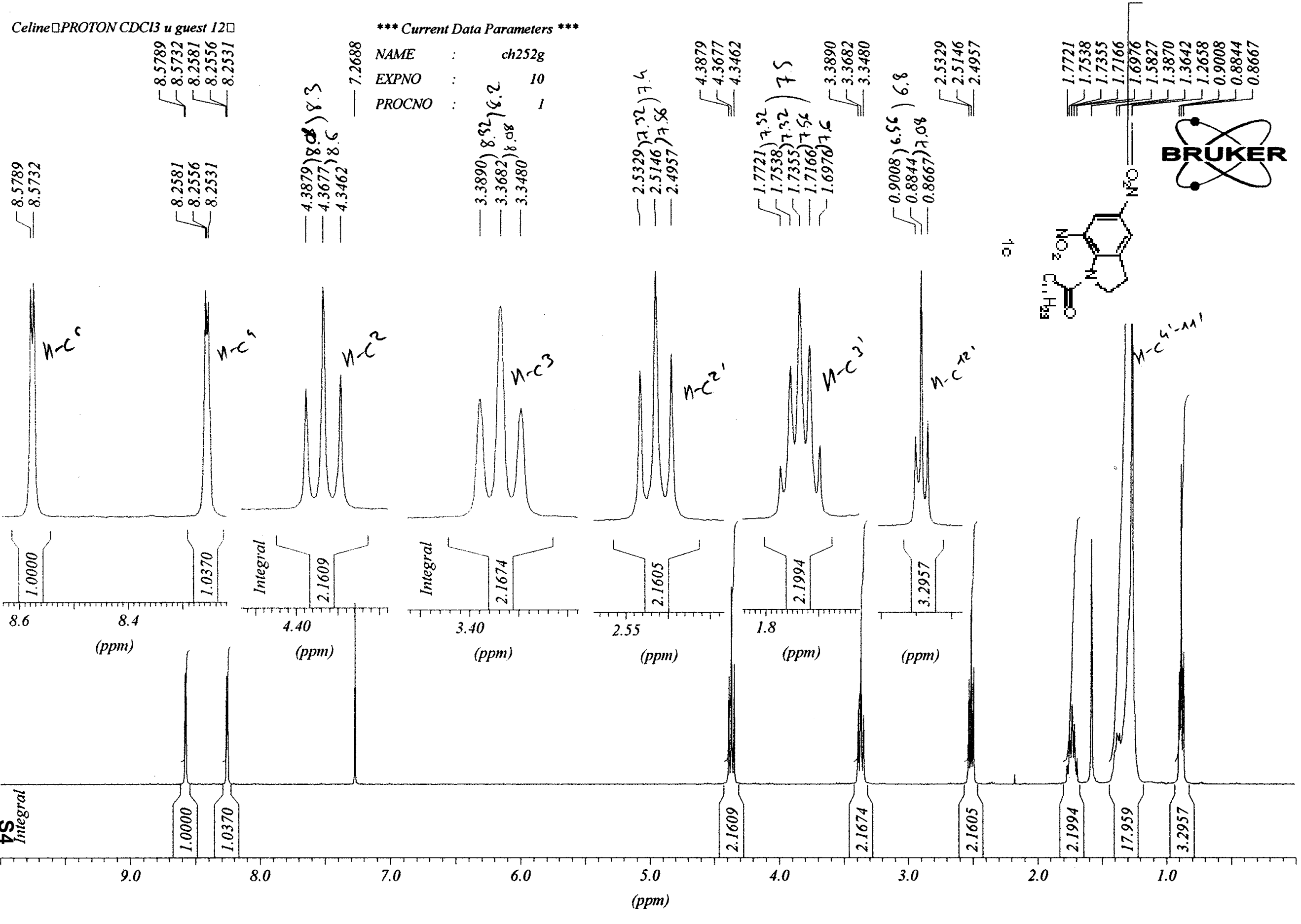


Celine

C13CPD CDC13 u guest 12
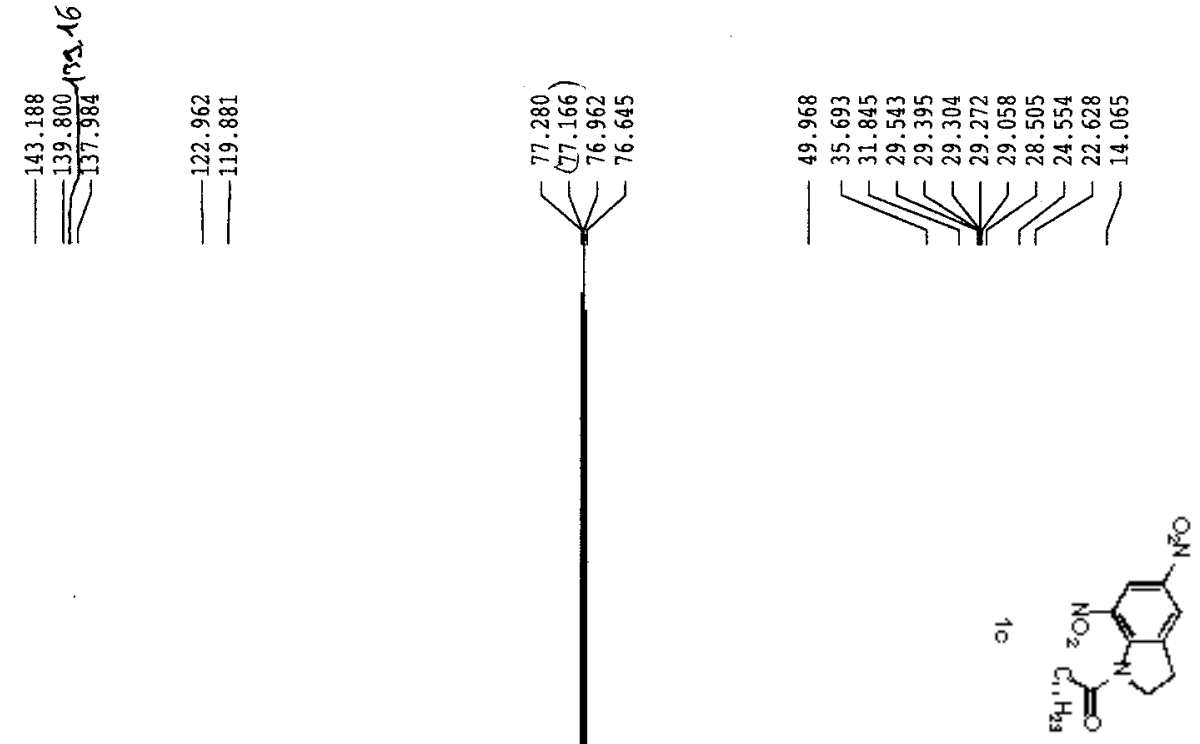

Current Data Parameters

NAME

$\mathrm{CH} 252 \mathrm{G}$

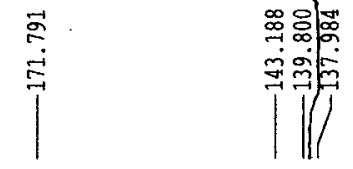

|ึ่

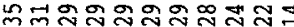

2 - Acquisition Parameters

Time

20010624

INSTRUM drx400

$5 \mathrm{~mm}$ QNP $1 \mathrm{H} /$

29pg30
65536

SOLVENT

SWH

2
SWH

$\quad 0.414641 \mathrm{~Hz}$

Al $\quad-1.2059124 \mathrm{sec}$

$\begin{array}{lr}\text { RG } & 11585.2 \\ D W & 18.400 \text { usec }\end{array}$

18.400 usec
$6.00 \mathrm{usec}$

$6.00 \mathrm{usec}$
$300.0 \mathrm{~K}$

TE

D1 $\quad 1.00000000 \mathrm{sec}$

$\mathrm{d} 12 \quad 0.00002000 \mathrm{sec}$

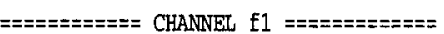

NUC1 130

$\begin{array}{ll}\text { NOC1 } & 13 \mathrm{C} \\ \text { P1 } & 7.20 \text { usec }\end{array}$

$\begin{array}{lr}\text { PL1 } & -5.00 \mathrm{~dB} \\ \text { SF01 } & 100.6237959\end{array}$

$\begin{aligned} & =====:== \pm=\text { CHANNEL } f 2 \\ & \text { waltz16 }\end{aligned}======z=====$

CPDPG

$1 \mathrm{H}$

PCPD2

PL2

PL13

80.00 usec

$13.00 \mathrm{~dB}$

$\mathrm{CM}_{2}$

$400.1316005 \mathrm{~dB}$

- Processing parameter

SF $\quad 100.6127786$

WDW

$\begin{array}{lc}\text { SSB } & 0 \\ \text { LB } & 1.00 \mathrm{~Hz}\end{array}$

GB

PC

1D NMR plot parameters

CX $\quad 30.00 \mathrm{~cm}$

F1P $\quad 240.000 \mathrm{ppm}$

$\begin{array}{lr}\text { F1 } & 24147.07 \mathrm{~Hz} \\ \text { F2P } & -10.000 \mathrm{ppm}\end{array}$

PPMCI

$\mathrm{ZZCM}$

$8.33333 \mathrm{ppm} / \mathrm{cm}$

ppm

220

200

160

140

120

100

80

60

40

20 


\section{1-Benzoyl-5,7-dinitroindoline}

\begin{tabular}{|c|c|c|c|c|c|c|c|}
\hline A cquisition $\mathrm{Tim}$ e (sec) & \multicolumn{5}{|c|}{ PROTONNR CDCl3 u jld 37} & Date & 16 Mar 2006 08:08:32 \\
\hline File $\mathrm{Name}$ & \multicolumn{5}{|c|}{ IIHOMEIDebieuxJIMy DocumentsIChimielDoctorat|NMRICH91_001001r } & Frequency $(\mathrm{MHz}$ ) & 360.13 \\
\hline Nucleus & $1 \mathrm{H}$ & Num beroftransients & 16 & origin & dpx360 & O riginalPoints Count & 16384 \\
\hline Owner & nmruser & Points Count & 16384 & Pulse Sequence & $\mathrm{zg} 30$ & ReceiverGain & 406.40 \\
\hline SW (cyclical) (Hz) & 7440.48 & Solvent & CHLOF & & & Spectrum $O$ ffset $(H z)$ & 2211.0491 \\
\hline
\end{tabular}

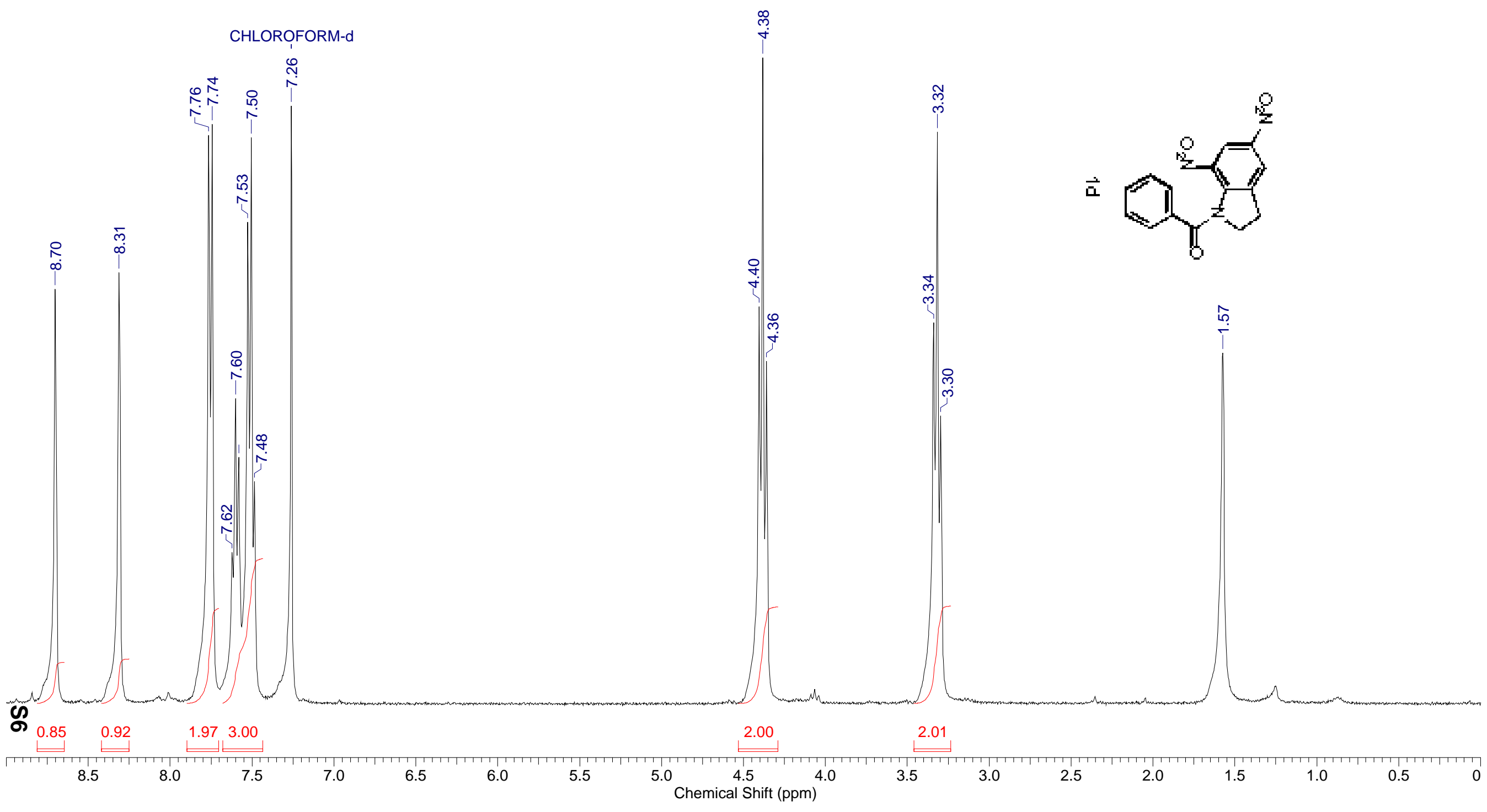




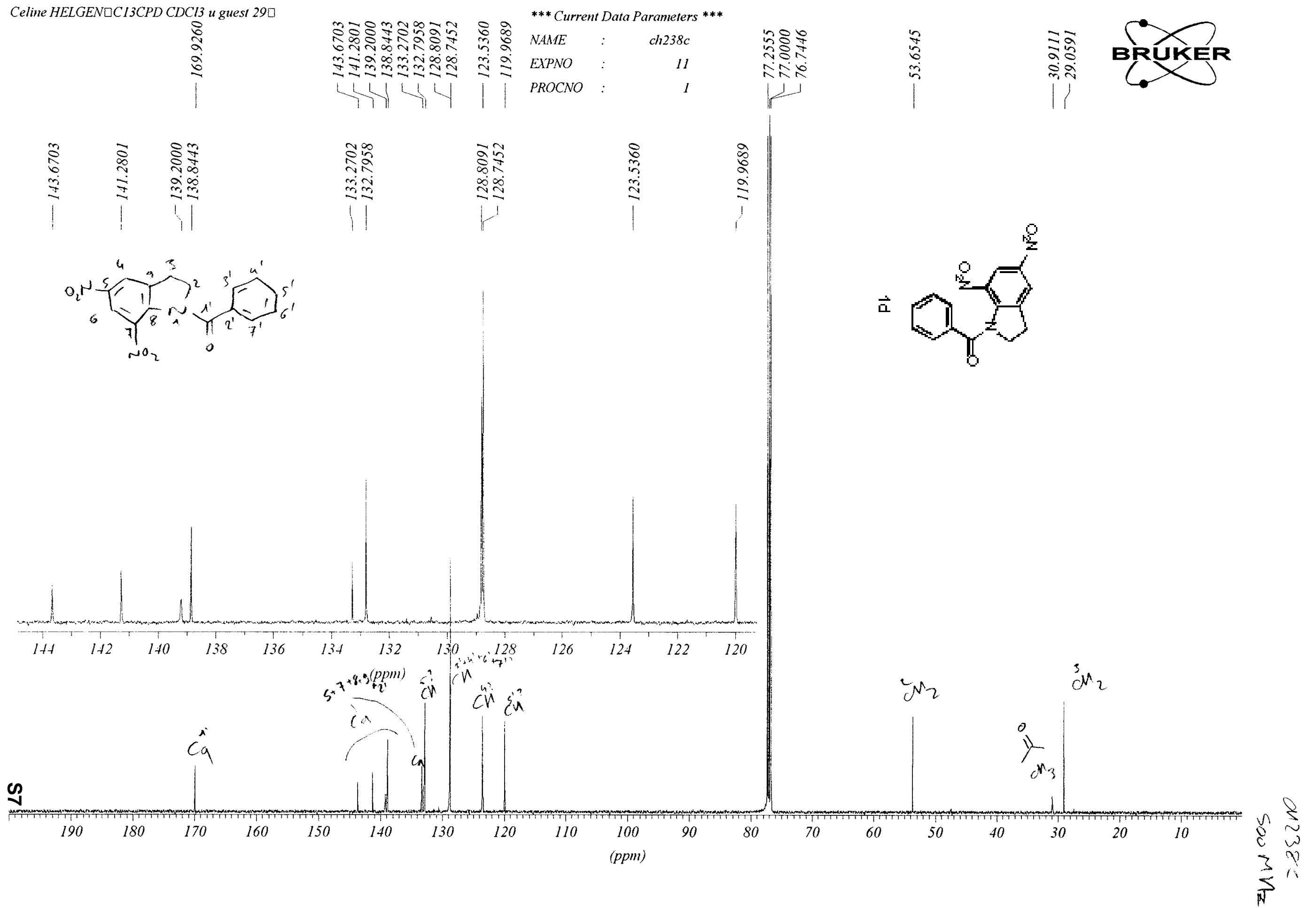


HELGEN Celine $\square$ PROTON CDC13 u guest $7 \square$

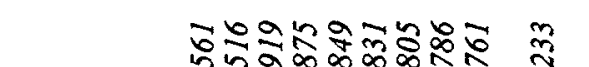

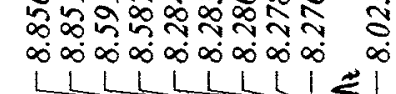
$\frac{L L L L L L L L}{N}$
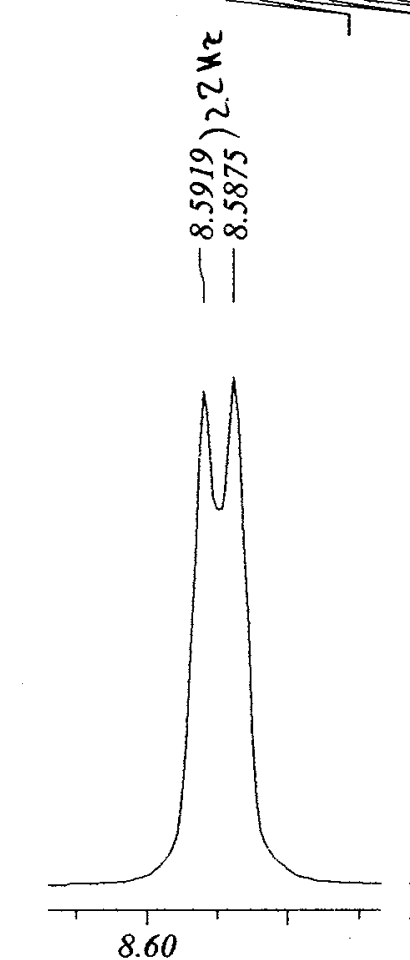

$($ ppm $)$

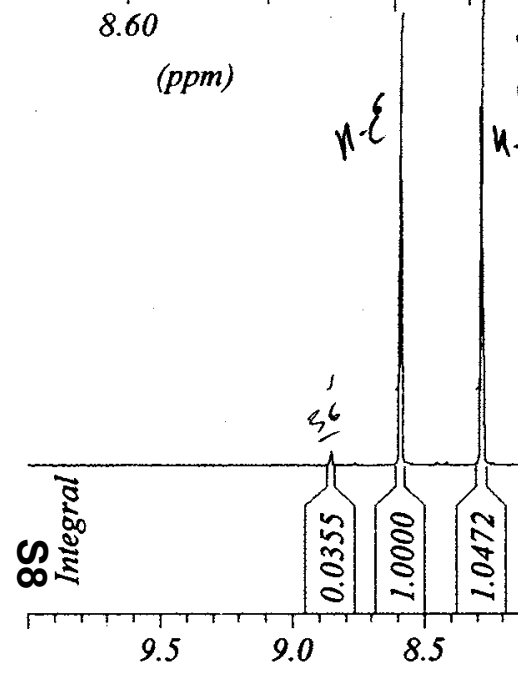

\section{*** Current Data Parameters ***}

$\pm \approx \quad$ NAME

EXPNO

PROCNO ch $253 \mathrm{~g}$

I $\xi^{\xi}$

$\infty$

Nล⿻

$\operatorname{7in} 80$

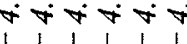

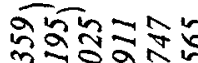

$\frac{\hbar}{5} \quad \frac{5}{5}$

害

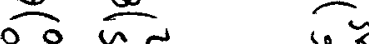
$000^{2}$

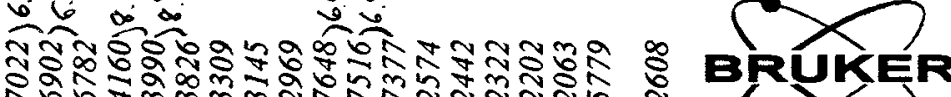
의 mmmmmmmiñर्तiñ

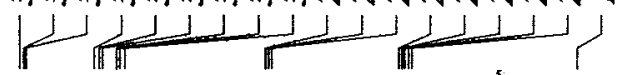

(1) $C$

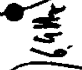

0. 0 \%

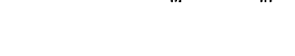<smiles>Ic1ccccc1</smiles>
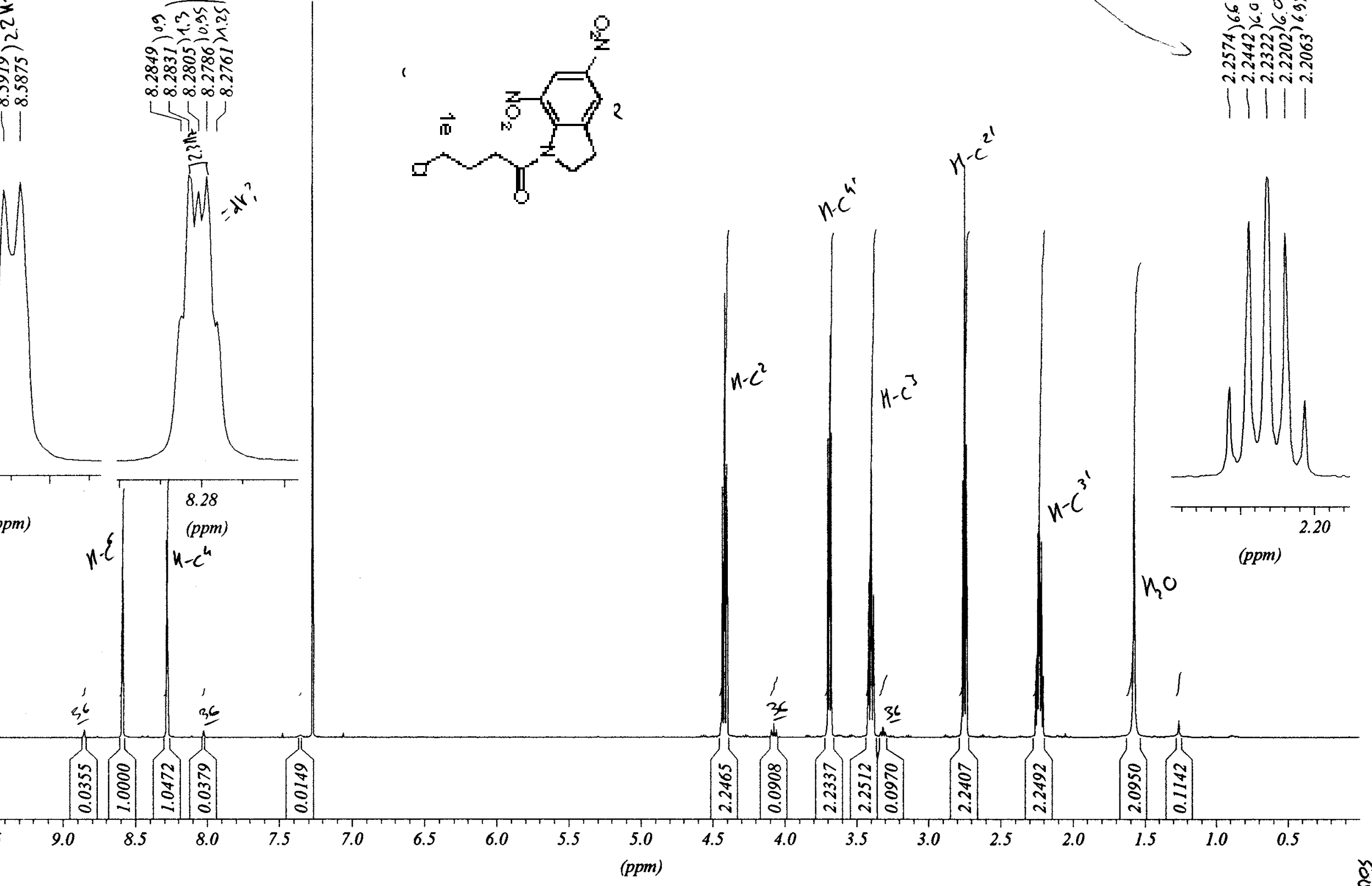

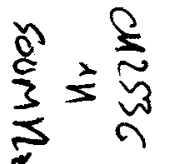


HELGEN Celine $\square C 13 C P D C D C 13$ u guest $7 \square$

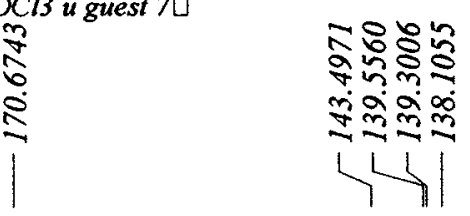

*** Current Data Parameters ***

NAME : ch253g

EXPNO

PROCNO

11

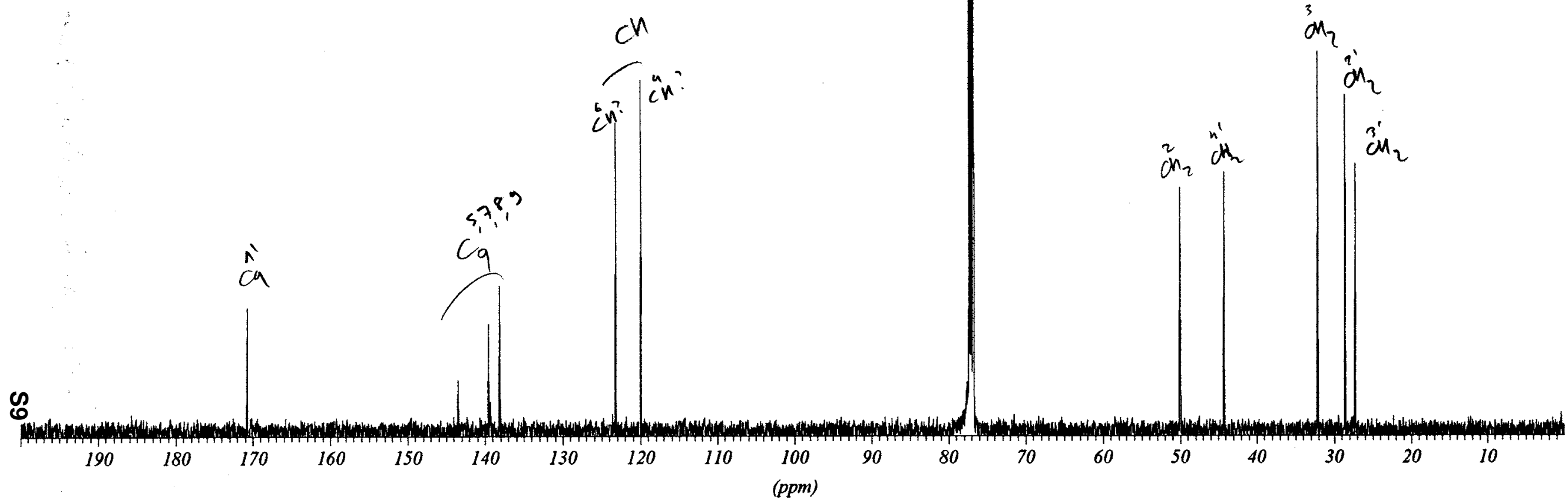

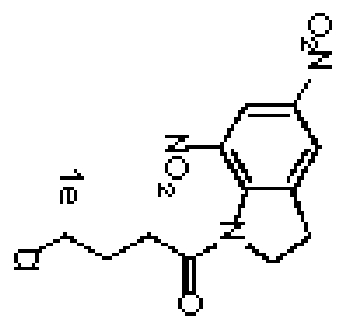

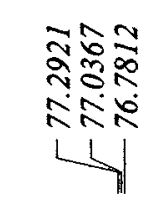

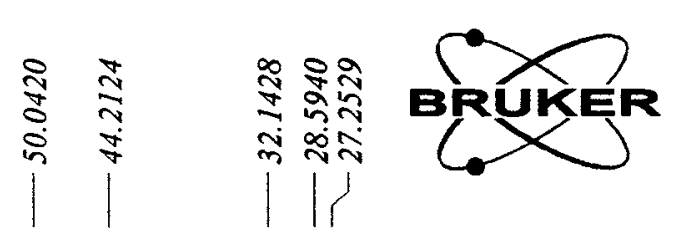

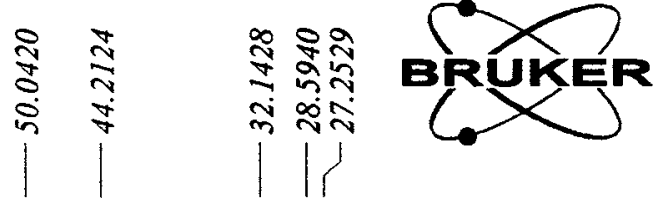

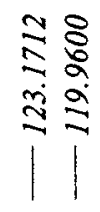

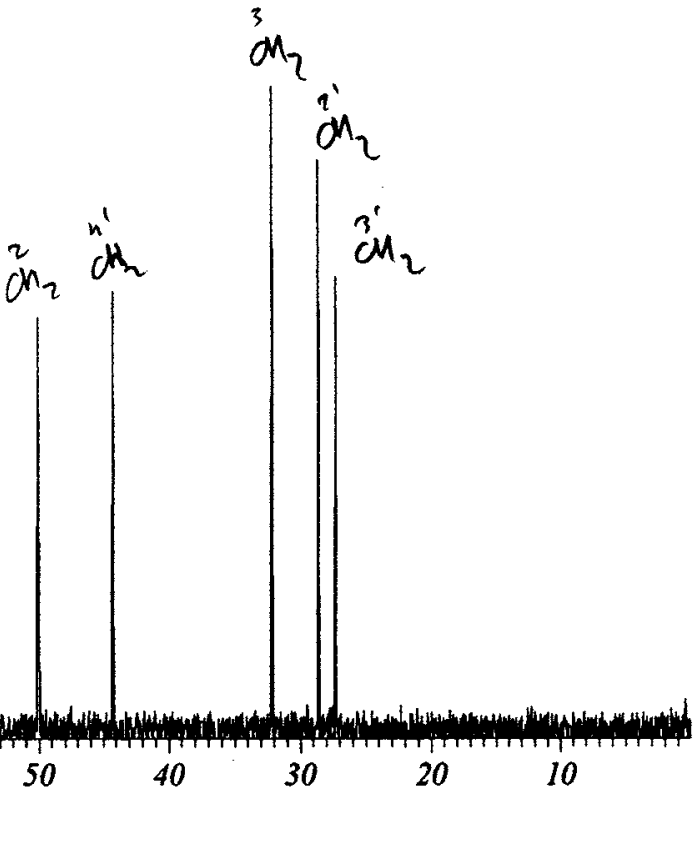




\section{1-(3-Methoxycarbonyl-propionyl)-5,7-dinitroindoline}

\begin{tabular}{|c|c|c|c|c|c|c|c|}
\hline Acquisition Tim e (sec) & 2.2021 & Com ment & \multicolumn{3}{|c|}{ PROTONNR CDCl3 u jld 6} & Date & 28 Apr 2006 13:07:12 \\
\hline File Nam e & \multicolumn{5}{|c|}{ IIHOMEIDebieuxJIMy DocumentsIChimielDoctoratINMRlind-succin_001001r } & Frequency $(\mathrm{MHz})$ & 360.13 \\
\hline Nucleus & $1 \mathrm{H}$ & Num berofTransients & 16 & O rig in & dpx360 & O riginalPoints Count & 16384 \\
\hline Owner & nmruser & Points Count & 16384 & Pulse Sequence & $\mathrm{zg} 30$ & ReceiverG ain & 456.10 \\
\hline SW (cyclical) (Hz) & 7440.48 & Solvent & \multicolumn{3}{|c|}{ CHLOROFORM-d } & Spectrum O ffset (Hz) & 2210.5947 \\
\hline
\end{tabular}

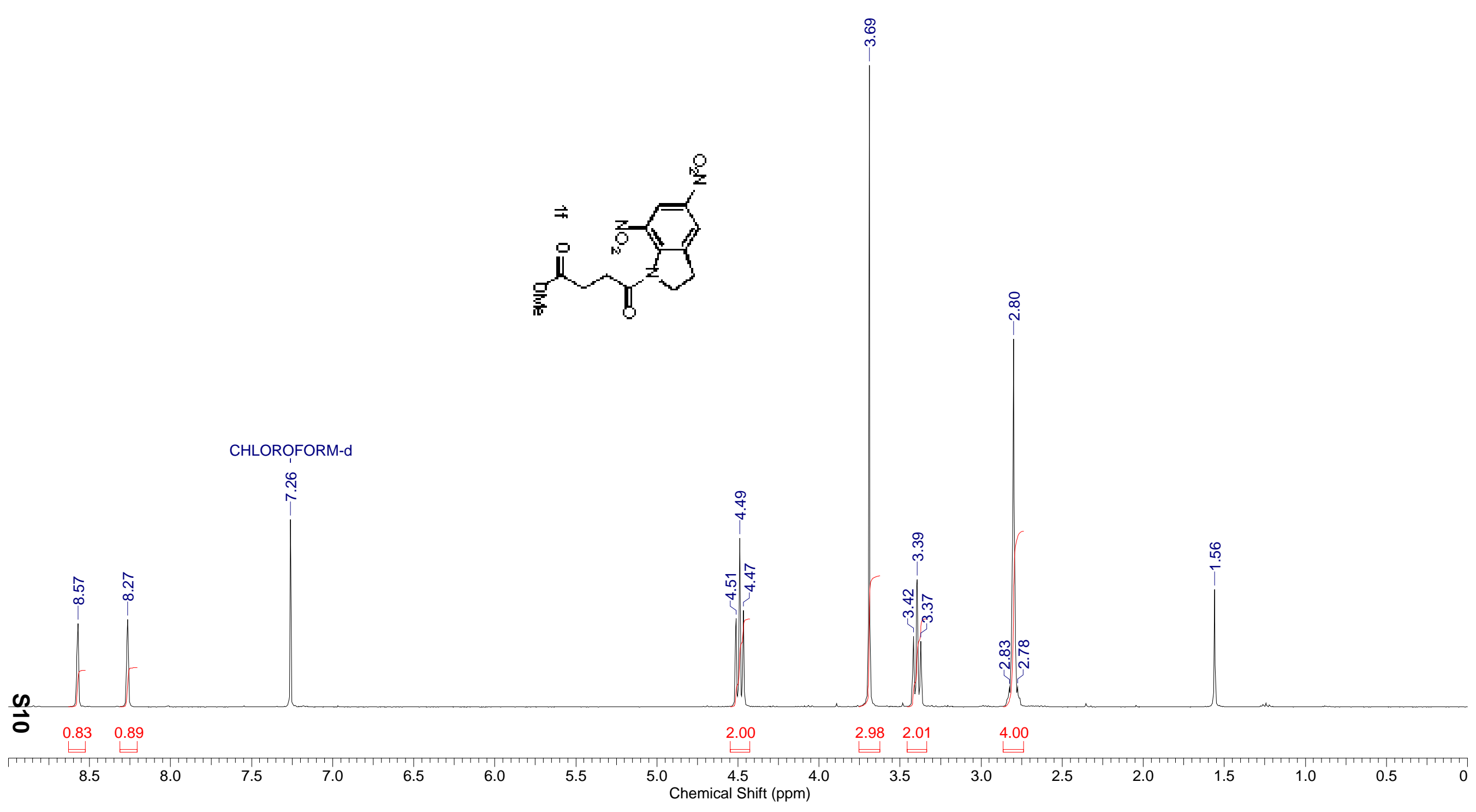




\section{jld03}

\begin{tabular}{|c|c|c|c|c|c|c|c|}
\hline Acquisition Time (sec) & 2.2021 & Comment & \multicolumn{3}{|c|}{ jld03 PROTONNR CDCl3 u jld 3} & Date & 21 Jul 2005 11:56:48 \\
\hline File Name & \multicolumn{5}{|c|}{ IIHOMEIDebieuxJIMy DocumentsIChimielDoctoratINMRVIId03 001001r } & Frequency $(\mathrm{MHz})$ & 360.13 \\
\hline Nucleus & $1 \mathrm{H}$ & Number of Transients & 32 & Origin & $\mathrm{dp} \times 360$ & Original Points Count & 16384 \\
\hline Owner & nmruser & Points Count & 16384 & Pulse Sequence & $\mathrm{zg} 30$ & Receiver Gain & 114.00 \\
\hline $\begin{array}{l}\text { SW(cyclical) }(\mathrm{Hz}) \\
\text { Sweep Width }(\mathrm{Hz})\end{array}$ & $\begin{array}{l}7440.48 \\
7440.02\end{array}$ & Solvent & \multicolumn{3}{|c|}{ CHLOROFORM-d } & Spectrum Offset $(\mathrm{Hz})$ & 2210.5950 \\
\hline
\end{tabular}

\begin{tabular}{|ll|l|}
\hline Sweep Width $(\mathrm{Hz})$ & 7440.02 & Temperature (degree C) 27.000
\end{tabular}

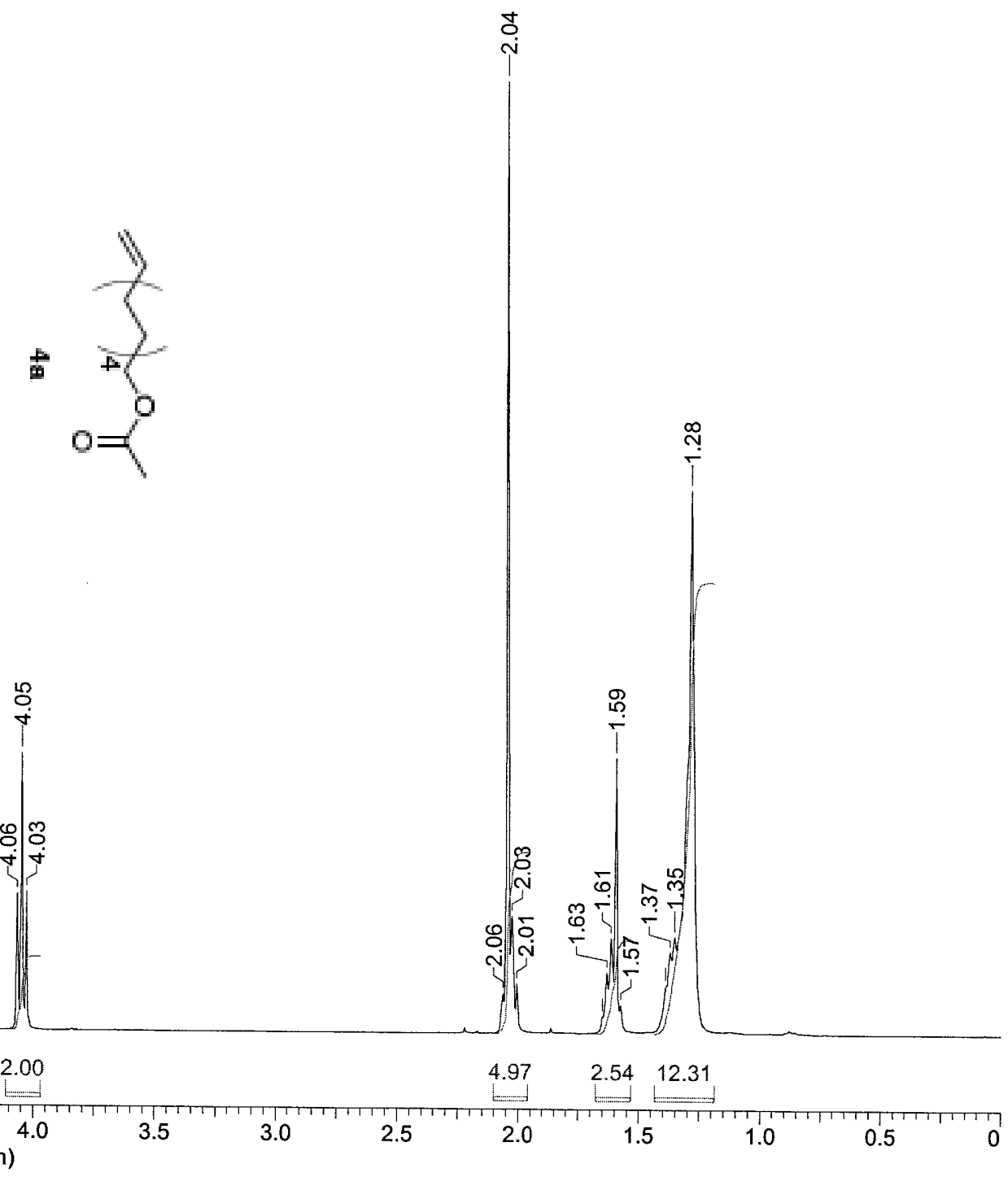




\section{jld03, C13}

\begin{tabular}{|c|c|c|c|c|c|c|c|}
\hline Acquisition Time (sec) & 1.5139 & Comment & \multirow{2}{*}{\multicolumn{3}{|c|}{$\begin{array}{l}\text { jld03, C13 C13CPD CDCl3 u jld } 3 \\
\text { ctoratiNMRlild03-C13 } 001001 \mathrm{r}\end{array}$}} & Date & 21 Jul 2005 22:04:48 \\
\hline File Name & \multicolumn{4}{|c|}{ IIHOMEIDebieuxJIMy DocumentsIChimielDoctoratINMRlild03-C13_001001r } & & Frequency $(\mathrm{MHz})$ & 90.55 \\
\hline Nucleus & $13 \mathrm{C}$ & Number of Transients & 4096 & Origin & $d p \times 360$ & Original Points Count & 32768 \\
\hline Owner & nmruser & Points Count & 32768 & Pulse Sequence & zgpg30 & Receiver Gain & 1625.50 \\
\hline SW(cyclical) $(\mathrm{Hz})$ & 21645.02 & Solvent & CHLOF & & & Spectrum Offset ( $\mathrm{Hz})$ & 9030.6260 \\
\hline
\end{tabular}

\begin{tabular}{|ll|l|l}
\hline Sweep Width $(\mathrm{Hz})$ & 21644.36 & Temperature (degree C) 27.000
\end{tabular}

CHLOROFORM-d
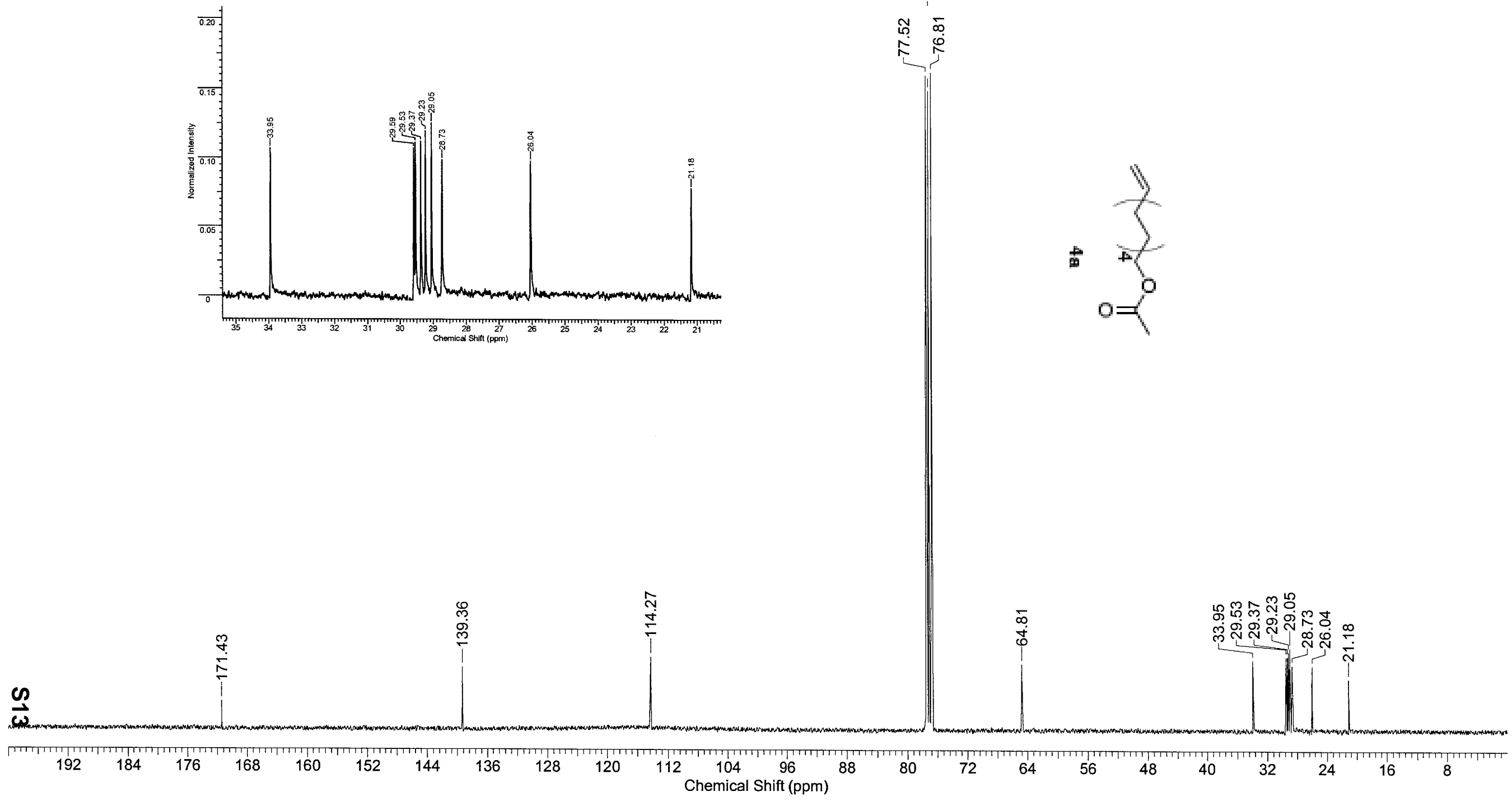


\section{jld05-1}

\begin{tabular}{|l|ll}
\hline Acquisition Time (sec) 2.2021 & Comment & jld05-1p pur PROTONNR CDCl3 u jld 9
\end{tabular} File Name $\quad$ IHOMEIDebieuxJIMy DocumentsIChimielDoctoratINMRlild05-1p 001001 \begin{tabular}{ll|l} 
Nucleus & $1 \mathrm{H}$ & Number of Transients \\
\hline
\end{tabular}

Owner Points Count 16384 Origin Solvent CHLOROFORM-d Pulse Sequence

$\mathrm{dp} \times 360$
$\mathrm{zg} 30$
Date

Frequency $\left(\mathrm{MHz}_{\mathrm{z}}\right.$

25 Jul 2005 12:07:28

$\mathrm{SW}$ (cyclical) $(\mathrm{Hz})$ 7440.48

Temperature (degree C) 27.000

Original Points Count 16384

Receiver Gain 203.20

Sweep Width $(\mathrm{Hz})$ 7440.02

I
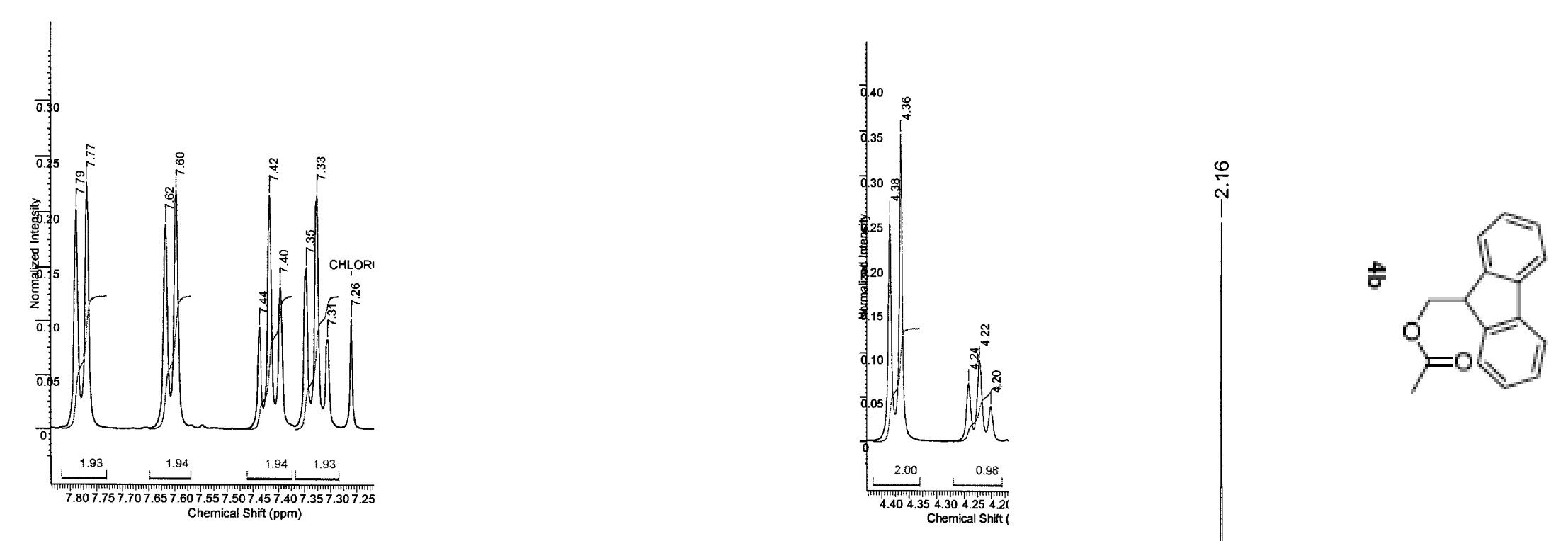

CHLOROFORM-d
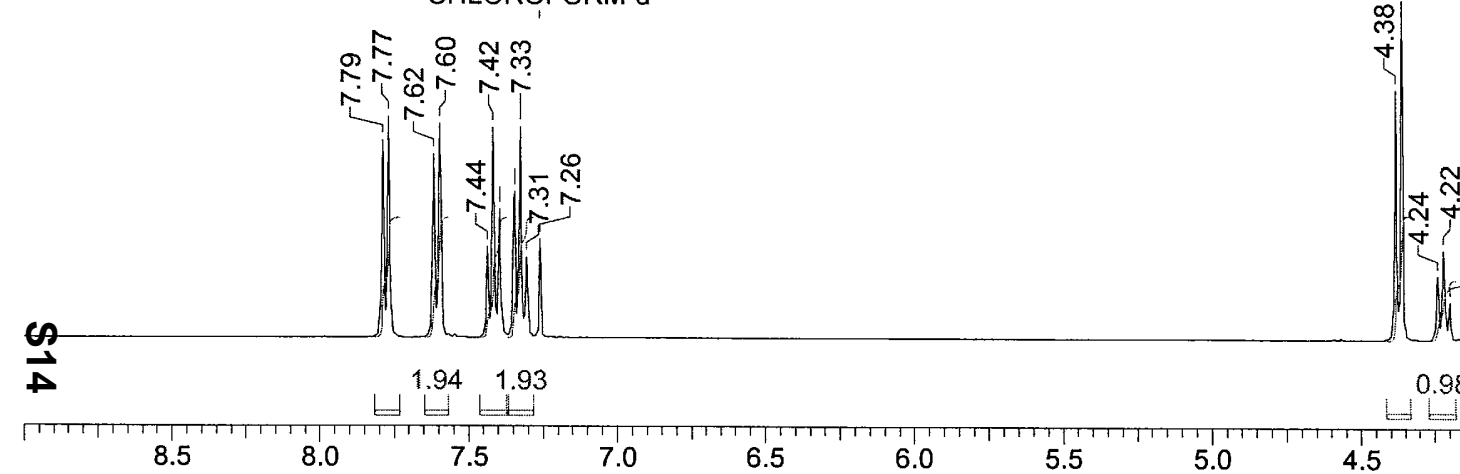

$8.5 \quad 8.0$

7.5

7.0

6.5

6.0

5.5

Chemical Shift (ppm)

4.0

3.5

2.0 3.00
$11+110$

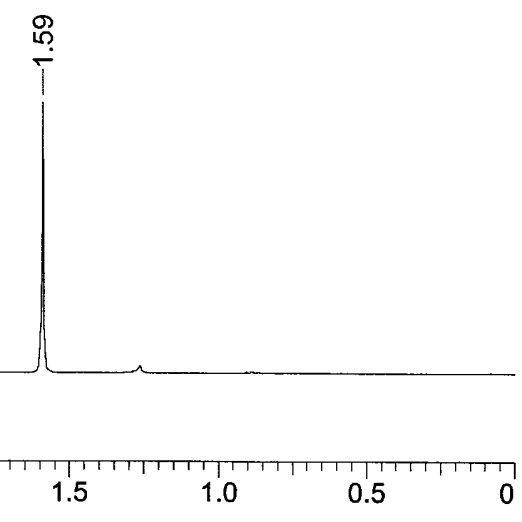




\section{jld05-2}

\begin{tabular}{|l|l|l}
\hline Acquisition Time (sec) 1.5139 & Comment & jld05-1, C13 C13CPD CDCl3 u jld 9 \\
\hline
\end{tabular}

File Name $\quad$ IIHOMEIDebieuxJIMy DocumentsIChimielDoctoratINMRlild05-1C13 001001r

\begin{tabular}{|ll|ll|ll}
\hline Nucleus & $13 \mathrm{C}$ & Number of Transients & 2048 & Origin & dpx360 \\
\hline Owner & nmruser & Points Count & 32768 & Pulse Sequence & zgpg30
\end{tabular}

Owner

Points Count

Pulse Sequence

zgpg30

Date

Frequency $(\mathrm{MHz})$

25 Jul 2005 23:19:28

SW(cyclical) $(\mathrm{Hz})$

21645.02

Solvent

CHLOROFORM-

\begin{tabular}{|lll}
\hline Sweep Width $(\mathrm{Hz})$ & 21644.36 & Temperature (degree C) 27.000 \\
\hline
\end{tabular}

Receiver Gain

32768

1625.50

CHLOROFORM-d
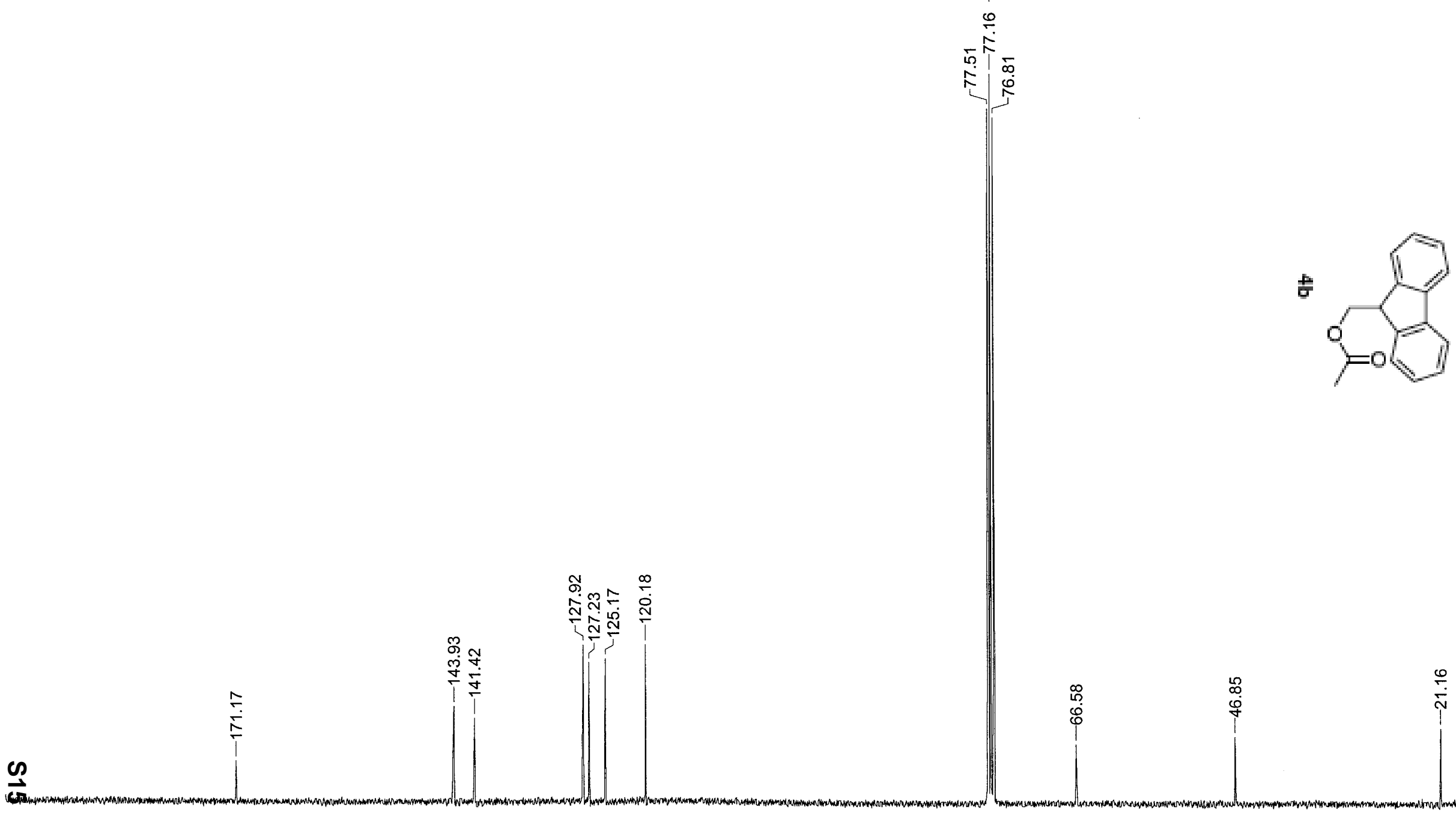

\begin{tabular}{lllllllllll|l|l|l|}
192 & 184 & 176 & 168 & 160 & 152 & 144 & 136 & 128 & 120 & 112 & 104 & 96 \\
Chemical Shift (ppm)
\end{tabular}

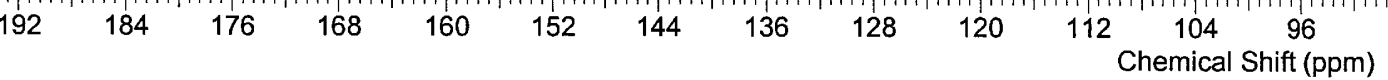

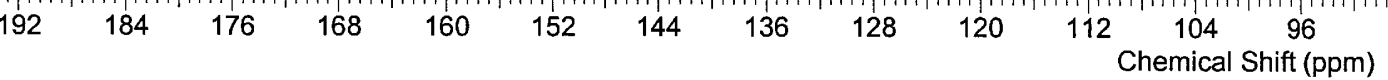

$8 \quad 80$

$00 \quad 72 \quad 64$ 


\section{jld06}

\begin{tabular}{|c|c|c|c|c|c|c|c|}
\hline Acquisition Time (sec) & 2.2021 & Comment & jld06-2 & NR CDCl3 u jld 6 & & Date & 28 Jul 2005 08:12:48 \\
\hline File Name & \multicolumn{5}{|c|}{ IIHOMEIDebieuxJIMy DocumentsIChimielDoctoratINMRIjld06-2_001001r } & Frequency $(\mathrm{MHz})$ & 360.13 \\
\hline Nucleus & $1 \mathrm{H}$ & Number of Transients & 32 & Origin & $\mathrm{dp} \times 360$ & Original Points Count & 16384 \\
\hline Owner & nmruser & Points Count & 16384 & Pulse Sequence & $\mathrm{zg} 30$ & Receiver Gain & 256.00 \\
\hline SW(cyclical) (Hz) & 7440.48 & Solvent & CHLOF & & & Spectrum Offset $(\mathrm{Hz})$ & 2210.5950 \\
\hline
\end{tabular}

Sweep Width $(\mathrm{Hz}) \quad 7440.02$ Temperature (degree C) 27.000

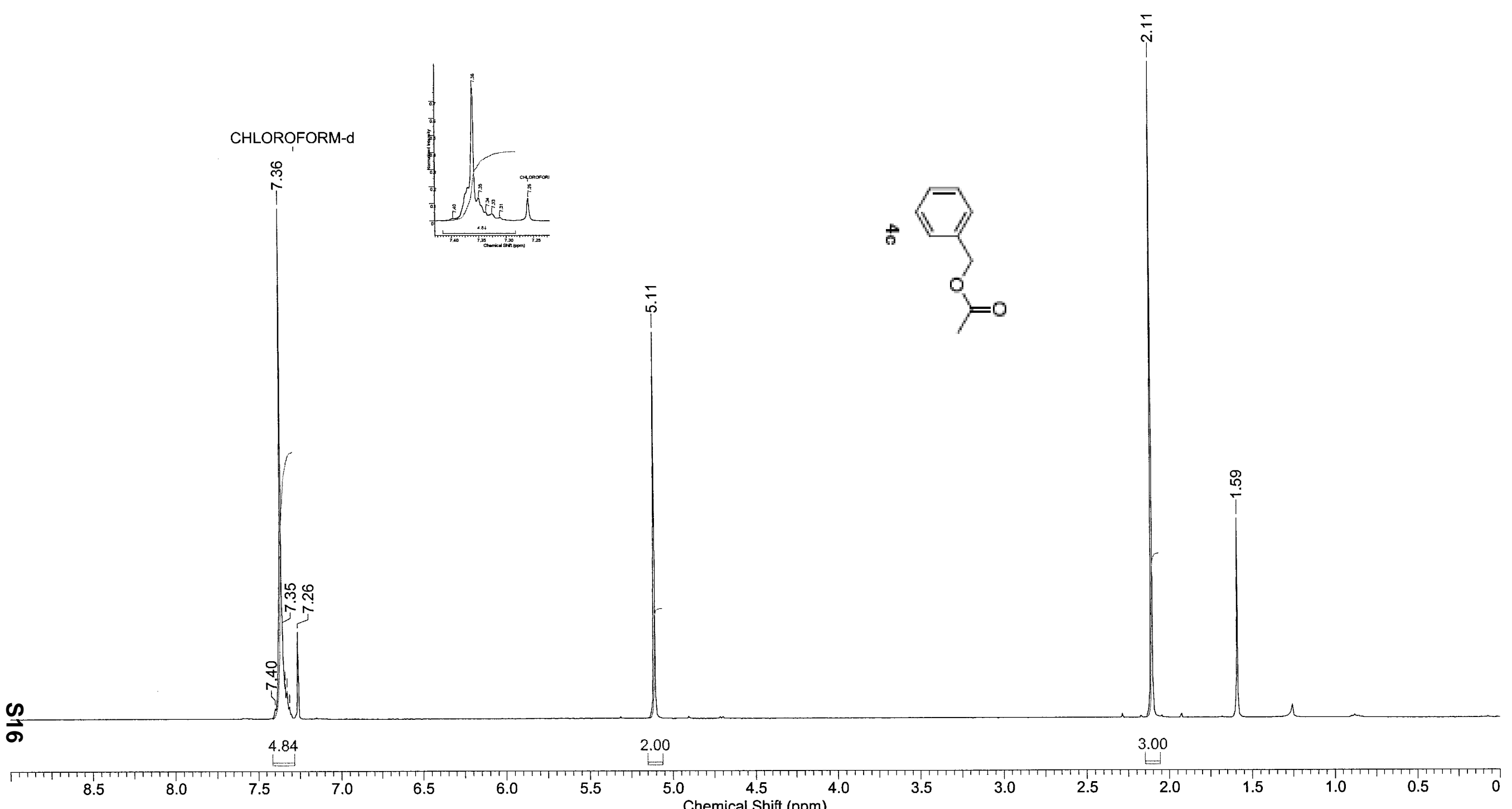




\section{jld06, C13}

\begin{tabular}{|c|c|c|c|c|c|c|c|}
\hline Acquisition Time (sec) & 1.5139 & Comment & \multicolumn{3}{|c|}{ jld06-2, C13 C13CPD CDCl3 u jld 6} & Date & 28 Jul 2005 22:04:48 \\
\hline File Name & \multicolumn{5}{|c|}{ IIHOMEIDebieuxJIMy DocumentsIChimielDoctoratINMRlild06-2_C13 001001r } & Frequency $(\mathrm{MHz})$ & 90.55 \\
\hline Nucleus & $13 \mathrm{C}$ & Number of Transients & 4096 & Origin & $\mathrm{dp} \times 360$ & Original Points Count & 32768 \\
\hline Owner & nmruser & Points Count & 32768 & Pulse Sequence & zgpg30 & Receiver Gain & 2896.30 \\
\hline SW(cyclical) $(\mathrm{Hz})$ & 21645.0 & Solvent & \multicolumn{3}{|c|}{ CHLOROFORM-d } & Spectrum Offset $(\mathrm{Hz})$ & 9030.6260 \\
\hline Sweep Width $(\mathrm{Hz})$ & 21644.3 & Temperature (degree & 27.000 & & & & \\
\hline
\end{tabular}

CHLOROFORM-d

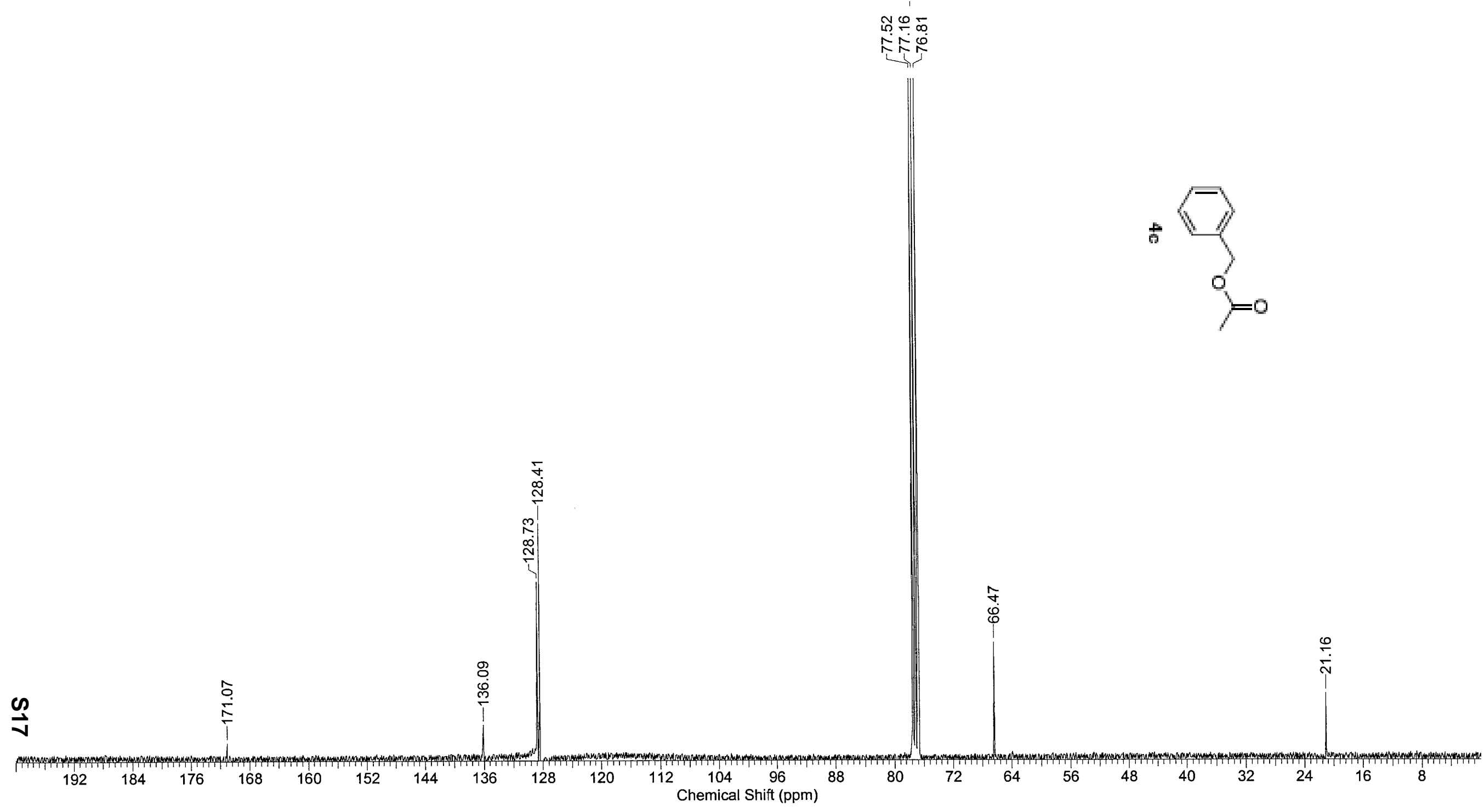




\section{jld07}

\begin{tabular}{|c|c|c|c|c|c|c|c|}
\hline Acquisition Time (sec) & 2.2021 & Comment & \multicolumn{3}{|c|}{ jld07-1 PROTONNR CDCl3 u jld 7} & Date & 29 Jul 2005 06:28:16 \\
\hline File Name & \multicolumn{5}{|c|}{ IIHOMEIDebieuxJIMy DocumentsIChimielDoctoratINMRljld07-1_001001r } & Frequency $(\mathrm{MHz})$ & 360.13 \\
\hline Nucleus & $1 \mathrm{H}$ & Number of Transients & 32 & Origin & $\mathrm{dp} \times 360$ & Original Points Count & 16384 \\
\hline Owner & nmruser & Points Count & \multirow{2}{*}{\multicolumn{3}{|c|}{ CHLOROFORM-d }} & Receiver Gain & 228.10 \\
\hline SW(cyclical) $(\mathrm{Hz})$ & 7440.48 & Solvent & & & & Spectrum Offset $(\mathrm{Hz})$ & 2210.5950 \\
\hline
\end{tabular}

Temperature (degree C) 27.000

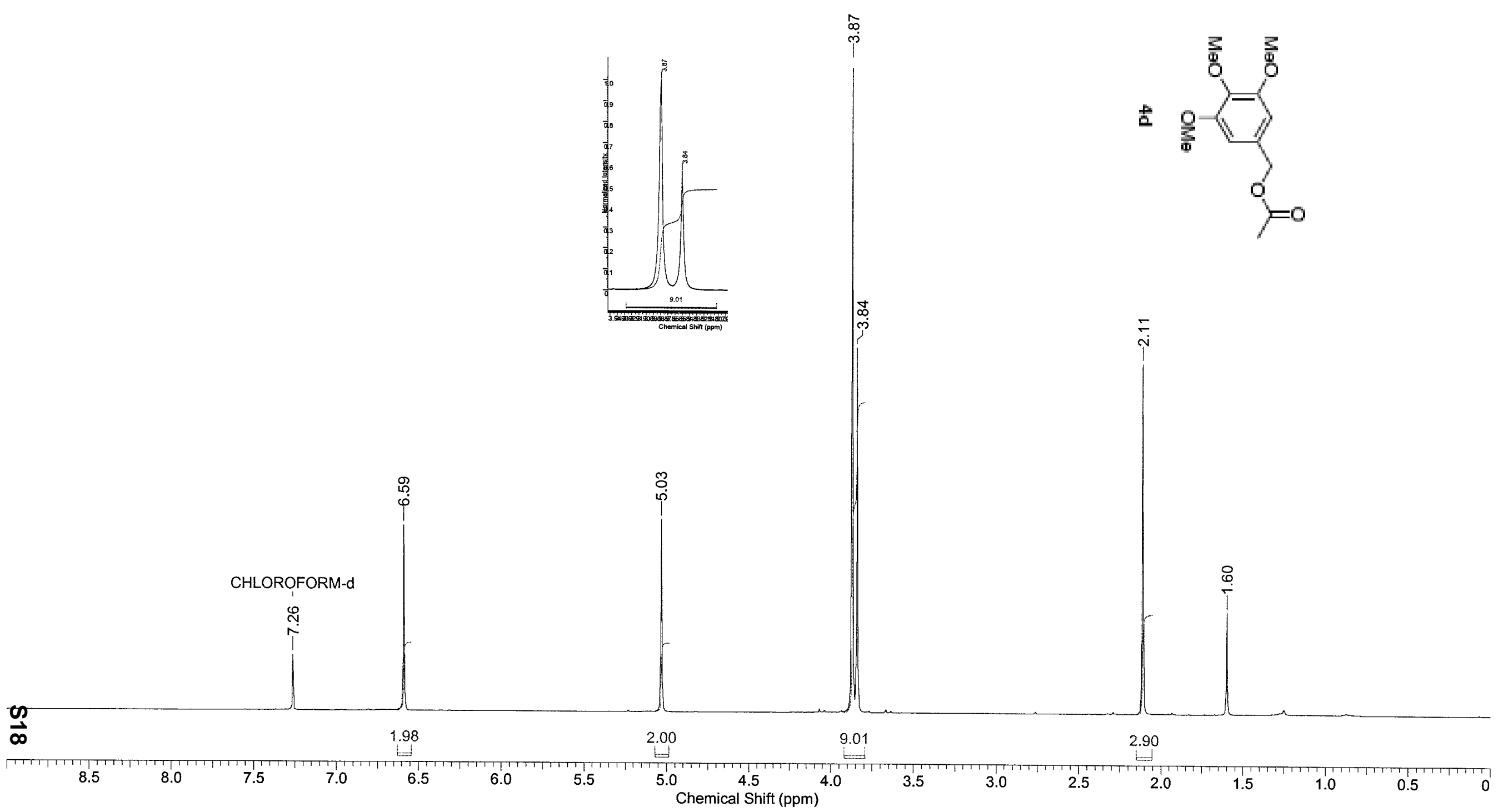




\section{jld07, C13}

\begin{tabular}{|c|c|c|c|c|c|c|c|}
\hline Acquisition Time (sec) & 1.5139 & Comment & \multicolumn{3}{|c|}{ jld07-1, C13 C13CPD CDCl3 u jld 7} & Date & 30 Jul 2005 01:12:32 \\
\hline File Name & \multicolumn{5}{|c|}{ IIHOMEIDebieuxJIMy DocumentsIChimielDoctoratINMRIjld07-1_C13_001001r } & Frequency $(\mathrm{MHz})$ & 90.55 \\
\hline Nucleus & $13 \mathrm{C}$ & Number of Transients & 4096 & Origin & $\mathrm{dp} \times 360$ & \multicolumn{2}{|c|}{ Original Points Count 32768} \\
\hline Owner & nmruser & Points Count & 32768 & Pulse Sequence & zgpg30 & Receiver Gain & 1448.20 \\
\hline SW(cyclical) $(\mathrm{Hz})$ & 21645.02 & Solvent & \multicolumn{3}{|c|}{ CHLOROFORM-d } & Spectrum Offset $(\mathrm{Hz})$ & 9029.9648 \\
\hline
\end{tabular}

\begin{tabular}{ll|l} 
Sweep Width $(\mathrm{Hz})$ & 21644.36 & Temperature (degree C) 27.000
\end{tabular}

CHLOROFORM-d
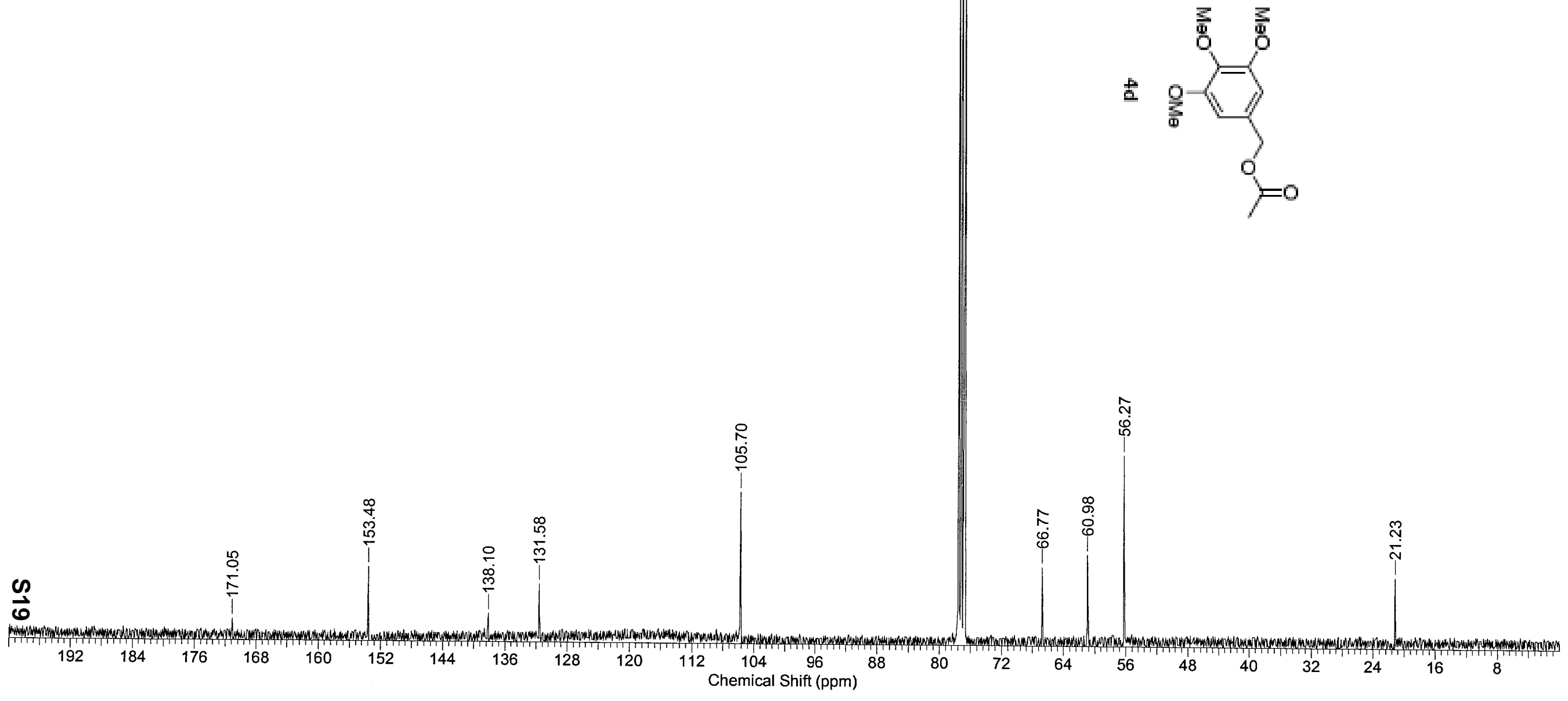


\section{jld10}

\begin{tabular}{|c|c|c|c|c|c|c|c|}
\hline Acquisition Time (sec) & 2.2021 & Comment & \multicolumn{3}{|c|}{ jld10 PROTONNR CDCl3 u jld 8} & Date & 06 Aug 2005 08:12:48 \\
\hline File Name & \multicolumn{5}{|c|}{ IIHOMEIDebieuxJIMy Documents IChimielDoctoratINMRIjld10 $00100 \mathrm{ir}$} & Frequency $(\mathrm{MHz})$ & 360.13 \\
\hline Nucleus & $1 \mathrm{H}$ & Number of Transients & 32 & Origin & $\mathrm{dp} \times 360$ & Original Points Count & 16384 \\
\hline Owner & nmruser & Points Count & 16384 & Pulse Sequence & $\mathrm{zg} 30$ & Receiver Gain & 143.70 \\
\hline SW(cyclical) $(\mathrm{Hz})$ & 7440.48 & Solvent & \multicolumn{3}{|c|}{ CHLOROFORM-d } & Spectrum Offset $(\mathrm{Hz})$ & 2210.5950 \\
\hline
\end{tabular}

(Hz) $\quad 7440.02$

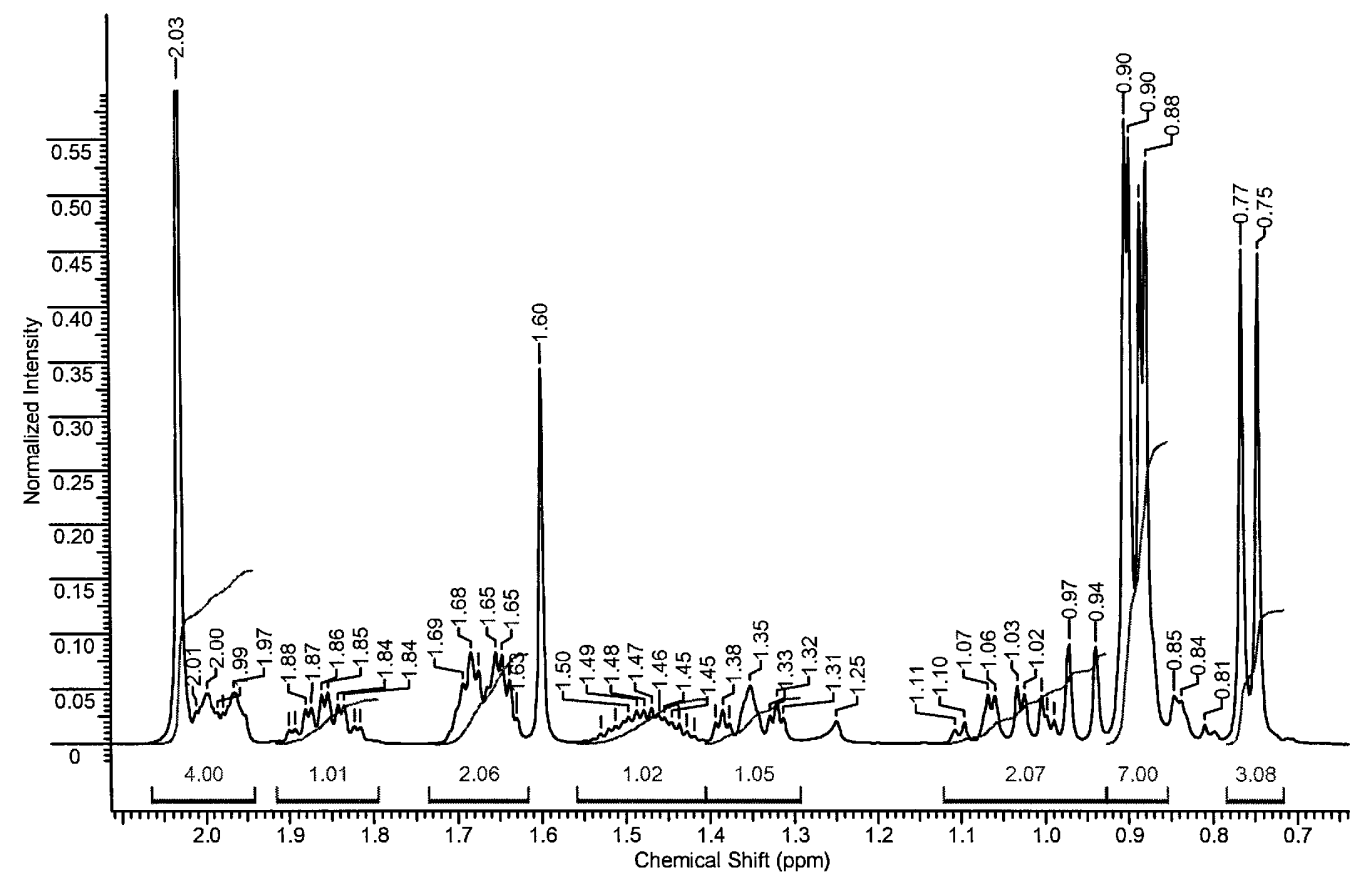

CHLOROFORM-d

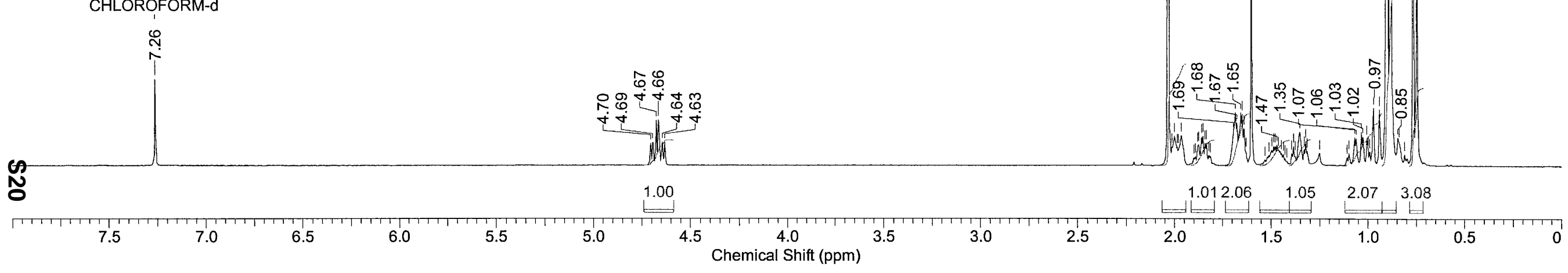




\section{jld10, C13}

\begin{tabular}{|c|c|c|c|c|c|c|c|}
\hline Acquisition Time (sec) & 1.5139 & Comment & \multicolumn{3}{|c|}{ jld10, C13 C13CPD CDCl3 u jld 8} & Date & 06 Aug 2005 11:18:24 \\
\hline File Name & \multicolumn{5}{|c|}{ IIHOMEIDebieuxJIMy DocumentsIChimielDoctoratINMRIjId10_C13_001001r } & Frequency $(\mathrm{MHz})$ & 90.55 \\
\hline Nucleus & $13 \mathrm{C}$ & Number of Transients & 3072 & Origin & $d p \times 360$ & Original Points Count & 32768 \\
\hline Owner & nmruser & Points Count & 32768 & Pulse Sequence & zgpg30 & Receiver Gain & 1824.60 \\
\hline$S W$ (cyclical) $(H z)$ & 21645.02 & Solvent & CHLOF & & & Spectrum Offset ( $\mathrm{Hz})$ & 9016.1367 \\
\hline
\end{tabular}

CHLOROFORM-d

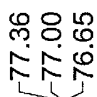
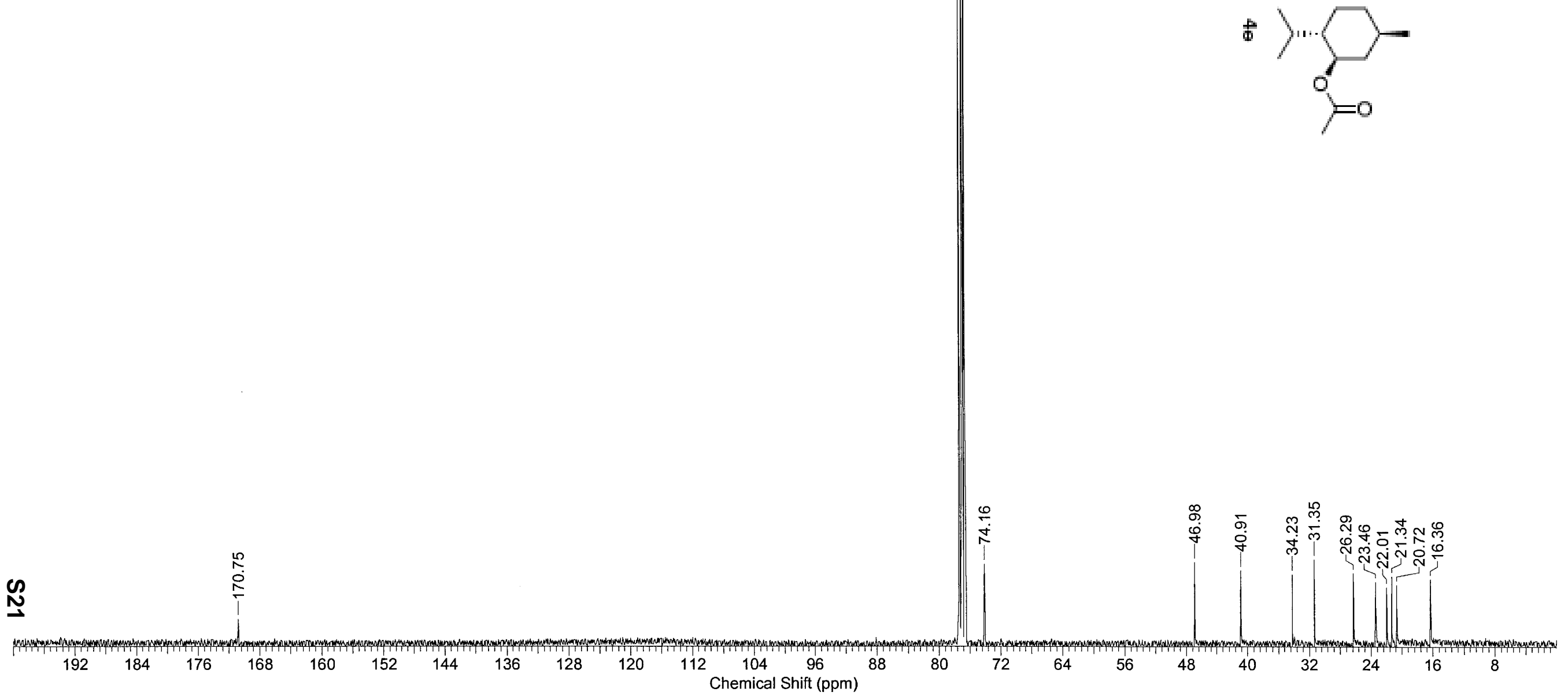


\section{jld11-1}

\begin{tabular}{|c|c|c|c|c|c|c|c|}
\hline Acquisition Time (sec) & \multirow{2}{*}{\multicolumn{5}{|c|}{\begin{tabular}{l|ll}
2.2021 & Comment & jld11-1 PROTONNR CDCl3 u jld 11 \\
IIHOMEIDebieuxJIMy DocumentsiChimielDoctoratWMRlild11-1_001001r
\end{tabular}}} & Date & 09 Aug 2005 11:50:24 \\
\hline File Name & & & & & & Frequency $(\mathrm{MHz})$ & 360.13 \\
\hline Nucleus & $1 \mathrm{H}$ & Number of Transients & 32 & Origin & $\mathrm{dp} \times 360$ & \multicolumn{2}{|c|}{ Original Points Count 16384} \\
\hline Owner & nmruser & Points Count & 16384 & Pulse Sequence & $\mathrm{zg} 30$ & Receiver Gain & 181.00 \\
\hline $\begin{array}{l}\text { SW(cyclical) }(\mathrm{Hz}) \\
\text { Sweep Width }(\mathrm{Hz})\end{array}$ & $\begin{array}{l}7440.48 \\
7440.02\end{array}$ & $\begin{array}{l}\text { Solvent } \\
\text { Temperature (degree C }\end{array}$ & \multicolumn{3}{|c|}{ CHLOROFORM-d } & Spectrum Offset $(\mathrm{Hz})$ & 2210.5950 \\
\hline
\end{tabular}

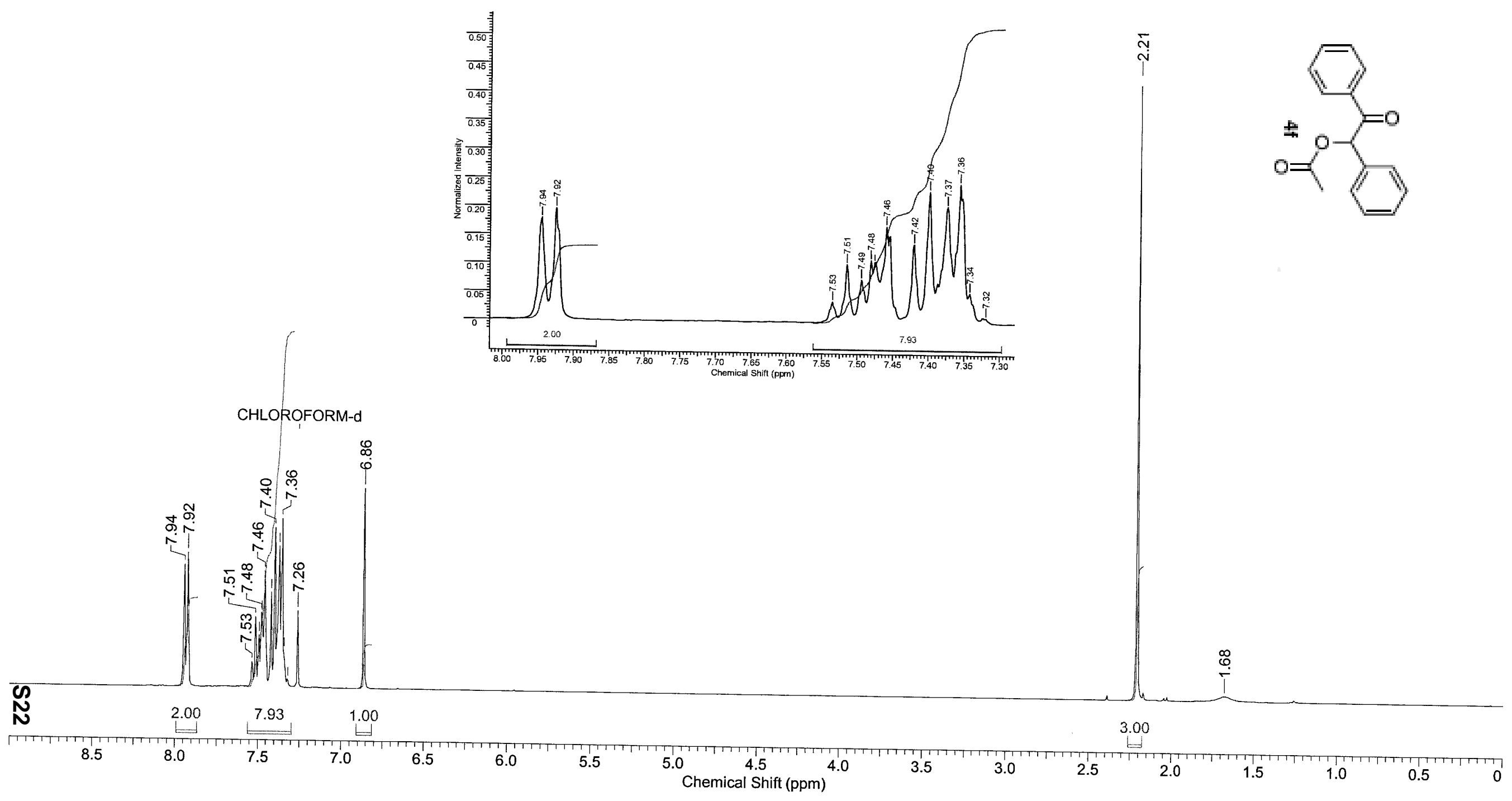




\section{jld11, C13}

\begin{tabular}{|c|c|c|c|c|c|c|c|}
\hline Acquisition Time (sec) & 1.5139 & Comment & \multirow{2}{*}{\multicolumn{3}{|c|}{$\begin{array}{l}\text { jld11, C13 C13CPD CDCl3 u jld } 11 \\
\text { toratWMRlild11-1 C13 001001r }\end{array}$}} & Date & 10 Aug 2005 21:05:04 \\
\hline File Name & \multicolumn{3}{|c|}{$\begin{array}{l}\text { IIHOMEIDebieuxJIMy Documents IChimielDoctoratINMRljld11-1_C13_001001r } \\
13 \mathrm{C}\end{array}$} & & & Frequency $(\mathrm{MHz})$ & 90.55 \\
\hline Nucleus & $13 C$ & Number of Transients & 3072 & Origin & $d p \times 360$ & Original Points Count & 32768 \\
\hline Owner & nmruser & Points Count & 32768 & Pulse Sequence & zgpg30 & Receiver Gain & 4096.00 \\
\hline $\begin{array}{l}\text { SW(cyclical) }(\mathrm{Hz}) \\
\text { Sweep Width }(\mathrm{Hz})\end{array}$ & $\frac{21645.02}{21644.36}$ & Solvent & \multicolumn{3}{|c|}{ CHLOROFORM-d } & Spectrum Offset $(\mathrm{Hz})$ & 9014.8154 \\
\hline
\end{tabular}

$\begin{array}{lll}\text { Sweep Width }(\mathrm{Hz}) & 21644.36 & \text { Temperature (degree C) } 27.000\end{array}$

CHLOROFORM-d
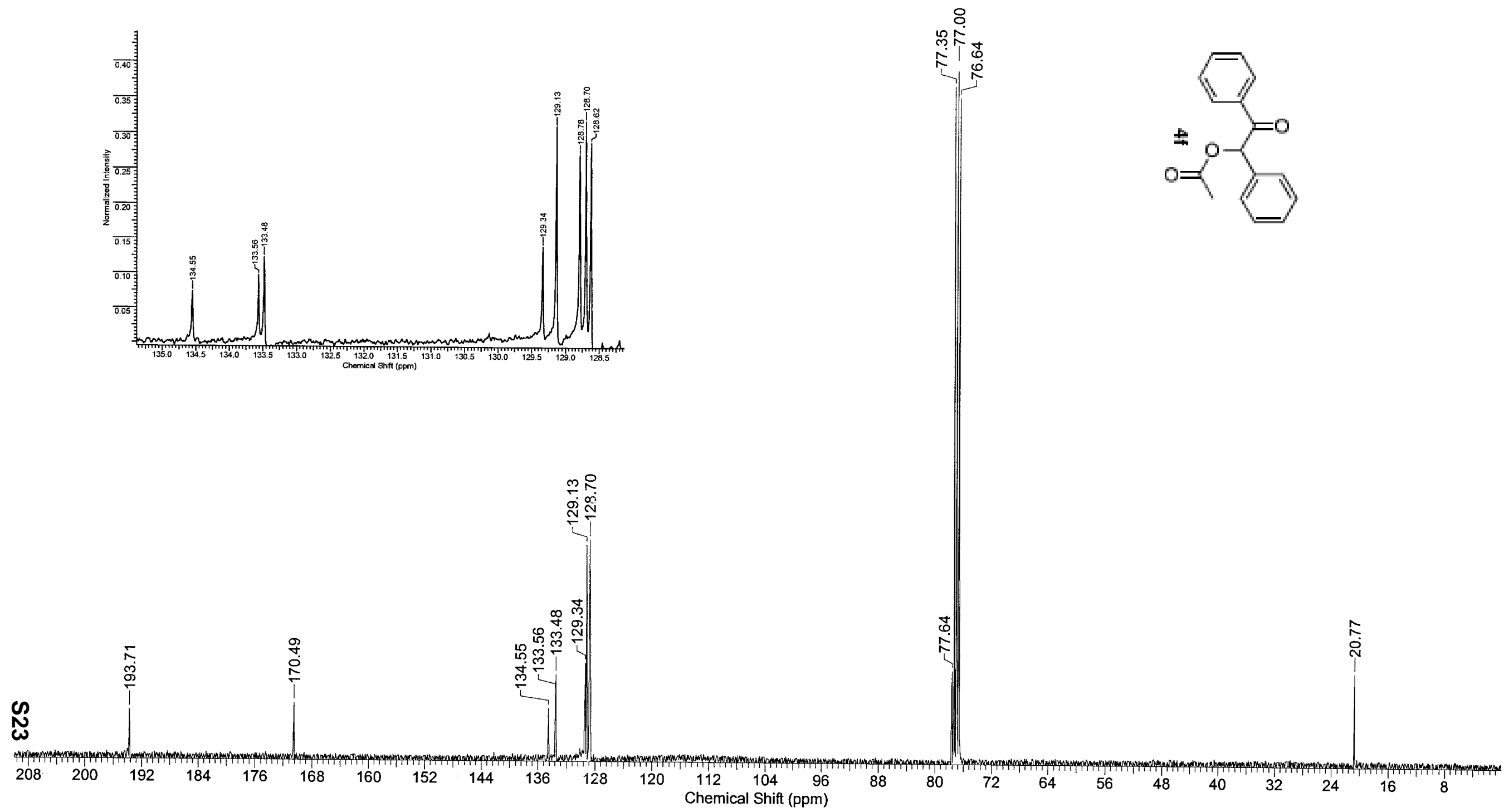


\section{jld12}

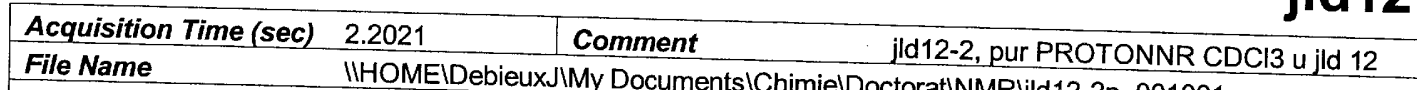

Nucleus $\quad$ IIHOMEIDebieuxJIMy Documents IChimielDoctoratiNMRljld12-2p_001001r $1 \mathrm{H}$

Number of Transients 32

$32 \quad$ Origin

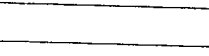

Owner

nmruser

Points Count

\begin{tabular}{l|l}
16384 & Pulse Sequence
\end{tabular}

dpx360

Date

Frequency $(\mathrm{MHz})$

13 Aug 2005 09:36:00

\begin{tabular}{l} 
Owner \\
\hline SW(cyclical) $(\mathrm{Hz})$
\end{tabular}

Solvent CHLOROFORM-d

Original Points Count 16384

\begin{tabular}{ll}
\hline Sweep Width $(\mathrm{Hz})$ & 7440.48 \\
\hline
\end{tabular} Temperature (degree C) 27.000

Receiver Gain $\quad 362.00$
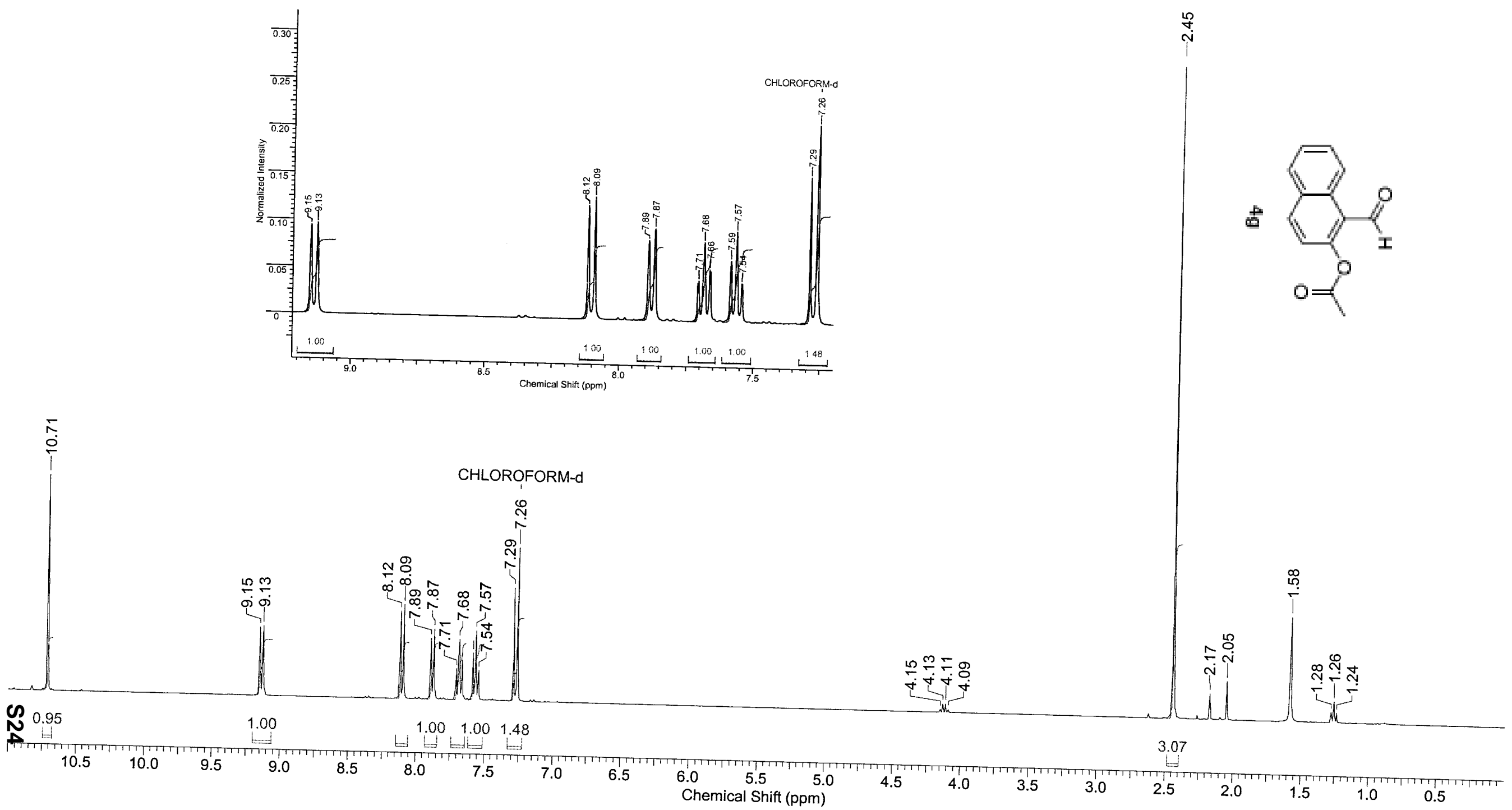


\section{jld12, C13}

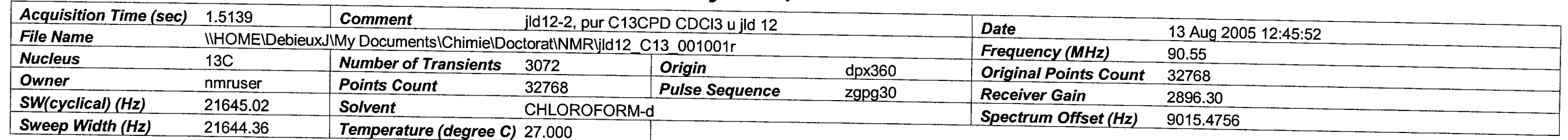

\section{CHLOROFORM-d}

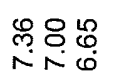

余余

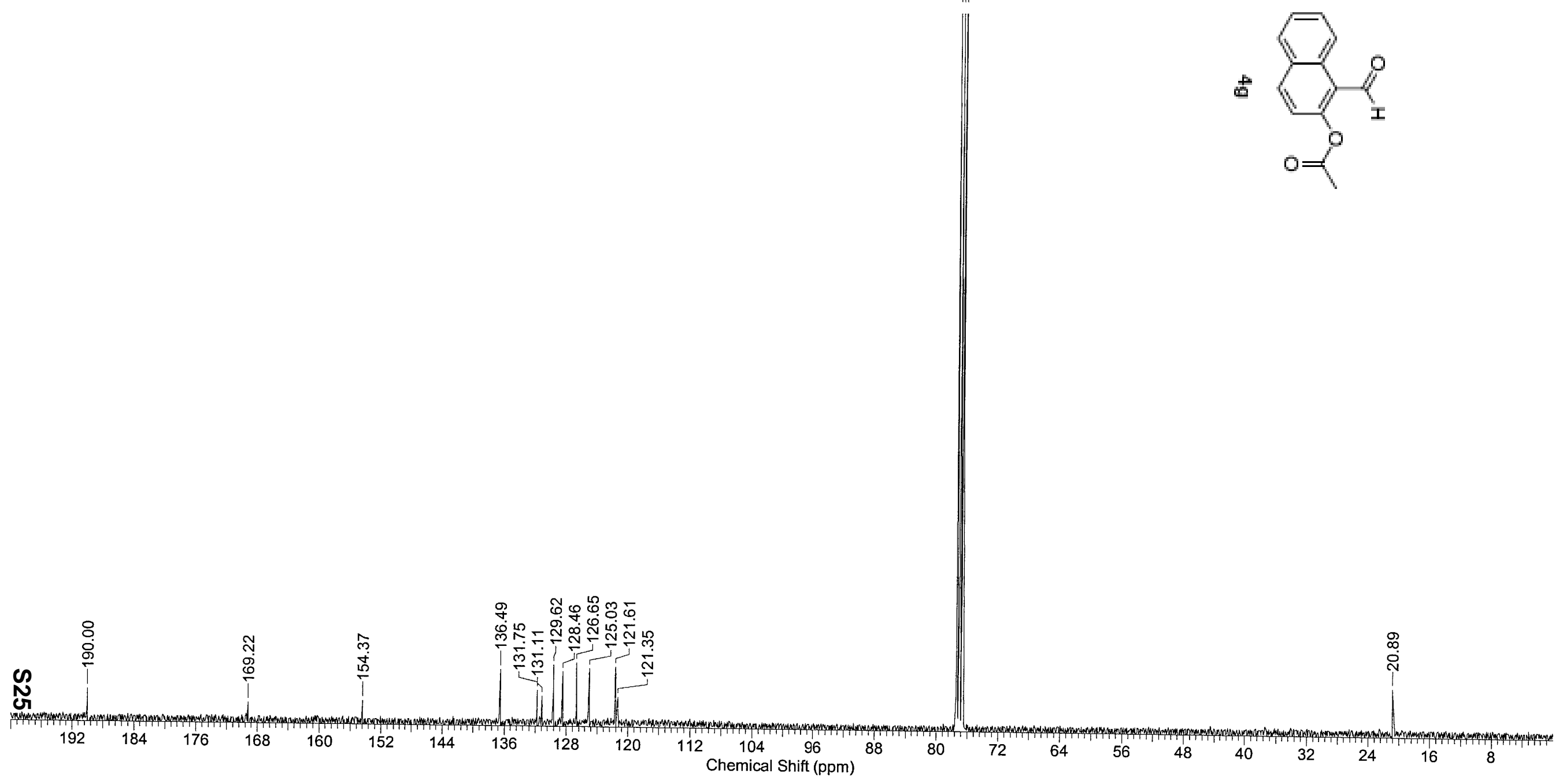




\section{jld13}

\begin{tabular}{|c|c|c|c|c|c|c|c|}
\hline Acquisition Time (sec) & 2.2021 & Comment & jld13-2, & CONNR CDCl3 u jld & & Date & 18 Aug 2005 09:57:20 \\
\hline File Name & IIHOMEID & My Documents IChimielD & ctoratiNi & p_001001r & & Frequency $(\mathrm{MHz})$ & 360.13 \\
\hline Nucleus & $1 \mathrm{H}$ & Number of Transients & 32 & Origin & $\mathrm{dp} \times 360$ & Original Points Count & 16384 \\
\hline Owner & nmruser & Points Count & 16384 & Pulse Sequence & $\mathrm{zg} 30$ & Receiver Gain & 228.10 \\
\hline$\frac{\text { SW(cyclical) }(\mathrm{Hz})}{\text { Sweep Width }(\mathrm{Hz})}$ & $\begin{array}{l}7440.48 \\
7440.02\end{array}$ & Solvent & CHLOF & & & Spectrum Offset $(\mathrm{Hz})$ & 2210.5950 \\
\hline
\end{tabular}

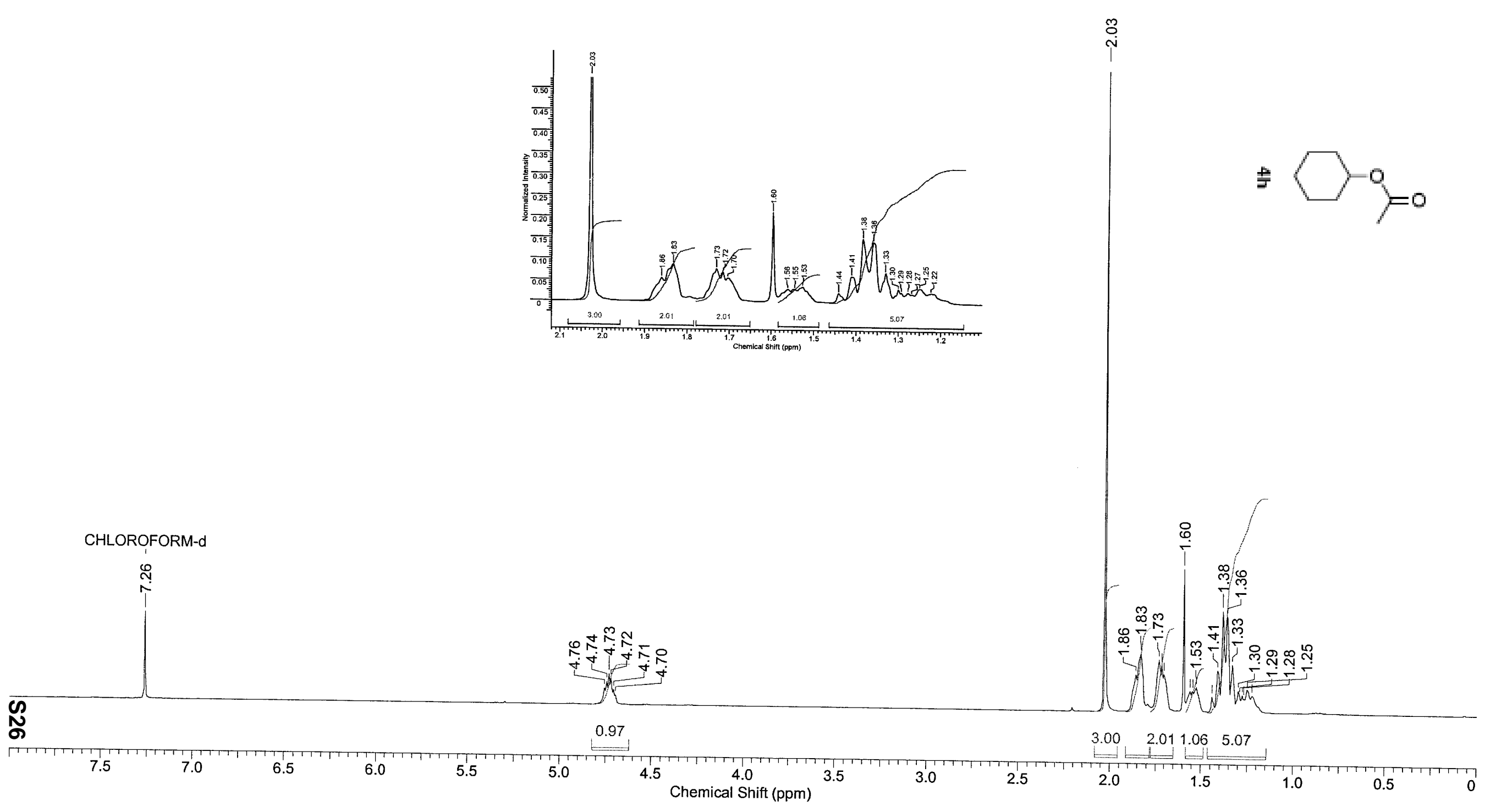


jld13, C13

\begin{tabular}{|c|c|c|c|c|c|c|c|}
\hline \multirow{5}{*}{$\begin{array}{l}\text { Acquisition Time (sec) } \\
\text { File Name } \\
\text { Nucleus } \\
\text { Owner } \\
\text { SW(cyclical) }(\mathrm{Hz}) \\
\text { Sweep Width }(\mathrm{Hz}) \\
\end{array}$} & \multirow{5}{*}{\multicolumn{2}{|c|}{\begin{tabular}{l|l}
1.5139 & Comment \\
IIHOMEIDebieuxJIMy Documents IChimielD \\
$13 \mathrm{C}$ & Number of Transients \\
nmruser & Points Count \\
21645.02 & Solvent \\
21644.36 & Temperature (degree \\
\end{tabular}}} & \multicolumn{3}{|c|}{ jld13, C13 C13CPD CDCl3 u jld 13} & \multirow{3}{*}{\multicolumn{2}{|c|}{\begin{tabular}{|ll} 
Date & 18 Aug 2005 22:04:48 \\
Frequency $(\mathrm{MHz})$ & 90.55 \\
Original Points Count & 32768 \\
\end{tabular}}} \\
\hline & & & ctoratiNn & $13 \_001001 r$ & & & \\
\hline & & & 4096 & Origin & $\mathrm{dp} \times 360$ & & \\
\hline & & & 32768 & Pulse Sequence & zgpg30 & Receiver Gain & 2896.30 \\
\hline & & & CHLOF & & & Spectrum Offset ( $\mathrm{Hz})$ & 9015.4756 \\
\hline
\end{tabular}

CHLOROFORM-d
27.000
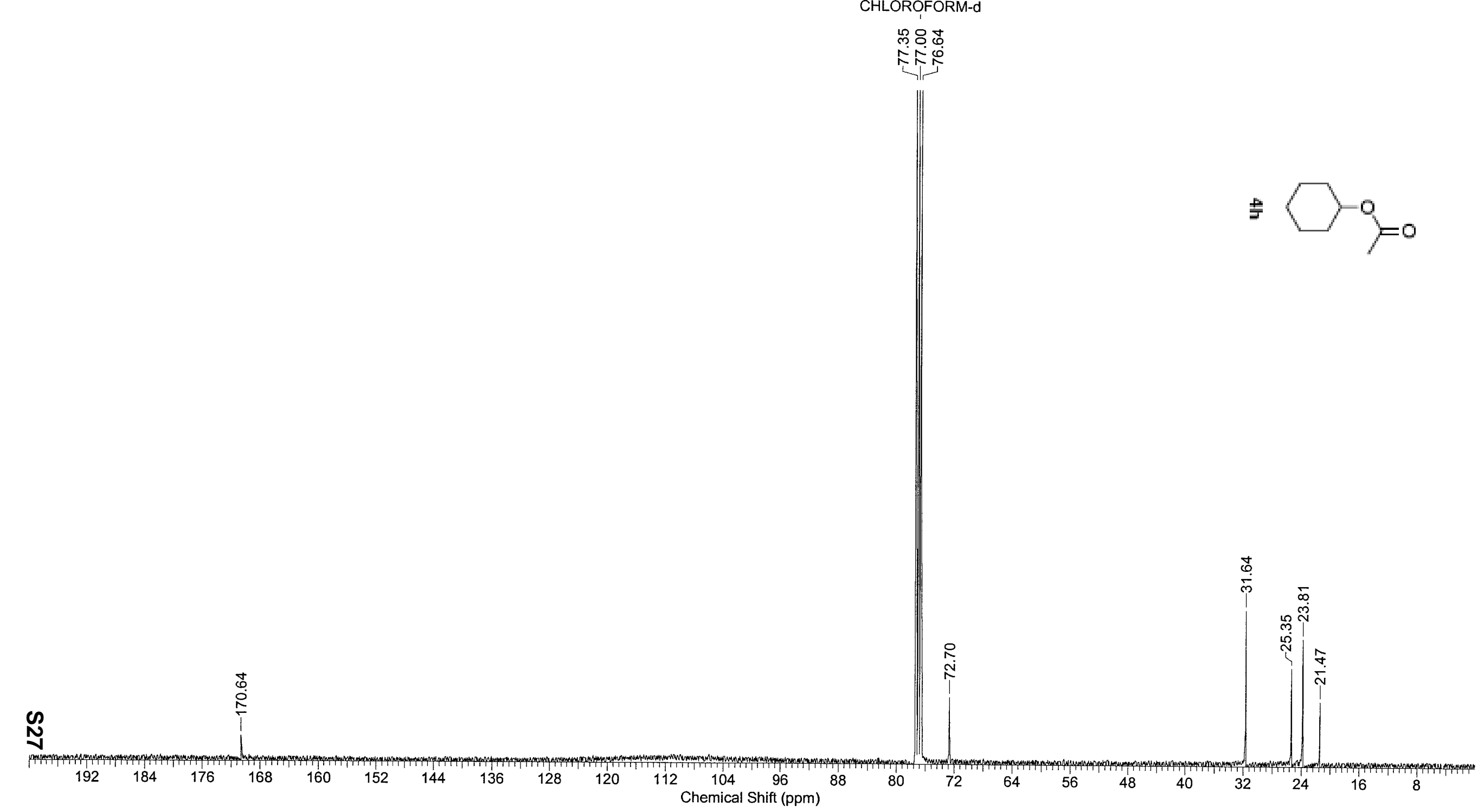


\section{jld14}

\begin{tabular}{|c|c|c|c|c|c|c|c|}
\hline Acquisition Time (sec) & 2.2021 & Comment & \multirow{2}{*}{\multicolumn{3}{|c|}{ jld14, pur PROTONNR $\mathrm{CDCl} 3$ u jld 12}} & Date & 19 Aug 2005 12:43:44 \\
\hline File Name & \multicolumn{4}{|c|}{ IIHOMEIDebieuxJIMy Documents IChimielDoctoratINMRIjld14-1p_001001r } & & Frequency $(\mathrm{MHz})$ & 360.13 \\
\hline Nucleus & $1 \mathrm{H}$ & Number of Transients & 32 & Origin & $\mathrm{dp} \times 360$ & Original Points Count & 16384 \\
\hline Owner & nmruser & Points Count & 16384 & Pulse Sequence & $\mathrm{zg} 30$ & Receiver Gain & 114.00 \\
\hline $\begin{array}{l}\text { SW(cyclical) }(\mathrm{Hz}) \\
\text { Sweep Width }(\mathrm{Hz})\end{array}$ & $\begin{array}{l}7440.48 \\
7440.02\end{array}$ & $\begin{array}{l}\text { Solvent } \\
\text { Temperature (degree C }\end{array}$ & $\frac{\mathrm{CHLOF}}{27.000}$ & & & Spectrum Offset $(\mathrm{Hz})$ & 2210.5947 \\
\hline
\end{tabular}

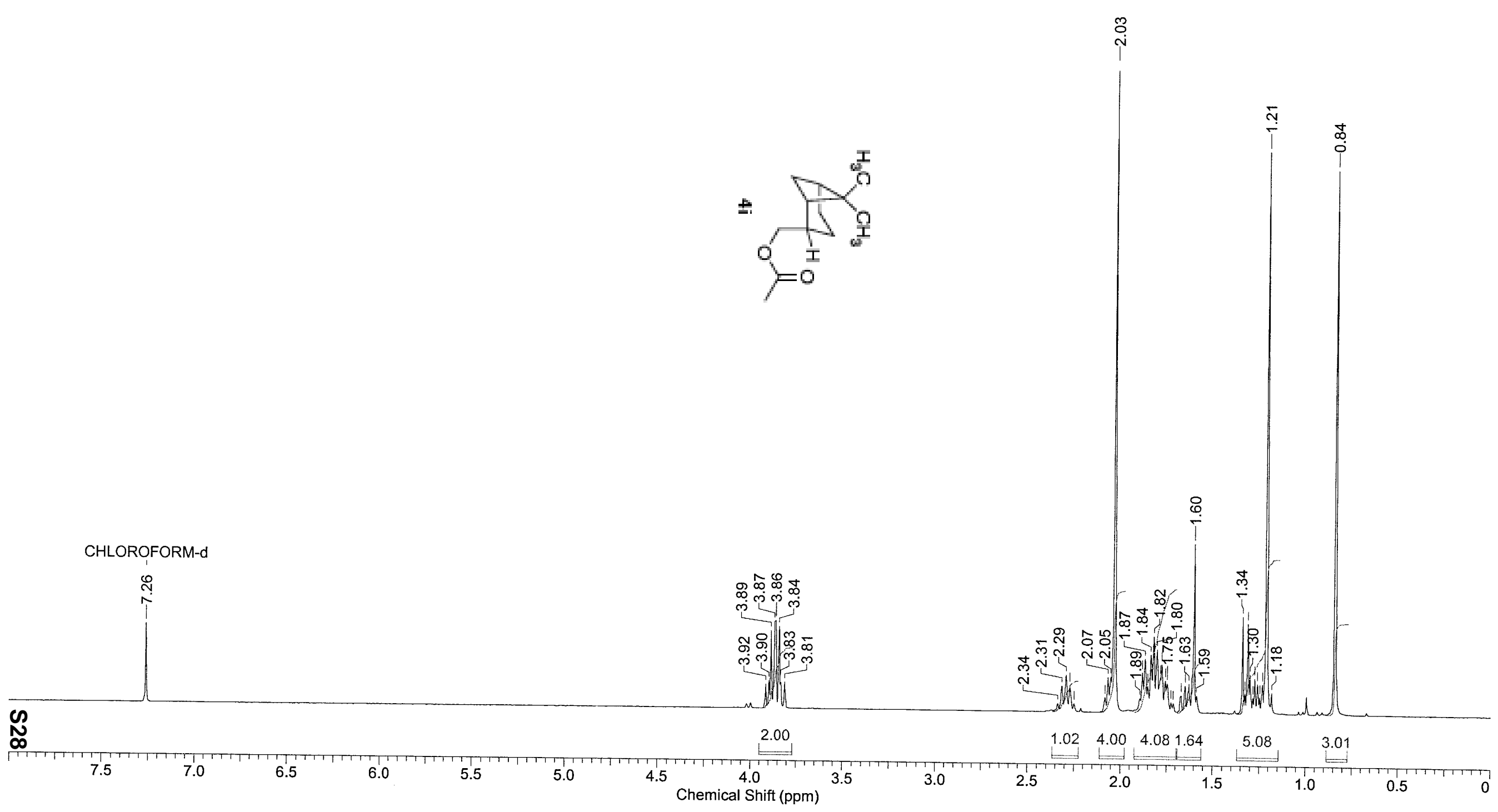


jld14, C13

\begin{tabular}{|c|c|c|c|c|c|c|c|}
\hline Acquisition Time (sec) & 1.5139 & Comment & \multicolumn{3}{|c|}{ jld14_C13 C13CPD CDCl3 u jld 14} & Date & 19 Aug 2005 23:13:04 \\
\hline File Name & \multicolumn{5}{|c|}{ \|HOMEIDebieuxJIMy DocumentsIChimielDoctoratINMRljld14_C13_001001r } & Frequency $(\mathrm{MHz})$ & 90.55 \\
\hline Nucleus & $13 \mathrm{C}$ & Number of Transients & 3072 & Origin & $\mathrm{dp} \times 360$ & Original Points Count & 32768 \\
\hline Owner & nmruser & Points Count & 32768 & Pulse Sequence & zgpg30 & Receiver Gain & 2580.30 \\
\hline $\begin{array}{l}\text { SW(cyclical) }(\mathrm{Hz}) \\
\text { Sweep Width }(H z)\end{array}$ & $\frac{21645.02}{21644.36}$ & Solvent & \multicolumn{3}{|c|}{ CHLOROFORM-d } & Spectrum Offset $(\mathrm{Hz})$ & 9015.4756 \\
\hline
\end{tabular}

\section{CHLOROFORM-d}
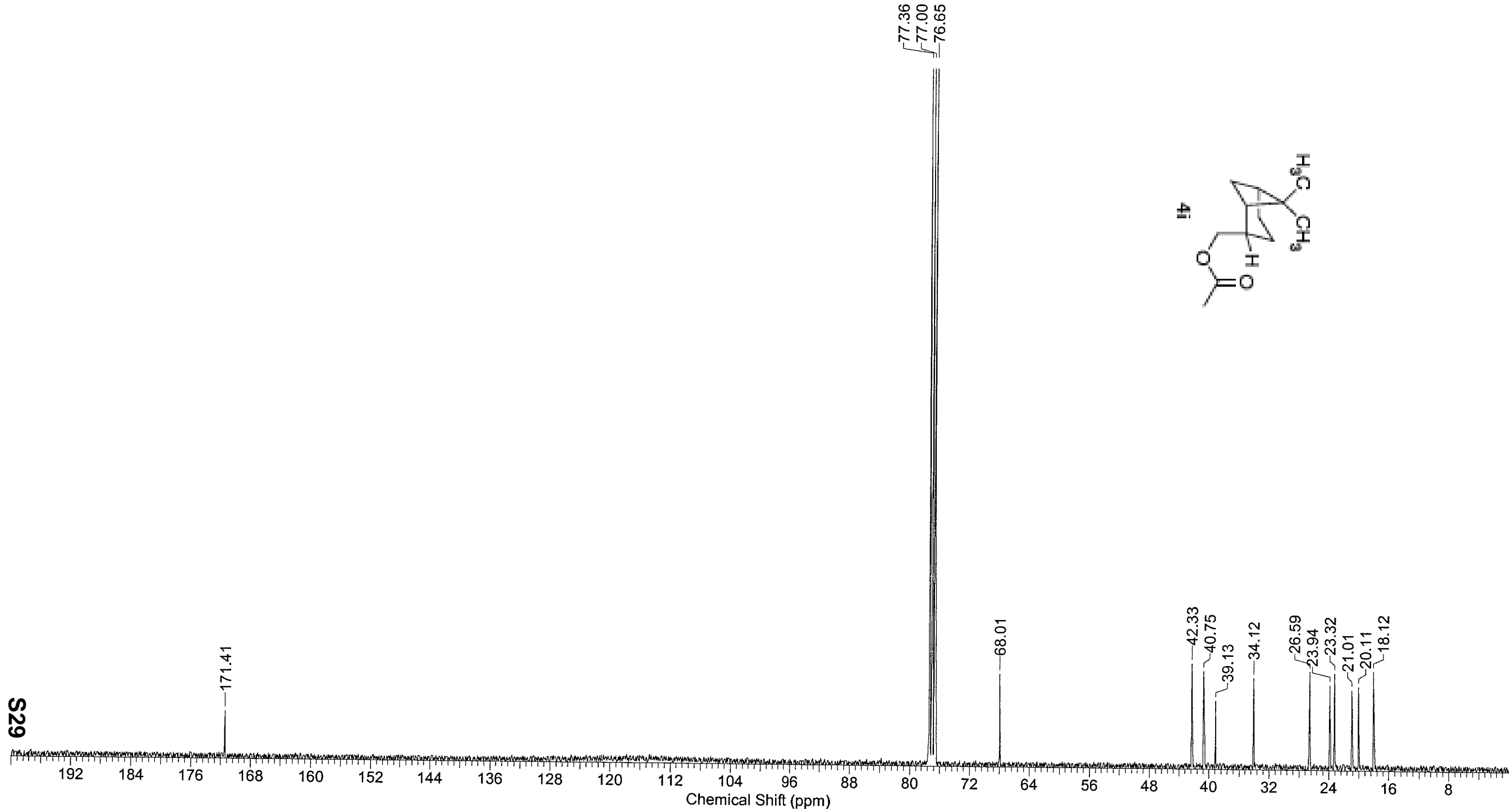


\section{jld15-1}

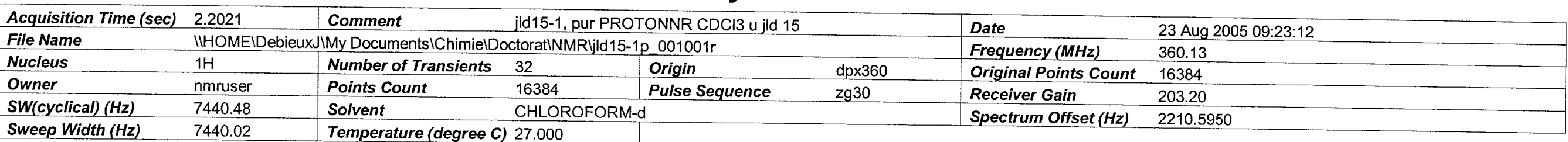

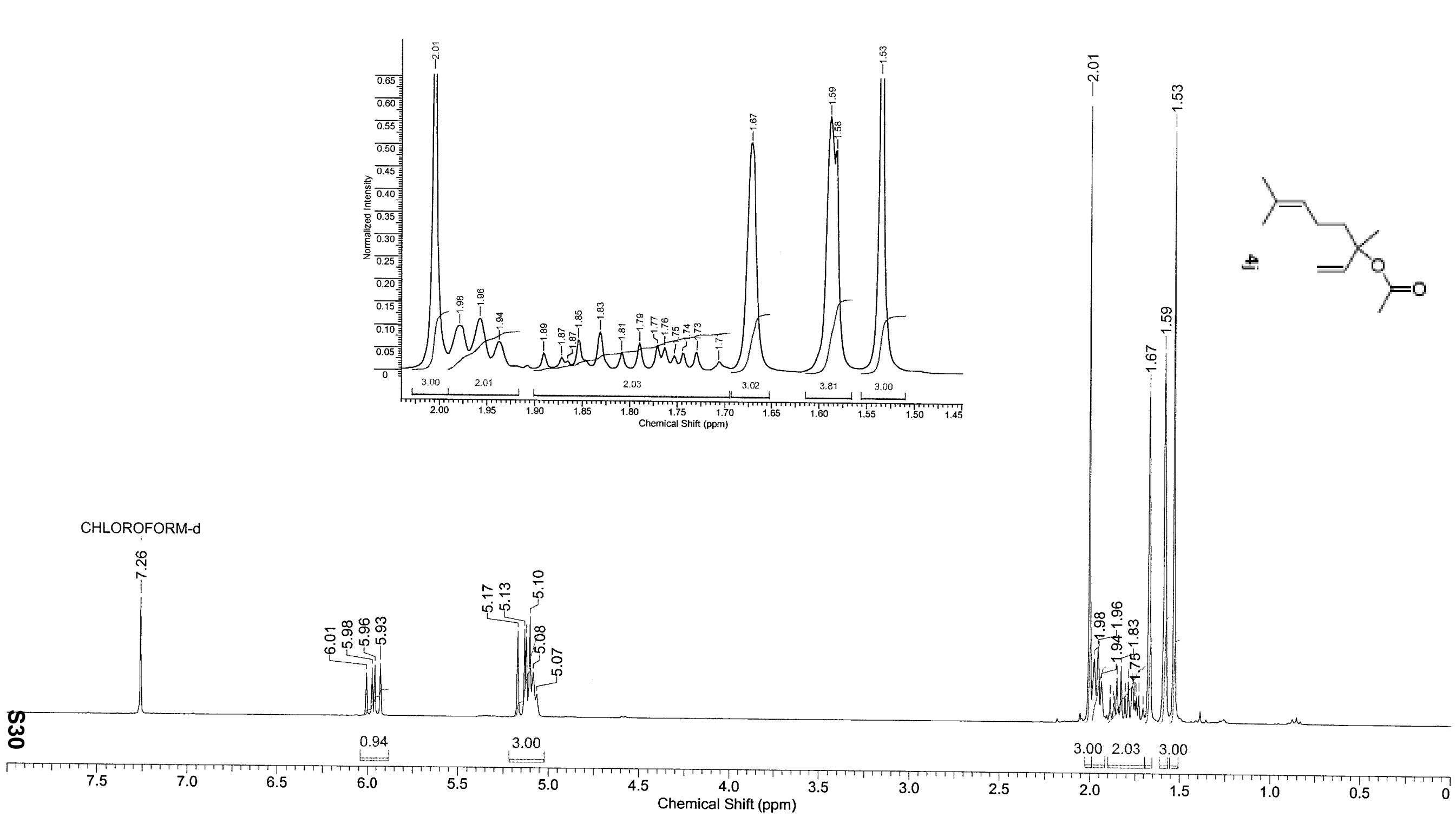




\section{jld15, C13}

\begin{tabular}{|c|c|c|c|c|c|c|c|}
\hline Acquisition Time (sec) & 1.5139 & Comment & \multicolumn{3}{|c|}{ jld15, C13 C13CPD CDCl3 u jld 15} & Date & 25 Aug 2005 02:12:16 \\
\hline File Name & \multicolumn{5}{|c|}{ IIHOMEIDebieuxJIMy DocumentsIChimielDoctoratINMRIjld15_C13_001001r } & Frequency $(\mathrm{MHz})$ & 90.55 \\
\hline Nucleus & $13 \mathrm{C}$ & Number of Transients & 4096 & Origin & $\mathrm{dp} \times 360$ & Original Points Count & 32768 \\
\hline Owner & nmruser & Points Count & 32768 & Pulse Sequence & zgpg30 & Receiver Gain & 3251.00 \\
\hline $\begin{array}{l}\text { SW(cyclical) }(\mathrm{Hz}) \\
\text { Sweep Width }(\mathrm{Hz})\end{array}$ & $\begin{array}{l}21645.02 \\
21644.36\end{array}$ & Solvent & CHLOF & & & Spectrum Offset $(\mathrm{Hz})$ & 9015.4756 \\
\hline
\end{tabular}

\begin{tabular}{ll|l} 
Sweep Width $(\mathrm{Hz})$ & 21644.36 & Temperature (degree C) 27.000
\end{tabular}

CHLOROFORM-d
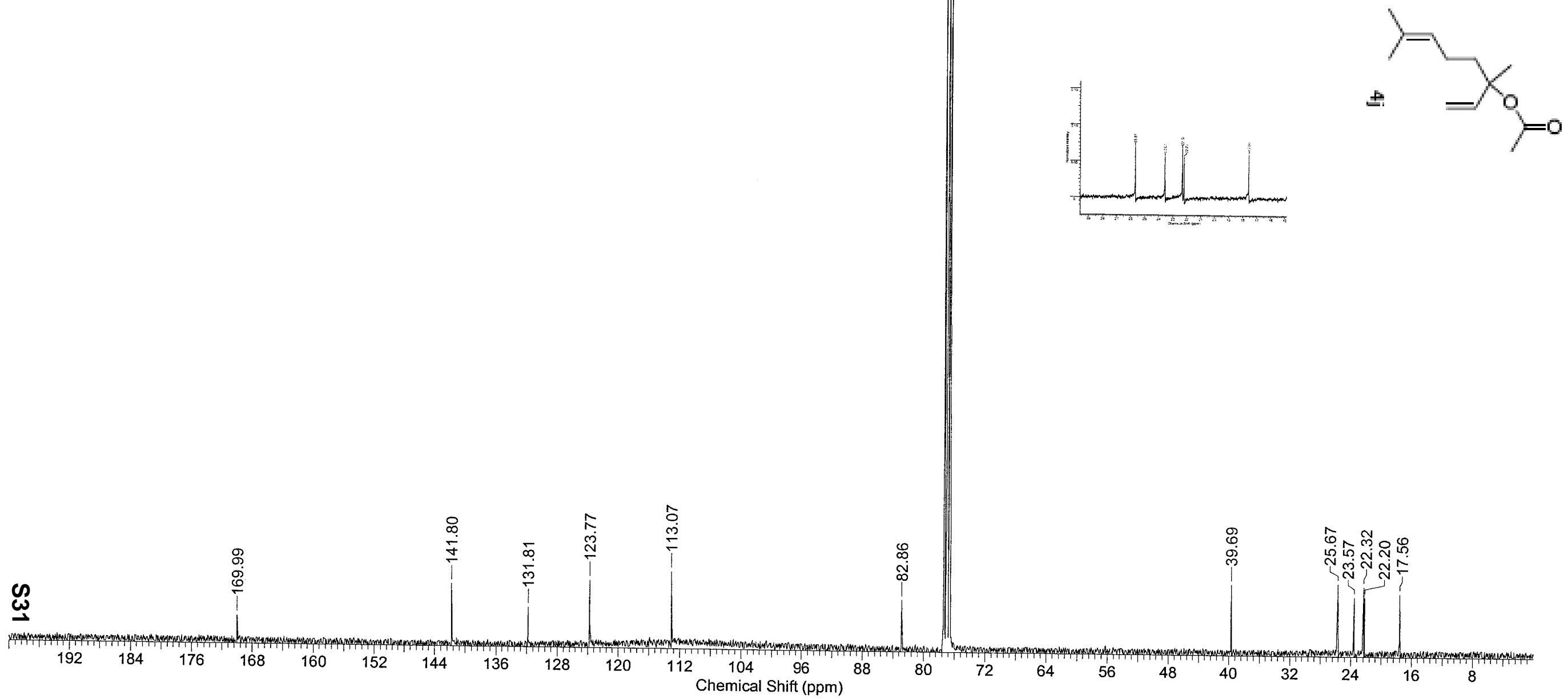


\section{jld16}

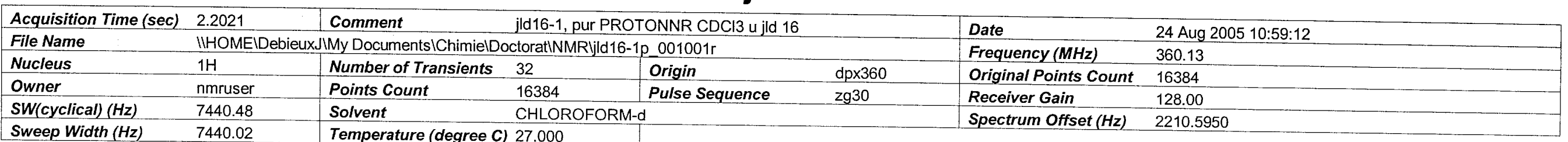

\begin{tabular}{|ll|l|}
\hline Sweep Width $(\mathrm{Hz})$ & 7440.02 & Temperature (degree C) 27.000
\end{tabular}

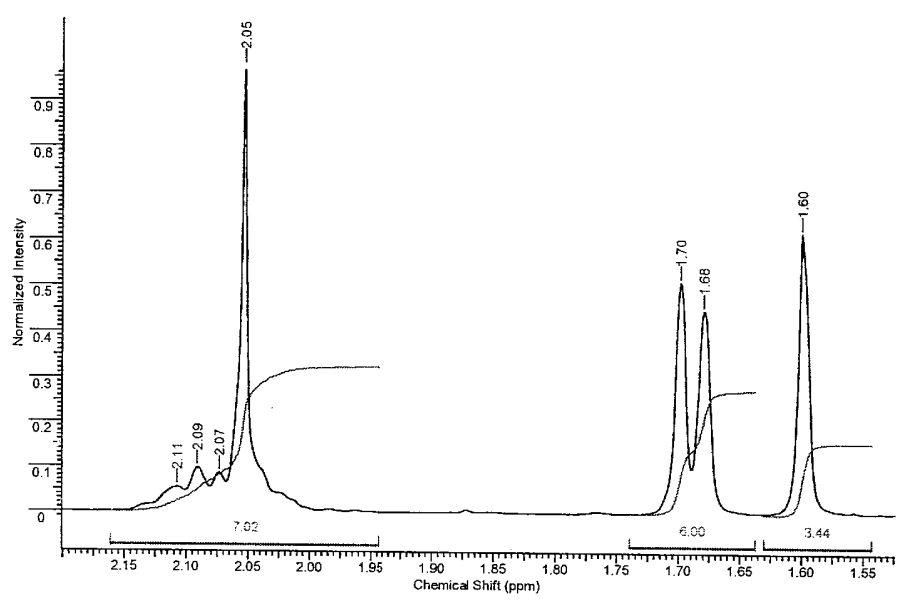

$\stackrel{\infty}{i}$
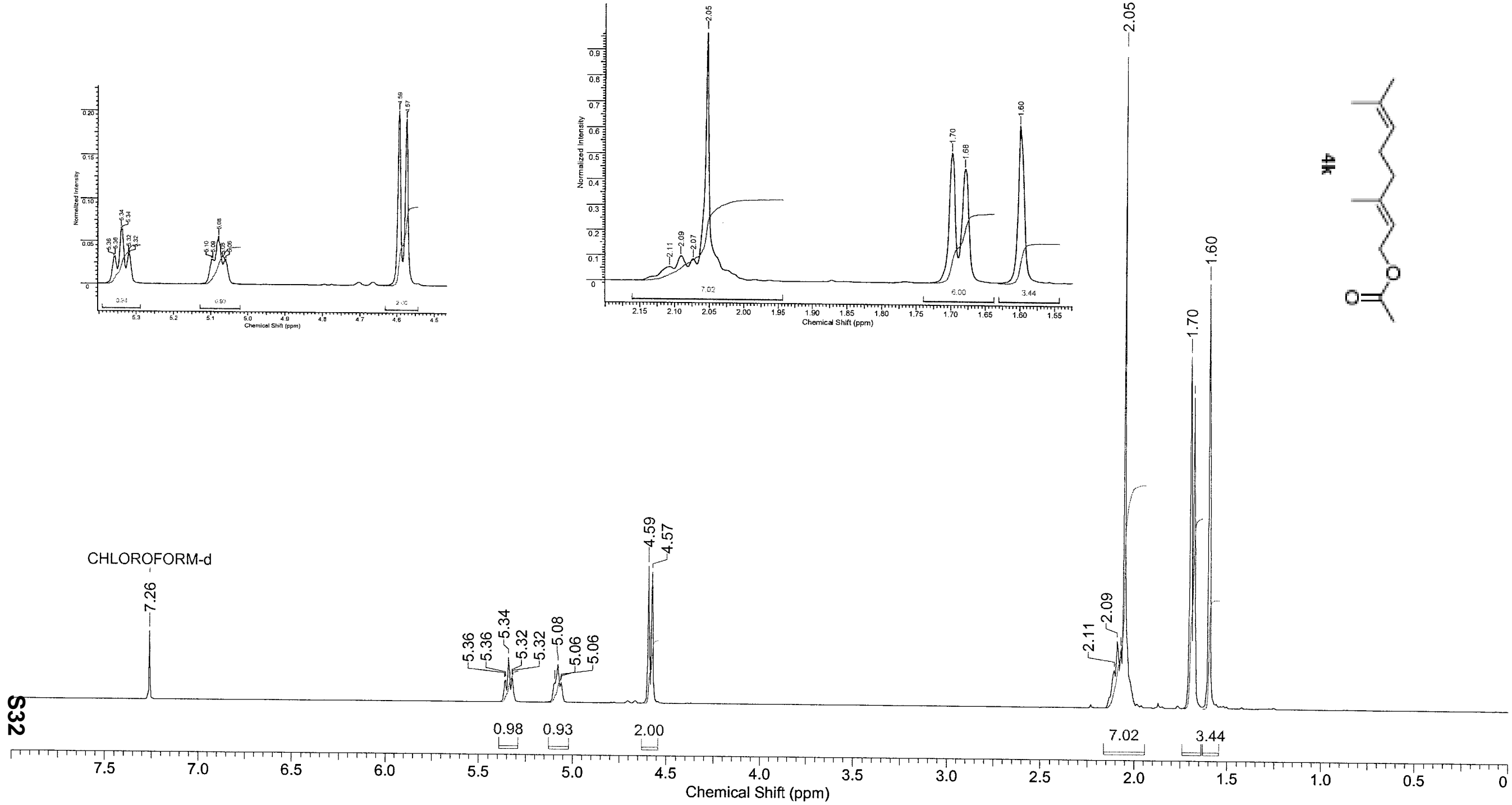


\section{jld16, C13}

\begin{tabular}{|c|c|c|c|c|c|c|c|}
\hline Acquisition Time (sec) & 1.5139 & Comment & \multirow{2}{*}{\multicolumn{3}{|c|}{ jld16, C13 C13CPD CDCl3 u jld 15}} & Date & 25 Aug 2005 22:04:48 \\
\hline File Name & \multicolumn{4}{|c|}{\begin{tabular}{l|l} 
IIHOMEIDebieuxJIMy Documents IChimielDoctoratINMRlild16-1 C13_001001r \\
$13 \mathrm{C}$
\end{tabular}} & & Frequency $(\mathrm{MHz})$ & 90.55 \\
\hline Nucleus & $13 \mathrm{C}$ & Number of Transients & 4096 & Origin & $\mathrm{dp} \times 360$ & Original Points Count & 32768 \\
\hline Owner & nmruser & Points Count & 32768 & Pulse Sequence & zgpg30 & Receiver Gain & 4096.00 \\
\hline SW(cyclical) $(\mathrm{Hz})$ & 21645.02 & Solvent & CHLOR & & & Spectrum Offset $(\mathrm{Hz})$ & 9015.4766 \\
\hline
\end{tabular}

Sweep Width $(\mathrm{Hz}) \quad 21644.36$ Temperature (degree C) 27.000

CHLOROFORM-d

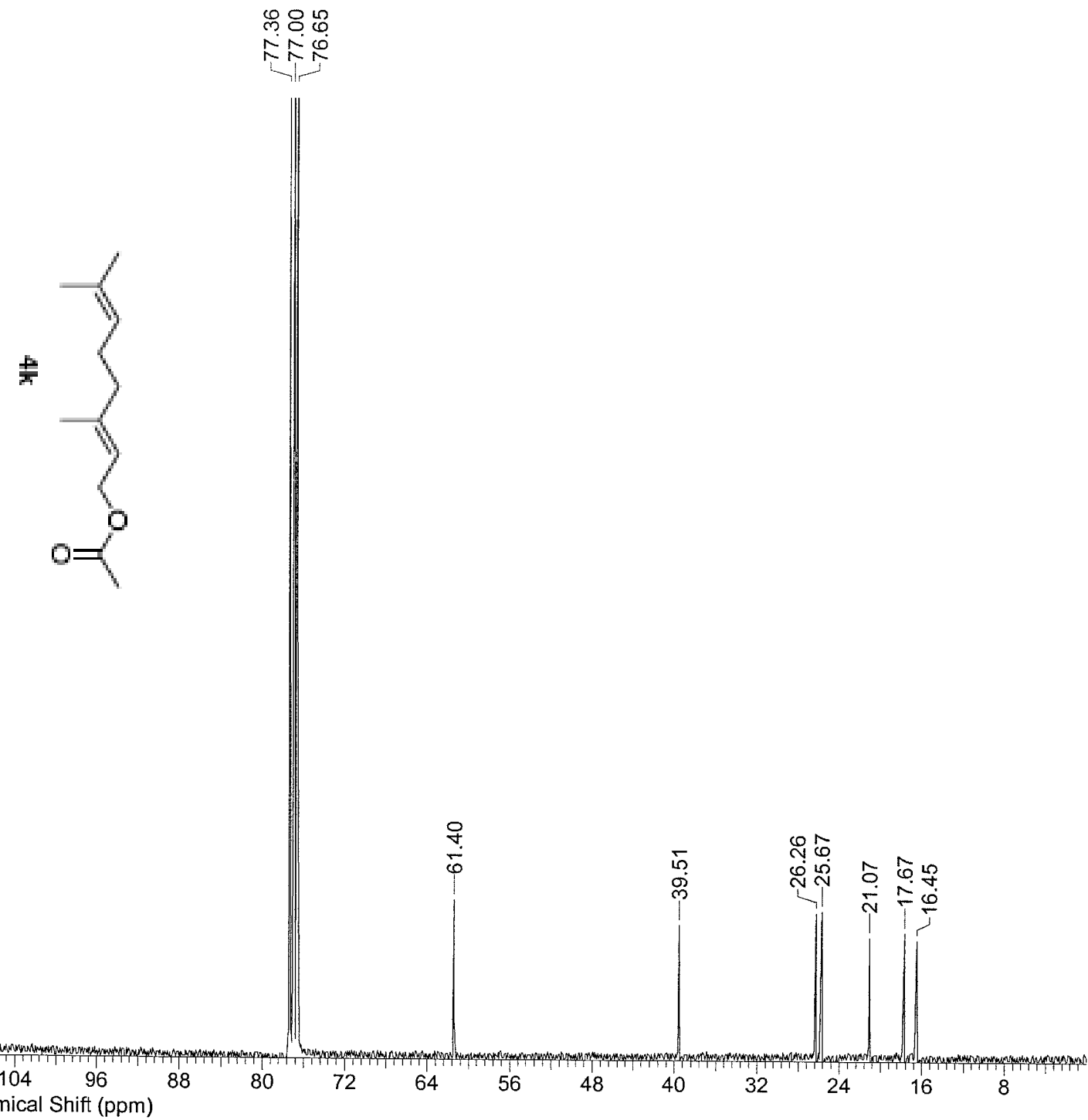




\section{jld17}

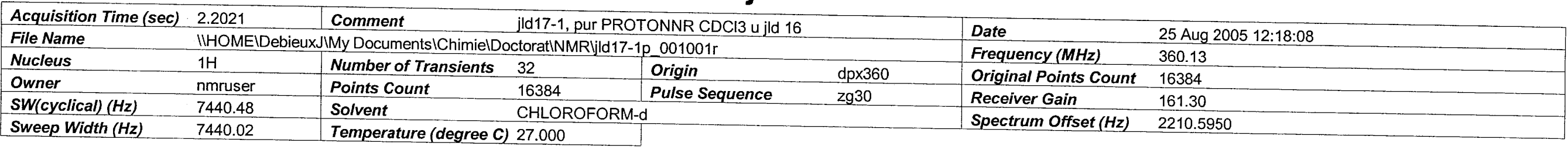

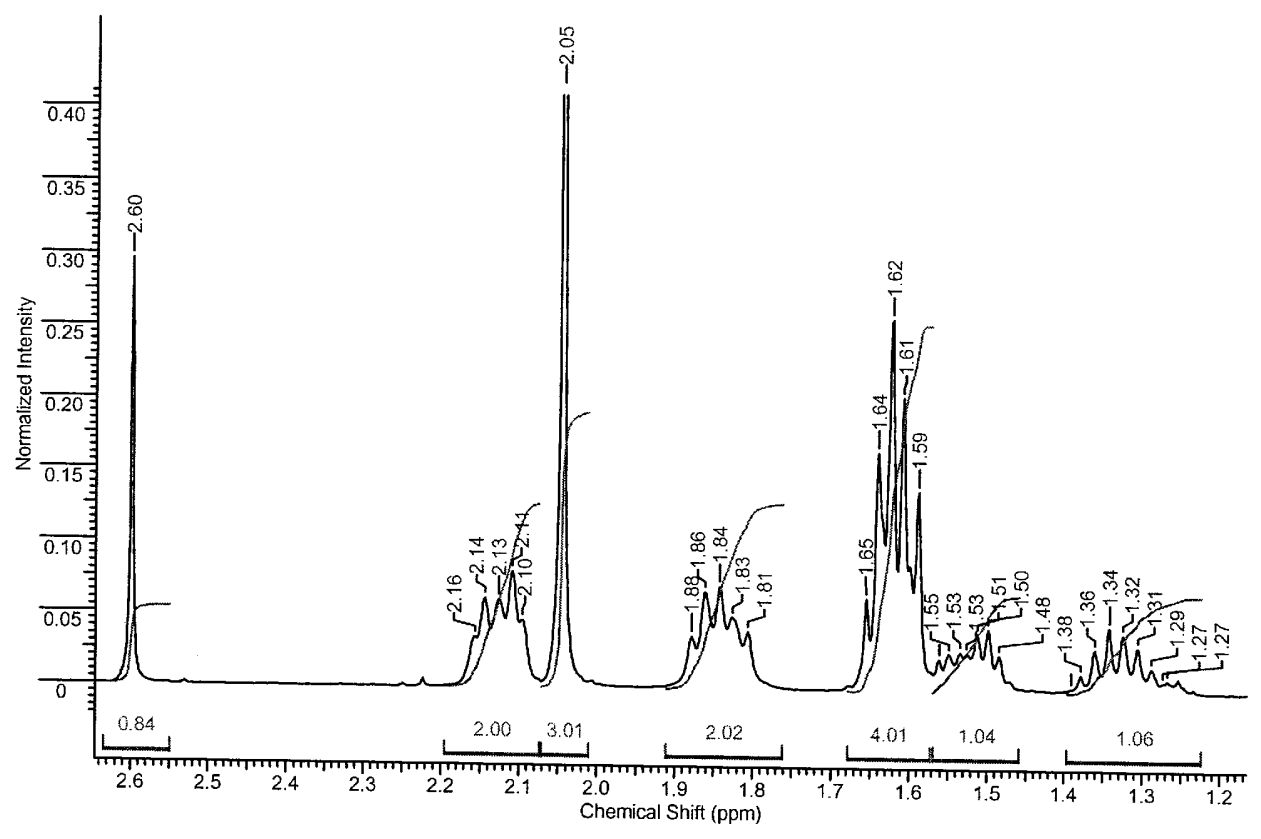

CHLOROFORM-d

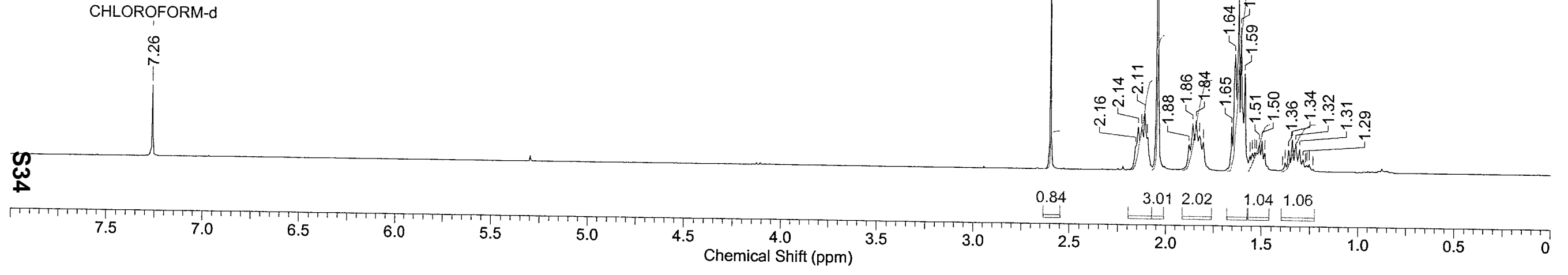


jld17, C13

\begin{tabular}{|c|c|c|c|c|c|c|c|}
\hline Acquisition Time (sec) & 1.5139 & Comment & jld17, C & D CDCl3 u jld 17 & & Date & 26 Aug 2005 04:56:32 \\
\hline File Name & IIHOMEID & My DocumentsiChimielD & ctoratiNn & $13 \quad 001001$ r & & Frequency $(\mathrm{MHz})$ & 90.55 \\
\hline Nucleus & $13 \mathrm{C}$ & Number of Transients & 4096 & Origin & $\mathrm{dp} \times 360$ & Original Points Count & 32768 \\
\hline Owner & nmruser & Points Count & 32768 & Pulse Sequence & zgpg30 & Receiver Gain & 1824.60 \\
\hline $\begin{array}{l}\text { SW(cyclical) }(\mathrm{Hz}) \\
\text { Sweep Width }(\mathrm{Hz})\end{array}$ & $\frac{21645.02}{21644.36}$ & Solvent & $\mathrm{CHLOR}$ & & & Spectrum Offset $(\mathrm{Hz})$ & 9015.4756 \\
\hline
\end{tabular}

$\begin{array}{llll}\text { Sweep Width }(\mathrm{Hz}) & 21644.36 & \text { Temperature (degree C) } 27.000\end{array}$

\section{CHLOROFORM-d}

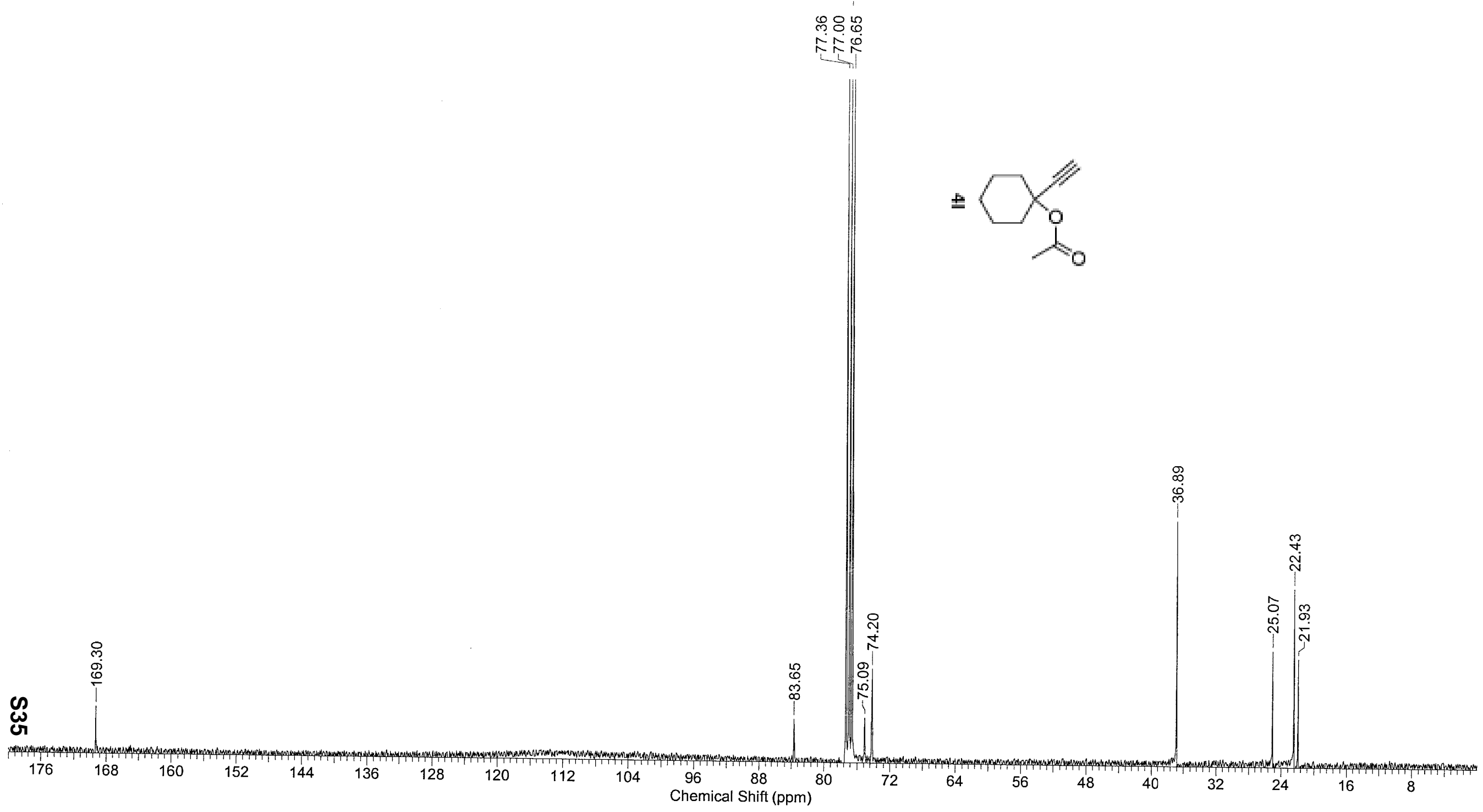


jld19

\begin{tabular}{|c|c|c|c|c|c|c|c|}
\hline Acquisition Time (sec) & 2.2021 & Comment & \multirow{2}{*}{\multicolumn{3}{|c|}{ jld19-2, pur PROTONNR CDCl3 u jld 19}} & Date & $02 \operatorname{Sep} 2005$ 15:30:08 \\
\hline File Name & \multicolumn{3}{|c|}{ IIHOMEIDebieuxJIMy DocumentsiChimielDoctoratINMRlild19-2p2_001001r } & & & Frequency $(\mathrm{MHz})$ & 360.13 \\
\hline Nucleus & $1 \mathrm{H}$ & Number of Transients & 16 & Origin & $\mathrm{dp} \times 360$ & \multicolumn{2}{|c|}{ Original Points Count 16384} \\
\hline Owner & nmruser & Points Count & 16384 & Pulse Sequence & $\mathrm{zg} 30$ & Receiver Gain & 203.20 \\
\hline SW(cyclical) $(\mathrm{Hz})$ & 7440.48 & Solvent & \multicolumn{3}{|c|}{ CHLOROFORM-d } & \multicolumn{2}{|c|}{ Spectrum Offset $(\mathrm{Hz}) \quad 2210.5947$} \\
\hline
\end{tabular}

Sweep Width $(\mathrm{Hz}) \quad 7440.02$

Temperature (degree C) 27.000

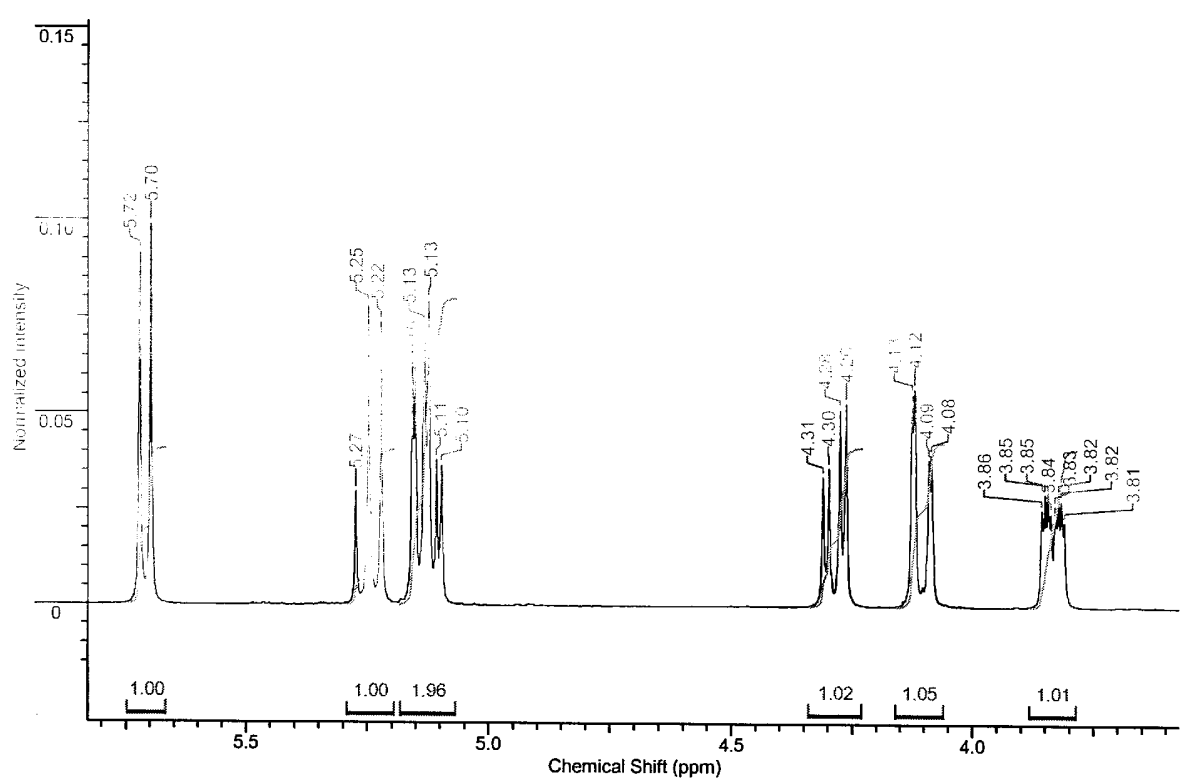

CHLOROFORM-d

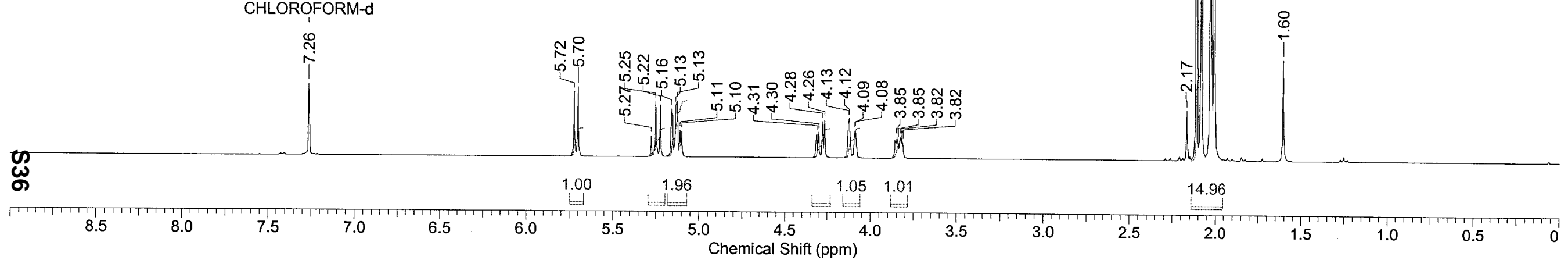




\section{jld19, C13}

\begin{tabular}{|c|c|c|c|c|c|c|}
\hline Acquisition Time (sec) & 1.5139 & Comment & \multirow{2}{*}{\multicolumn{2}{|c|}{ jld19, C13 C13CPD CDCl3 u jld 19}} & Date & 03 Sep 2005 08:14:56 \\
\hline File Name & \multicolumn{3}{|c|}{\begin{tabular}{l|l|l} 
IHOMEIDebieux.JMy Documents IChimielDoctoratINMRlild19_C13_001001r \\
$13 \mathrm{C}$ & Number of Transients
\end{tabular}} & & Frequency $(\mathrm{MHz})$ & 90.55 \\
\hline Nucleus & $13 \mathrm{C}$ & Number of Transients & 2048 & Origin & Original Points Count & 32768 \\
\hline Owner & nmruser & Points Count & 32768 & Pulse Sequence & Receiver Gain & 2580.30 \\
\hline $\begin{array}{l}\text { SW(cyclical) }(\mathrm{Hz}) \\
\text { Sweep Width }(\mathrm{Hz})\end{array}$ & $\frac{21645.02}{21644.36}$ & Solvent & \multicolumn{2}{|c|}{ CHLOROFORM-d } & Spectrum Offset $(\mathrm{Hz})$ & 9014.8154 \\
\hline
\end{tabular}

CHLOROFORM-d

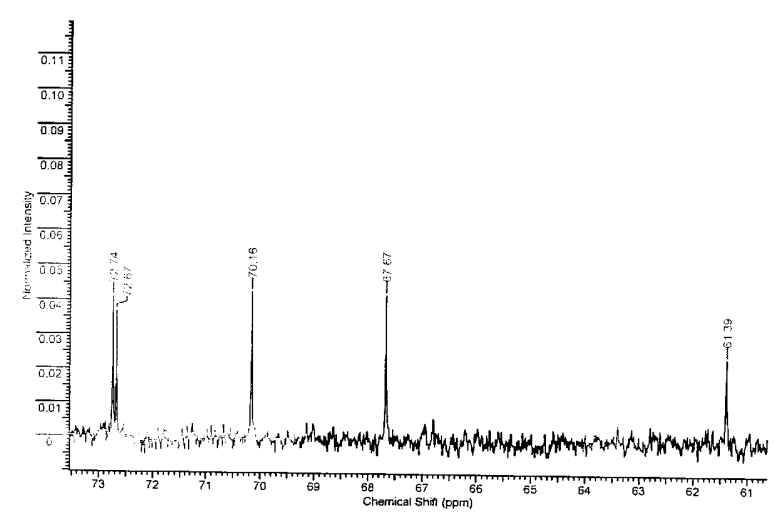

๓̊ 8 ษ

全令

\section{III}
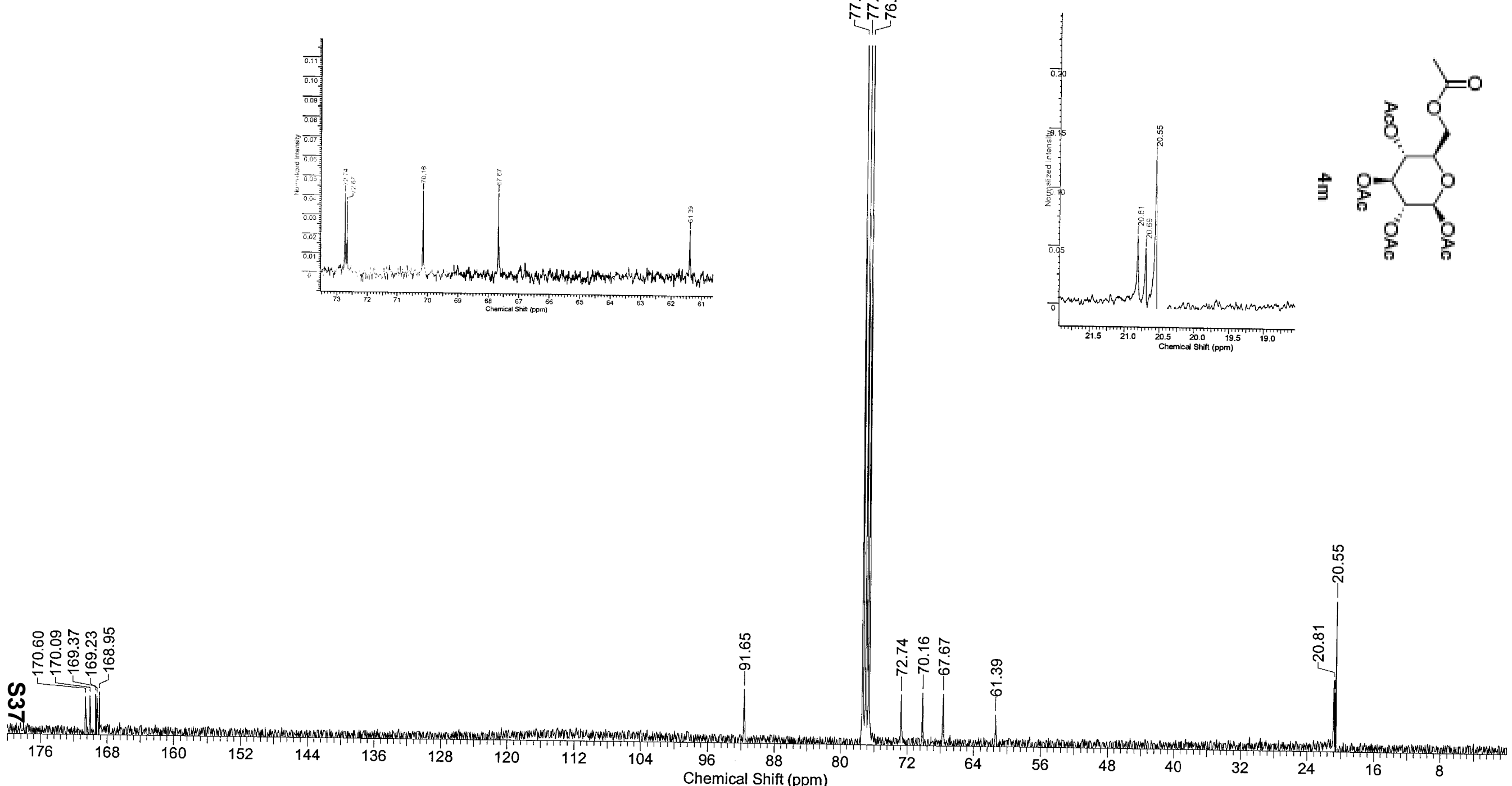


\section{jld20}

\begin{tabular}{|c|c|c|c|c|c|c|c|}
\hline Acquisition Time (sec) & 2.2021 & Comment & \multicolumn{3}{|c|}{ jld20-1, pur PROTONNR CDCl3 u jld 20} & Date & 01 Sep 2005 09:44:32 \\
\hline File Name & \multicolumn{5}{|c|}{ IIHOMEIDebieuxJIMy DocumentsIChimielDoctoratINMRljld20-1p_001001r } & Frequency $(\mathrm{MHz})$ & 360.13 \\
\hline Nucleus & $1 \mathrm{H}$ & Number of Transients & 32 & Origin & $\mathrm{dp} \times 360$ & Original Points Count & 16384 \\
\hline Owner & nmruser & Points Count & 16384 & Pulse Sequence & $\mathrm{zg} 30$ & Receiver Gain & 228.10 \\
\hline SW(cyclical) $(\mathrm{Hz})$ & 7440.48 & Solvent & CHLOF & & & Spectrum Offset (Hz) & 2210.5950 \\
\hline
\end{tabular}

Sweep Width $(\mathrm{Hz}) \quad 7440.02$ Temperature (degree C) 27.000

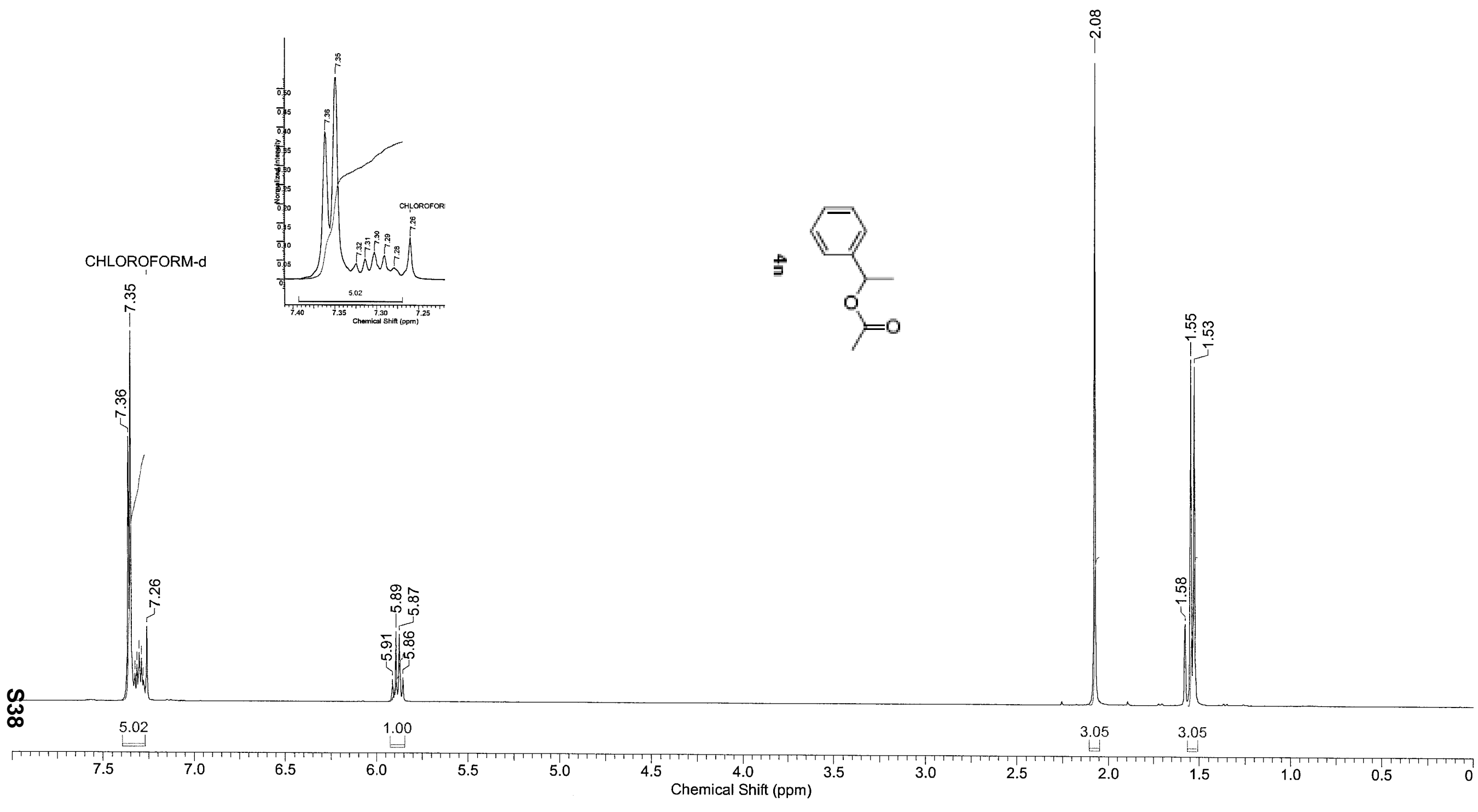




\section{jld20, C13}

\begin{tabular}{|c|c|c|c|c|c|c|c|}
\hline Acquisition Time (sec) & 1.5139 & Comment & \multirow{2}{*}{\multicolumn{3}{|c|}{ jld20, C13 C13CPD CDCl3 u jld 20}} & Date & 01 Sep 2005 22:04:48 \\
\hline File Name & \multicolumn{4}{|c|}{ IHHOMEIDebieuxJIMy DocumentsIChimielDoctoratINMRlild20_C13_001001r } & & Frequency $(\mathrm{MHz})$ & 90.55 \\
\hline Nucleus & $13 \mathrm{C}$ & Number of Transients & 4096 & Origin & $\mathrm{dp} \times 360$ & Original Points Count & 32768 \\
\hline Owner & nmruser & Points Count & 32768 & Pulse Sequence & zgpg30 & Receiver Gain & 1824.60 \\
\hline Sweep Width $(\mathrm{Hz})$ & $\frac{21645.02}{21644.36}$ & $\frac{\text { Solvent }}{\text { Temperature (degree } C}$ & \multicolumn{3}{|c|}{ CHLOROFORM-d } & Spectrum Offset $(\mathrm{Hz})$ & 9015.4756 \\
\hline
\end{tabular}

\begin{tabular}{ll|l|l} 
Sweep Width $(\mathrm{Hz})$ & 21644.36 & Temperature (degree C) 27.000 & CHLOROFORM-d
\end{tabular}

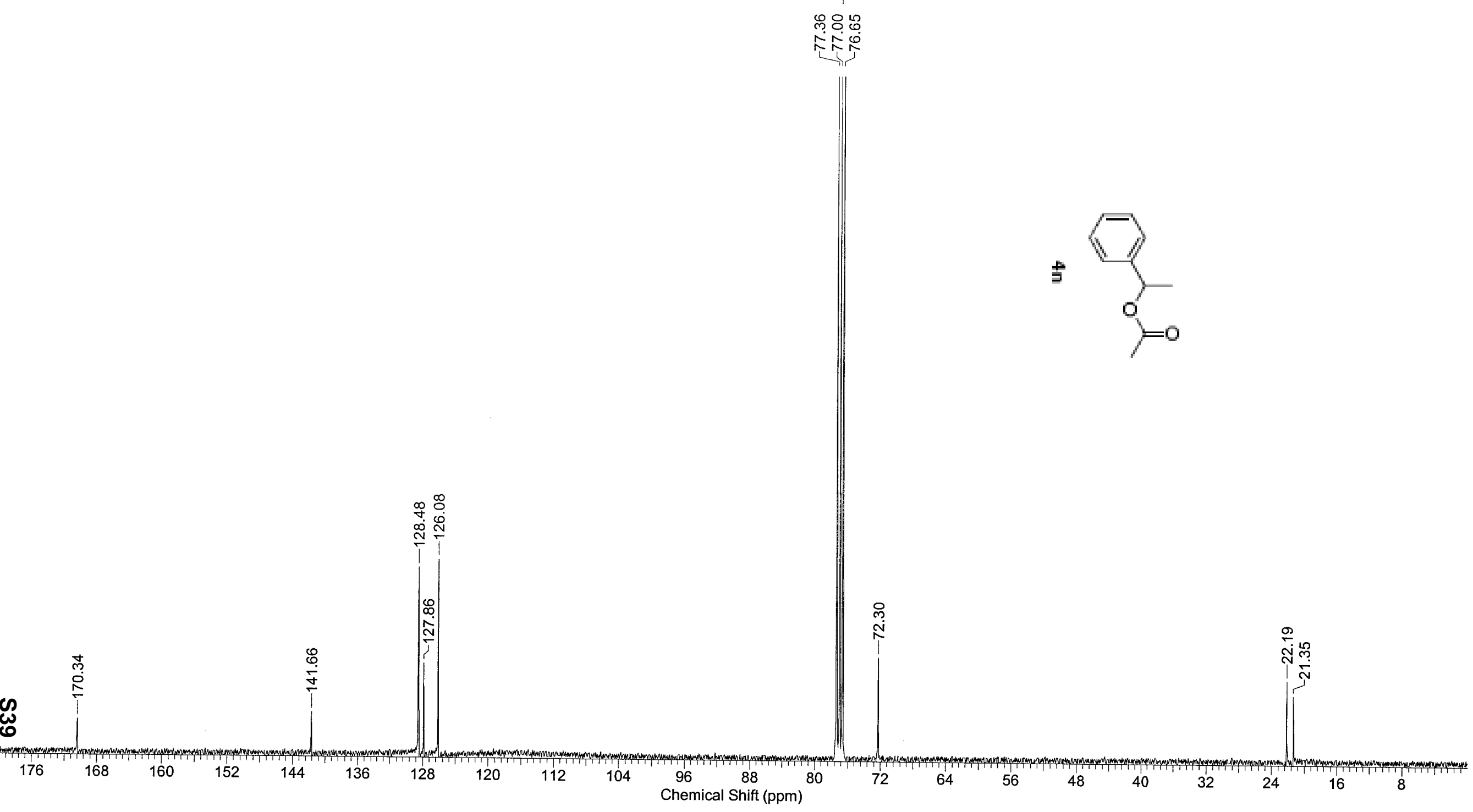




\section{jld21}

\begin{tabular}{|c|c|c|c|c|c|c|c|}
\hline Acquisition Time (sec) & 2.2021 & Comment & jld21-1p & JNR CDCl3 u jld 26 & & Date & $06 \operatorname{Sep} 2005$ 10:44:16 \\
\hline File Name & UHOMEI & IMy DocumentsiChimielD & ctoratiNI & p_001001r & & Frequency $(\mathrm{MHz})$ & 360.13 \\
\hline Nucleus & $1 \mathrm{H}$ & Number of Transients & 32 & Origin & $\mathrm{dp} \times 360$ & Original Points Count & 16384 \\
\hline Owner & nmruser & Points Count & 16384 & Pulse Sequence & $\mathrm{zg} 30$ & Receiver Gain & 203.20 \\
\hline$S W$ (cyclical) $(\mathrm{Hz})$ & 7440.48 & Solvent & $\mathrm{CHLOR}$ & & & Spectrum Offset $(\mathrm{Hz})$ & 2210.5950 \\
\hline
\end{tabular}
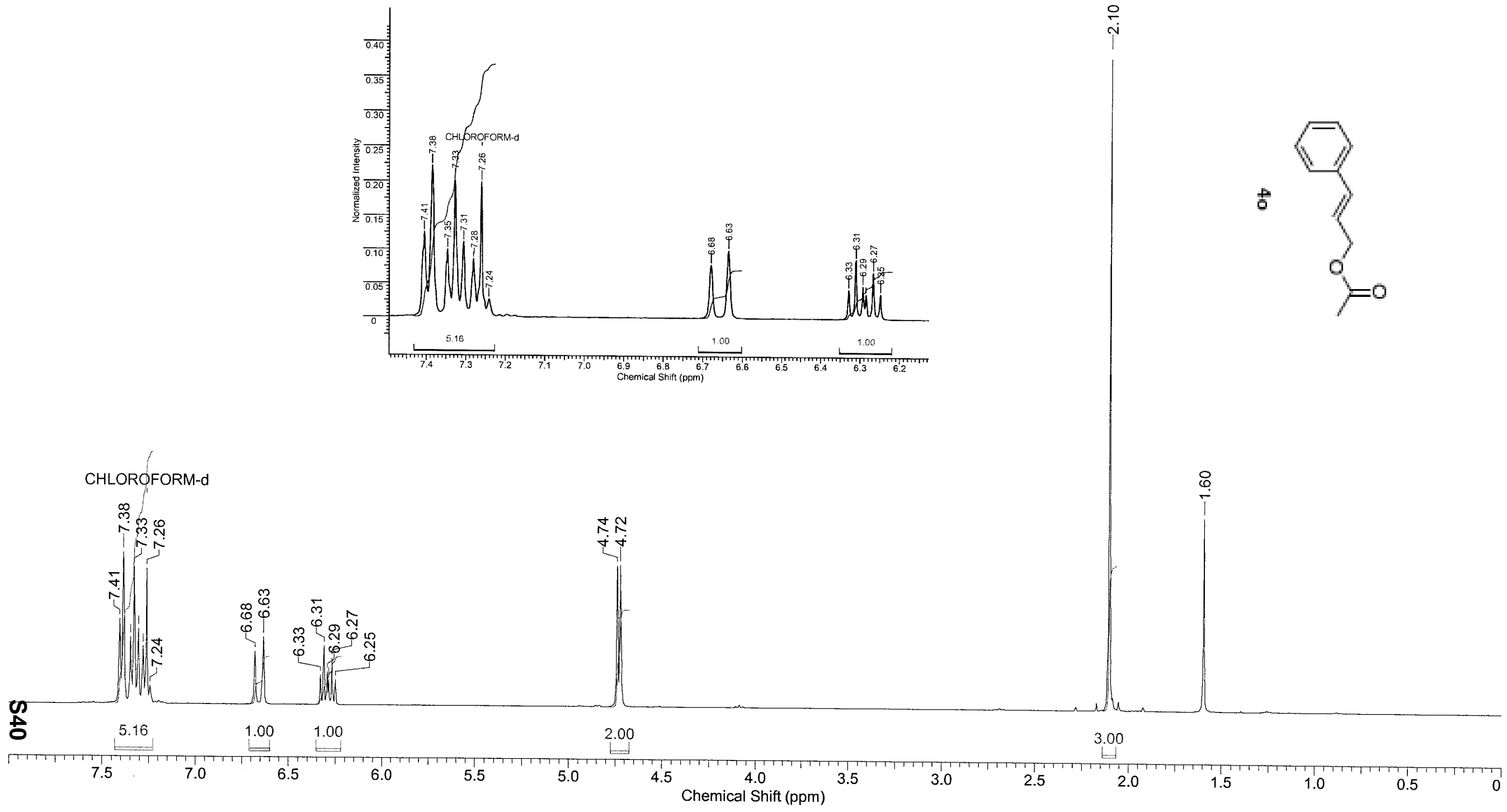
jld21, C13

\begin{tabular}{|c|c|c|c|c|c|c|c|}
\hline Acquisition Time (sec) & 1.5139 & Comment & \multirow{2}{*}{\multicolumn{3}{|c|}{ jld21 C13 C13CPD CDCl3 u jld 26}} & Date & 06 Sep 2005 22:04:48 \\
\hline File Name & \multicolumn{4}{|c|}{ IIHOMEIDebieuxJIMy DocumentsIChimielDoctoratINMRIjld21_C13_001001r } & & Frequency $(\mathrm{MHz})$ & 90.55 \\
\hline Nucleus & $13 \mathrm{C}$ & Number of Transients & 4096 & Origin & $d p \times 360$ & Original Points Count & 32768 \\
\hline Owner & nmruser & Points Count & 32768 & Pulse Sequence & zgpg30 & Receiver Gain & 2580.30 \\
\hline SW(cyclical) $(\mathrm{Hz})$ & 21645.02 & Solvent & CHLOR & & & Spectrum Offset $(\mathrm{Hz})$ & 9014.8154 \\
\hline
\end{tabular}

$\begin{array}{lll}\text { Sweep Width }(\mathrm{Hz}) & 21644.36 & \text { Temperature (degree C) } 27.000\end{array}$

CHLOROFORM-d
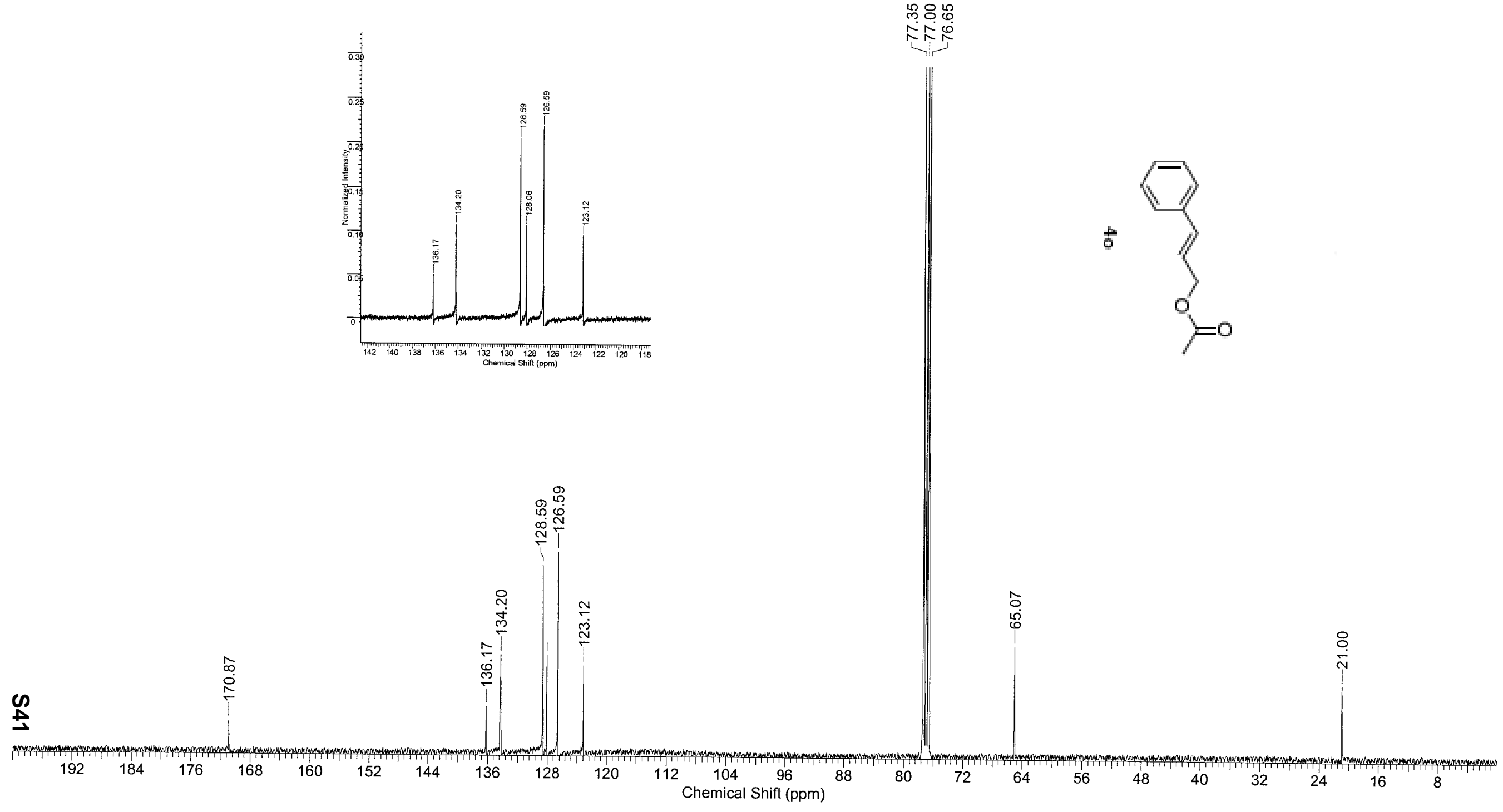


\section{jld22}

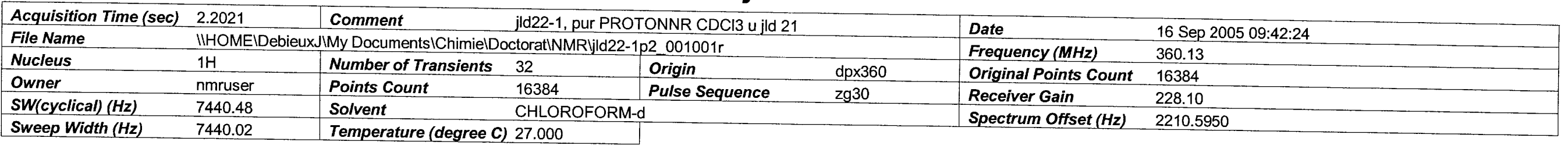
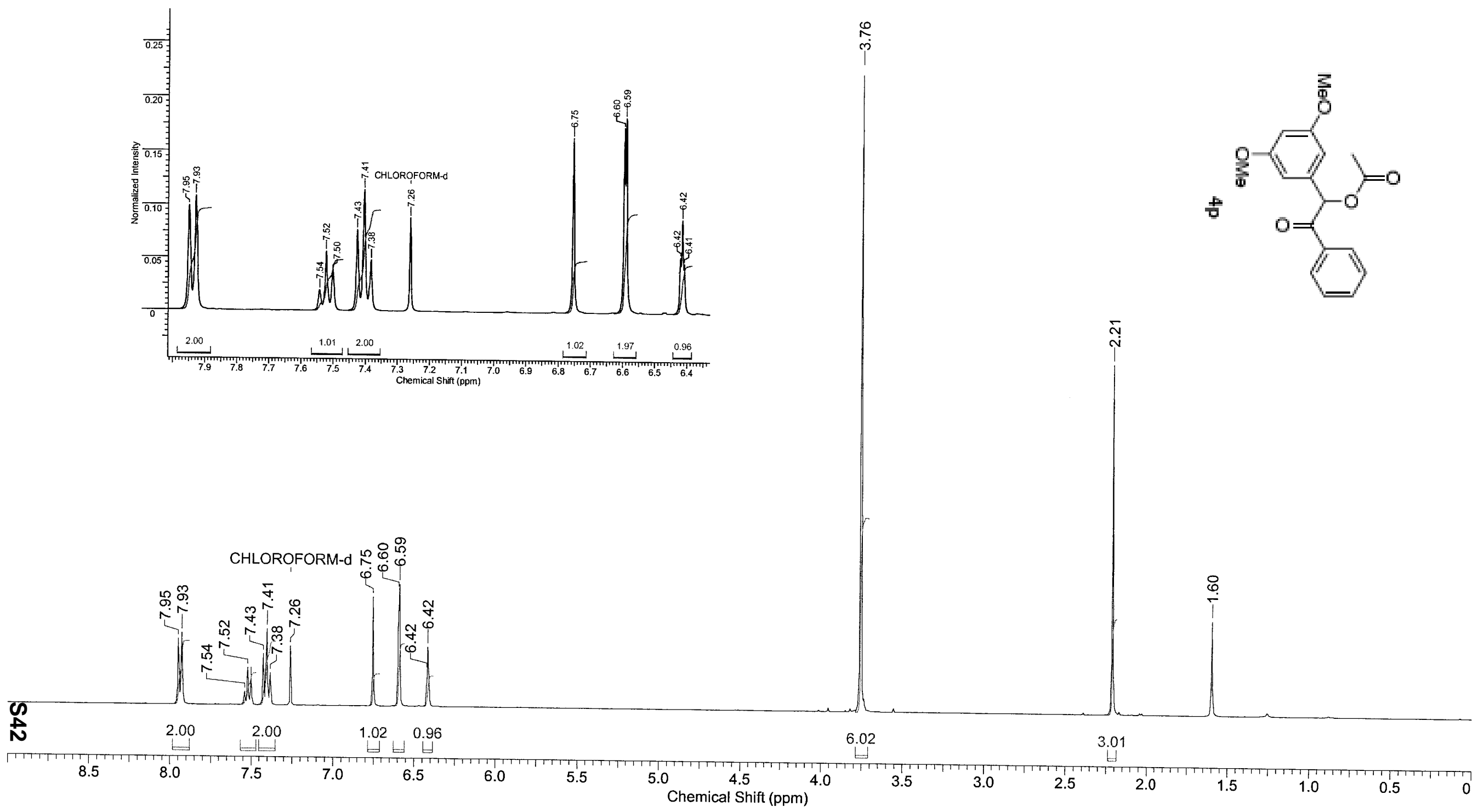
jld22, C13

\begin{tabular}{|c|c|c|c|c|c|c|c|}
\hline Acquisition Time (sec) & 1.5139 & Comment & jld22-1, & CPD CDCl3 u jld 22 & & Date & $16 \operatorname{Sep} 2005$ 22:06:56 \\
\hline File Name & IIHOMEID & My DocumentsiChimielDo & ctoratiNn & $13 \_001001 r$ & & Frequency $(\mathrm{MHz})$ & 90.55 \\
\hline Nucleus & $13 \mathrm{C}$ & Number of Transients & 4096 & Origin & $\mathrm{dp} \times 360$ & Original Points Count & 32768 \\
\hline Owner & nmruser & Points Count & 32768 & Pulse Sequence & zgpg30 & Receiver Gain & 2896.30 \\
\hline $\begin{array}{l}\text { SW(cyclical) }(\mathrm{Hz}) \\
\text { Sweep Width }(\mathrm{Hz})\end{array}$ & $\begin{array}{l}21645.02 \\
21644.36\end{array}$ & Solvent & CHLOF & & & Spectrum Offset $(\mathrm{Hz})$ & 9014.8154 \\
\hline
\end{tabular}

\begin{tabular}{|lll} 
Sweep Width $(\mathrm{Hz})$ & 21644.36 & Temperature (degree C) 27.000
\end{tabular}

CHLOROFORM-d

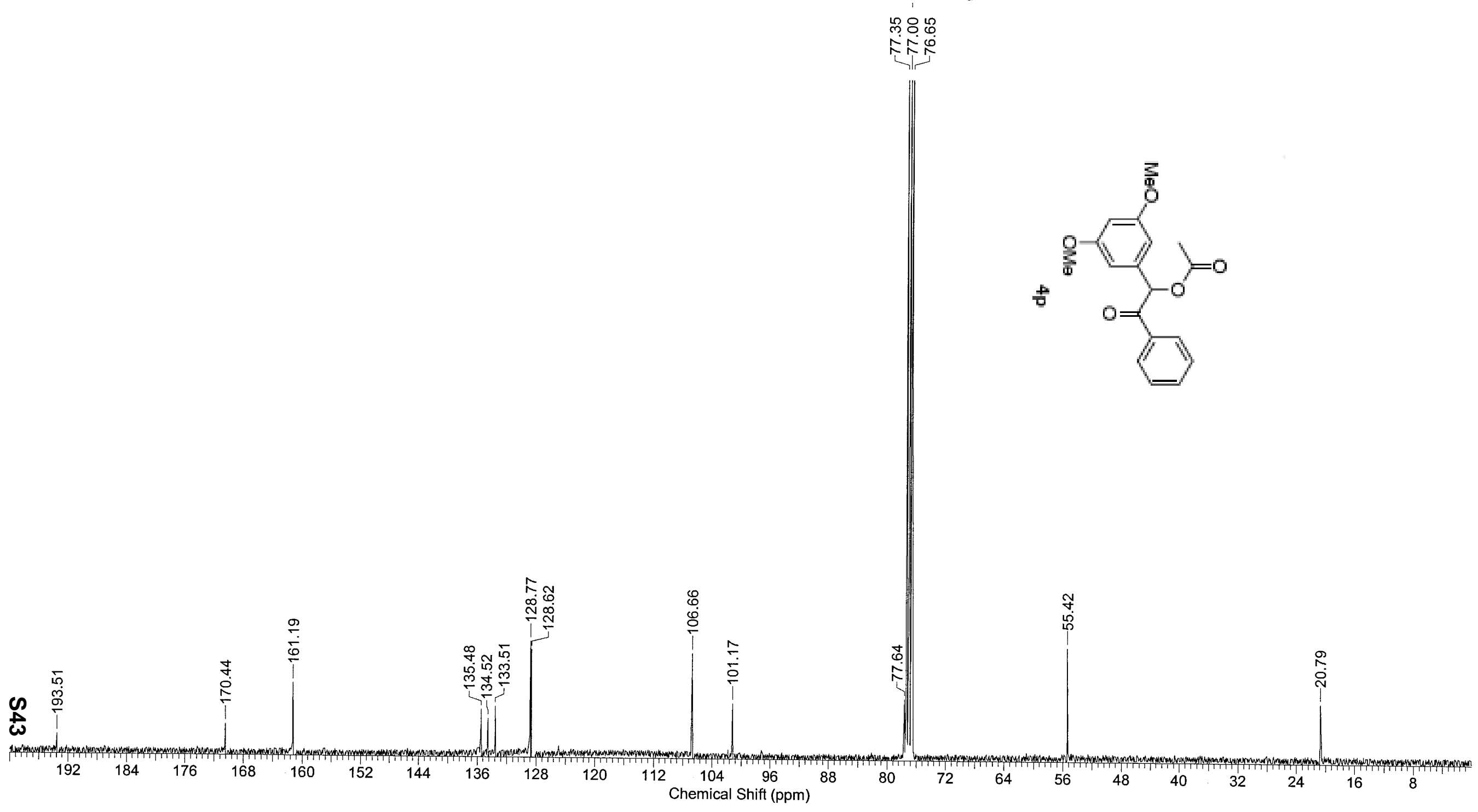




\section{jld47-1}

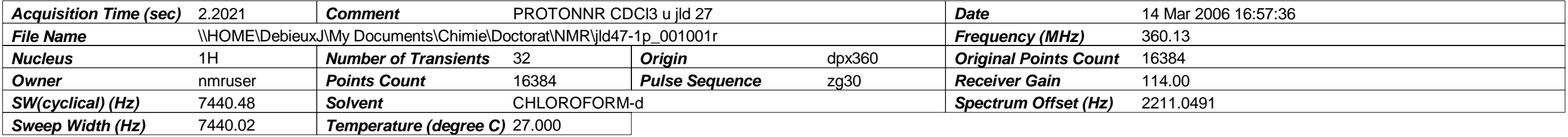

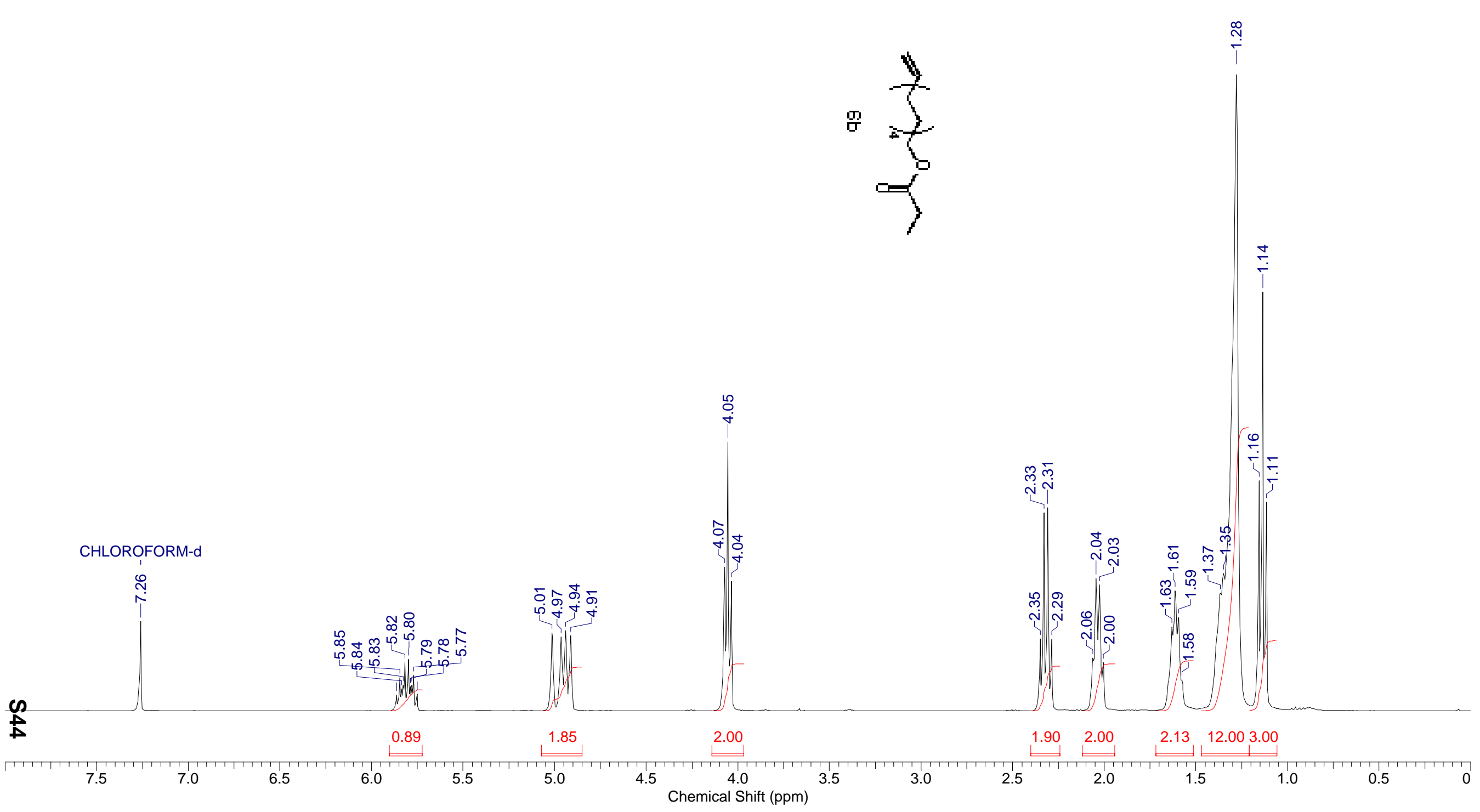




\section{jld47 C13}

\begin{tabular}{|c|c|c|c|c|c|c|c|}
\hline Acquisition Time (sec) & 1.5139 & Comment & \multicolumn{3}{|c|}{ C13CPD CDCl3 u jld 27} & \multicolumn{2}{|c|}{$\begin{array}{ll}\text { Date } & 15 \mathrm{Mar} 2006 \text { 01:21:04 }\end{array}$} \\
\hline File Name & IIHOMEIL & JIMy DocumentsIChimiel & ctoratlN & IC13_001001r & & Frequency $(\mathrm{MHz})$ & 90.55 \\
\hline Nucleus & $13 \mathrm{C}$ & Number of Transients & 4096 & Origin & $\mathrm{dp} \times 360$ & Original Points Count & 32768 \\
\hline Owner & nmruser & Points Count & 32768 & Pulse Sequence & zgpg30 & Receiver Gain & 2048.00 \\
\hline SW(cyclical) $(\mathrm{Hz})$ & 21645.02 & Solvent & CHLOF & & & Spectrum Offset $(\mathrm{Hz})$ & 9014.8154 \\
\hline
\end{tabular}

\begin{tabular}{|lll} 
Sweep Width $(\mathrm{Hz})$ & 21644.36 & Temperature (degree C) 27.000 \\
\hline
\end{tabular}

CHLOROFORM-d
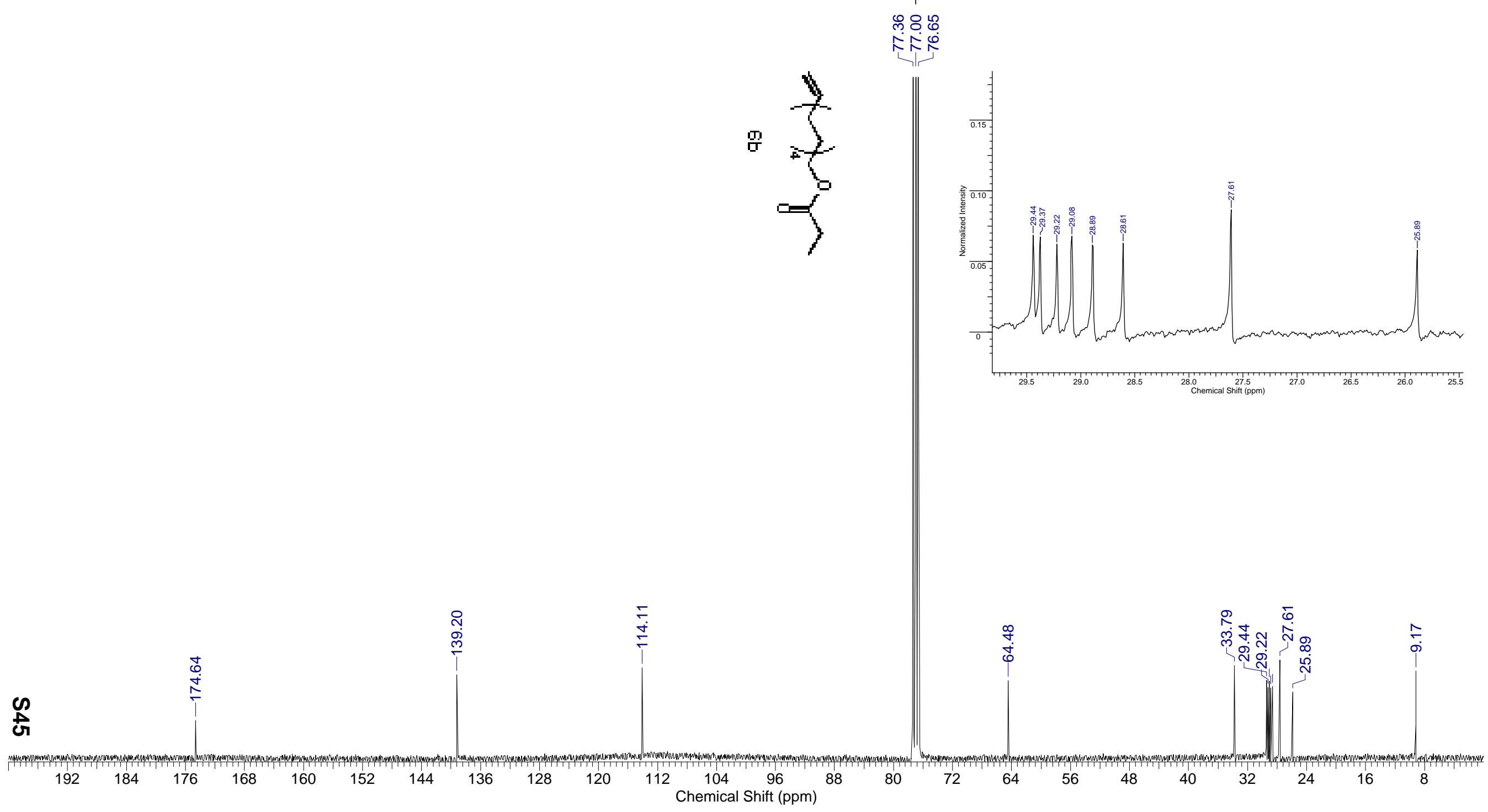
jld59-1

\begin{tabular}{|c|c|c|c|c|c|c|c|}
\hline Acquisition Time (sec) & 2.2021 & Comment & \multicolumn{3}{|c|}{ PROTONNR CDCl3 u jld 59} & Date & 25 Apr 2006 12:52:16 \\
\hline File Name & \multicolumn{5}{|c|}{ IIHOMEIDebieuxJIMy DocumentsIChimielDoctorat|NMRIjld59-1p_001001r } & Frequency (MHz) & 360.13 \\
\hline Nucleus & $1 \mathrm{H}$ & Number of Transients & 16 & Origin & dpx360 & Original Points Count & 16384 \\
\hline Owner & nmruser & Points Count & 16384 & Pulse Sequence & zg30 & Receiver Gain & 71.80 \\
\hline SW(cyclical) $(\mathrm{Hz})$ & 7440.48 & Solvent & $\mathrm{CHLOF}$ & & & Spectrum Offset $(\mathrm{Hz})$ & 2210.1406 \\
\hline
\end{tabular}

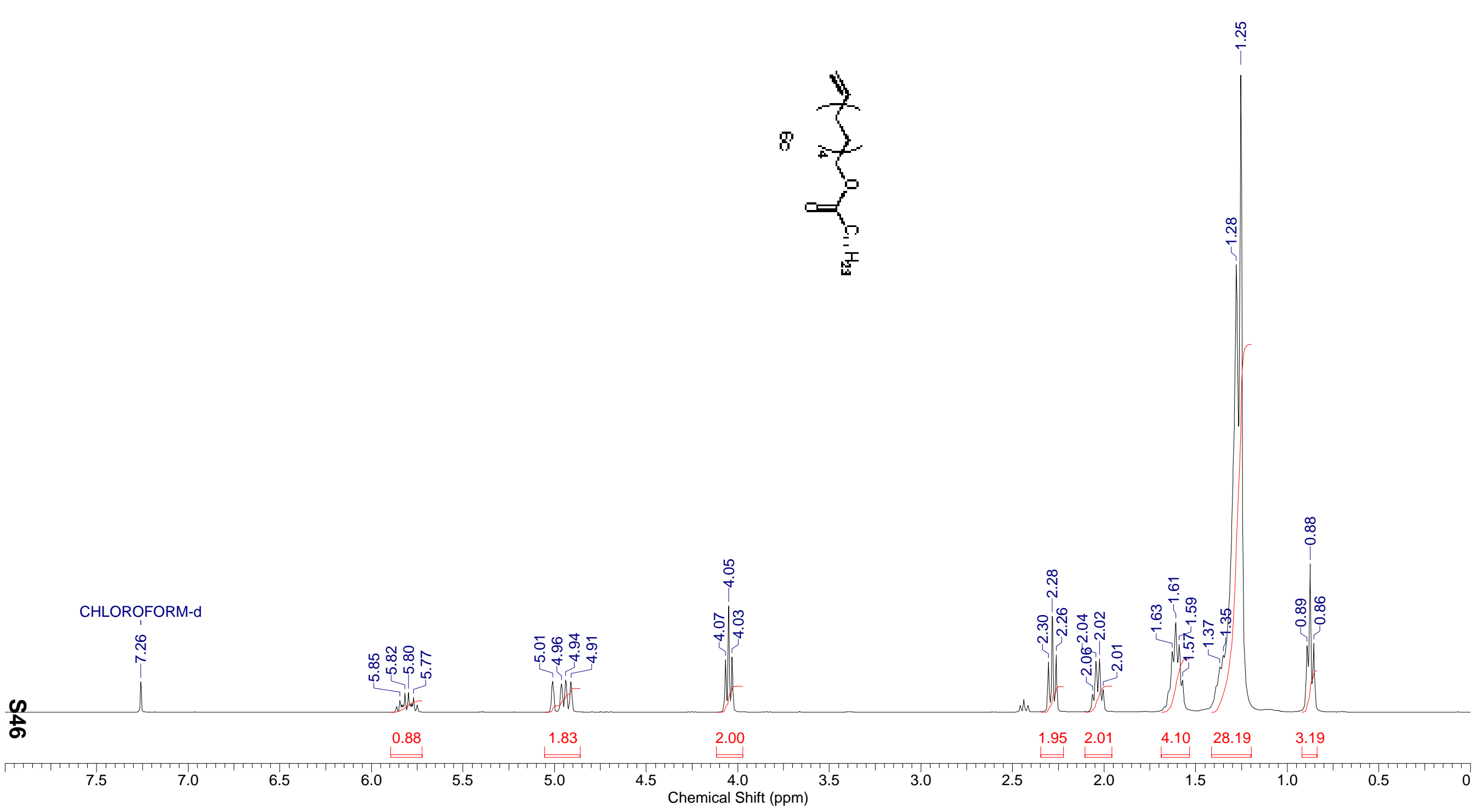




\section{jld59, C13}

\begin{tabular}{|c|c|c|c|c|c|c|c|}
\hline A cquisition $\mathrm{Tm}$ e (sec) & 1.5139 & \multicolumn{4}{|c|}{ Com m ent $\quad$ C13CPD CDCl3 u jld 59} & Date & 25 Apr 2006 22:06:56 \\
\hline File $\mathrm{N}$ am e & \multicolumn{5}{|c|}{ IIHOMEIDebieuxJIMy DocumentsIChimielDoctoratINMRIjld59-1_C13_001001r } & Frequency $\mathrm{MHz}$ ) & 90.55 \\
\hline Nucleus & $13 \mathrm{C}$ & Num beroftransients & 4096 & origin & $d p \times 360$ & O riginalPoints Count & 32768 \\
\hline Owner & nmruser & Points Count & 32768 & Pulse Sequence & zgpg30 & ReceiverG ain & 1625.50 \\
\hline SW (cyclical) (Hz) & 21645.02 & \multicolumn{4}{|c|}{ CHLOROFORM-d } & Spectrum $O$ ffset $(H z)$ & 9016.1367 \\
\hline
\end{tabular}

CHLOROFORM-d

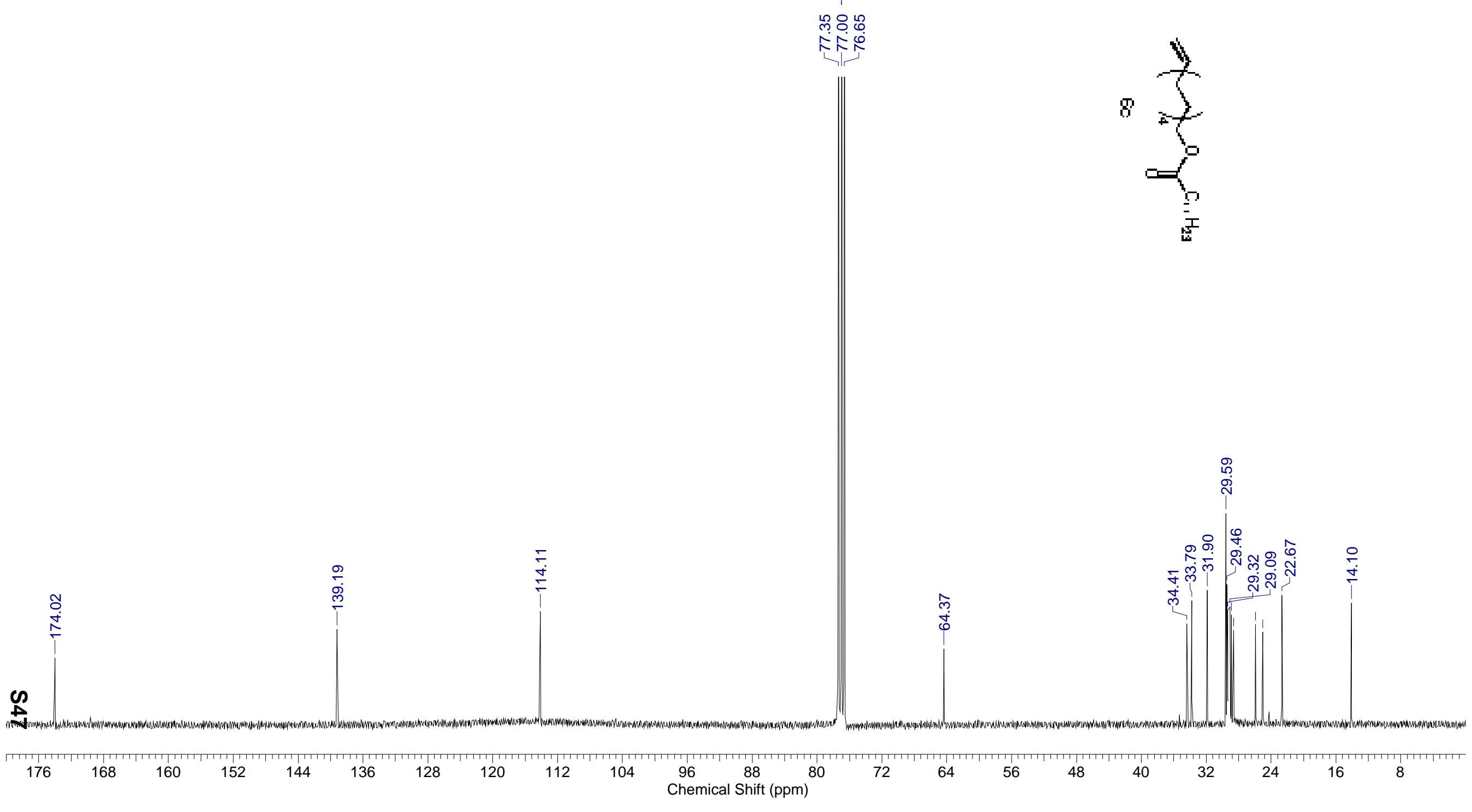




\section{jld48}

\begin{tabular}{|c|c|c|c|c|c|c|c|}
\hline Acquisition Time (sec) & \multicolumn{5}{|c|}{\begin{tabular}{l|ll}
2.2021 & Comment & PROTONNR CDCl3 u jld 48
\end{tabular}} & Date & 17 Mar 2006 13:09:20 \\
\hline File Name & \multicolumn{5}{|c|}{ IIHOMEIDebieuxJIMy DocumentsIChimielDoctoratINMRIjld48-1p_001001r } & Frequency $(\mathrm{MHz})$ & 360.13 \\
\hline Nucleus & $1 \mathrm{H}$ & Number of Transients & 32 & Origin & $\mathrm{dp} \times 360$ & Original Points Count & 16384 \\
\hline Owner & nmruser & Points Count & \multirow{2}{*}{\multicolumn{3}{|c|}{\begin{tabular}{l|l}
16384 & Pulse Sequence \\
CHLOROFORM-d
\end{tabular}}} & Receiver Gain & 90.50 \\
\hline SW(cyclical) $(\mathrm{Hz})$ & 7440.48 & Solvent & & & & Spectrum Offset $(\mathrm{Hz})$ & 2210.5947 \\
\hline Sweep Width $(\mathrm{Hz})$ & 7440.02 & \multicolumn{4}{|c|}{ Temperature (degree $C$ ) 27.000} & & \\
\hline
\end{tabular}

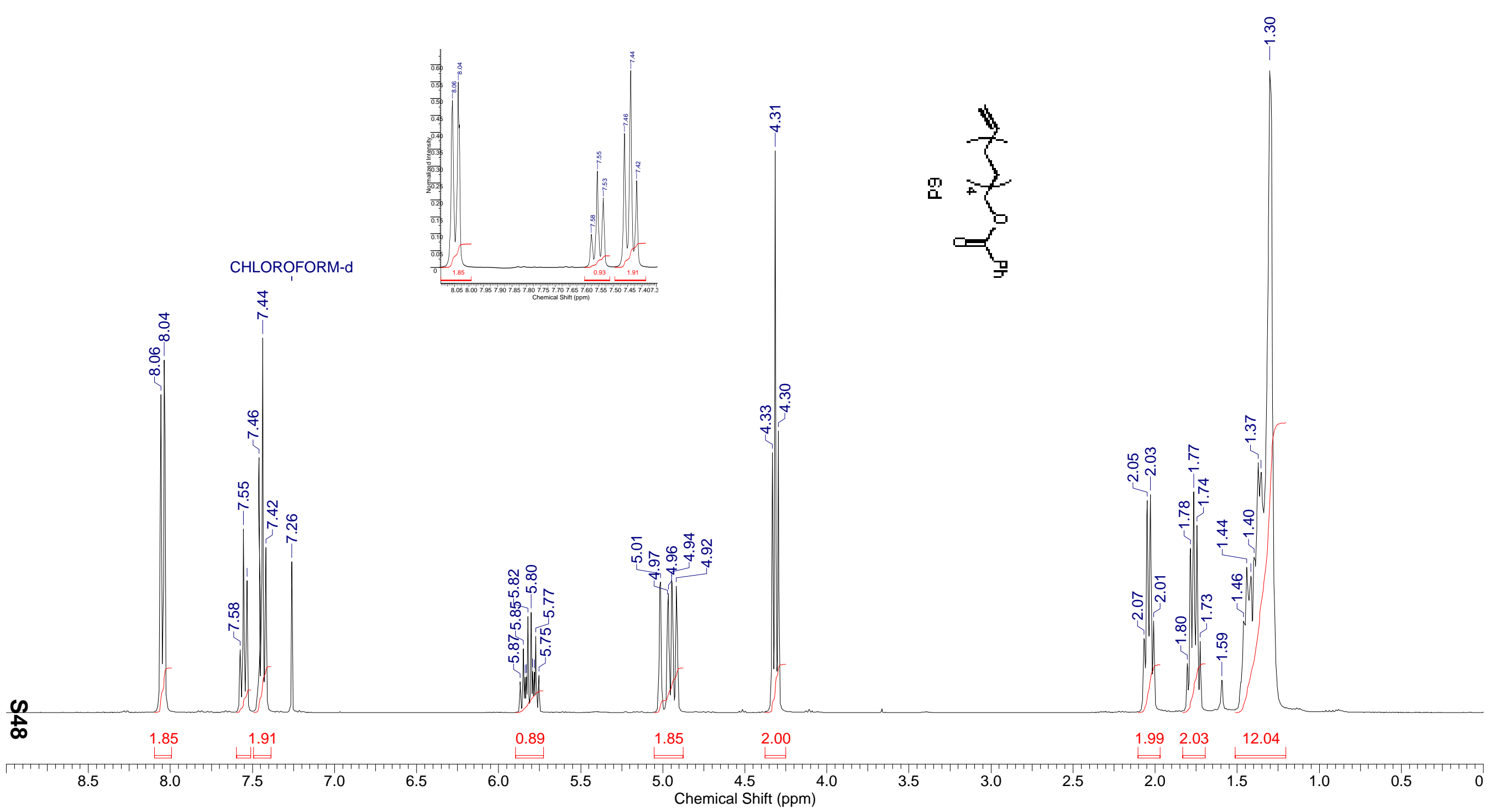




\section{jld48 C13}

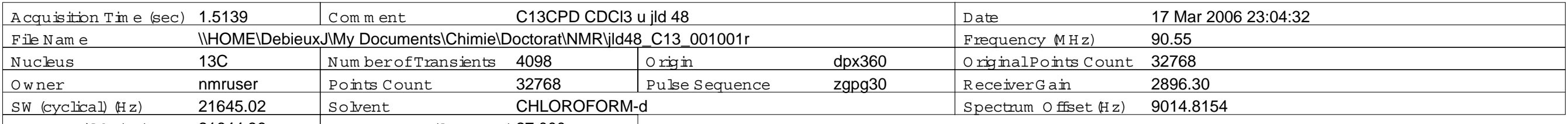

$\begin{array}{lll}\mathrm{S} w \text { eep W idth }(\mathrm{H} z) & 21644.36 & \text { Tem perature (degree C) } 27.000\end{array}$

CHLOROFORM-d
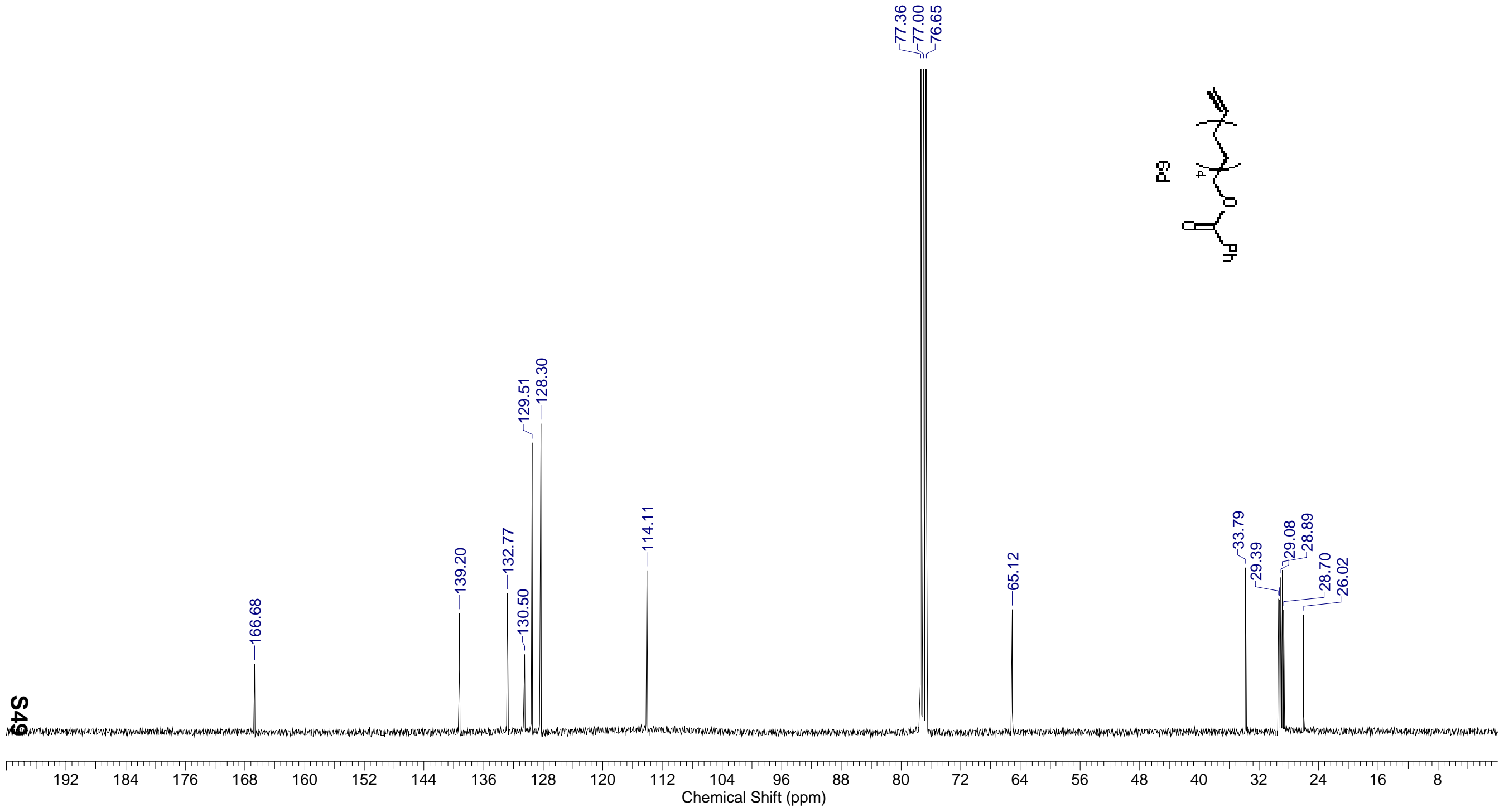


\section{jld58}

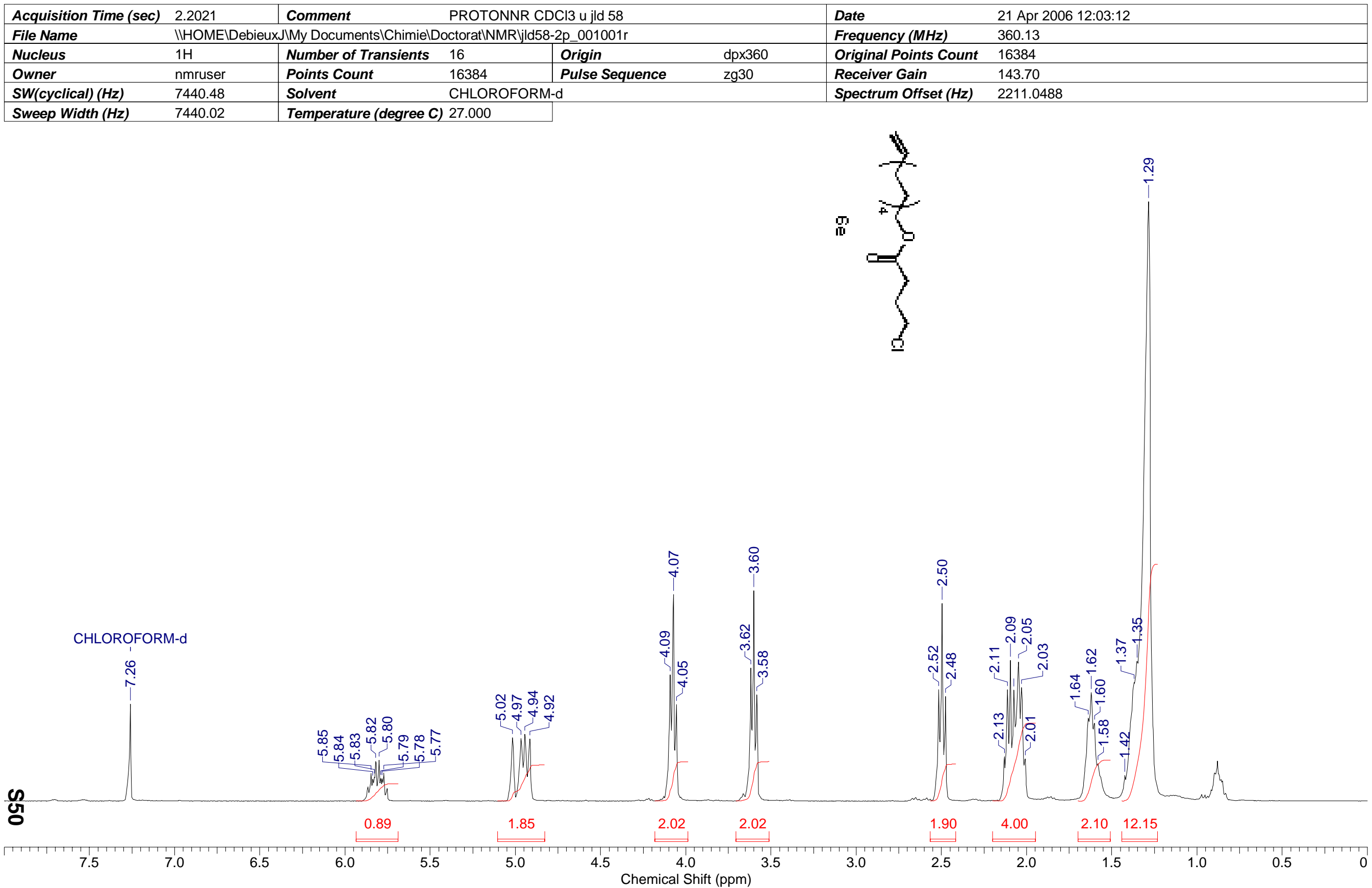




\section{jld58, C13}

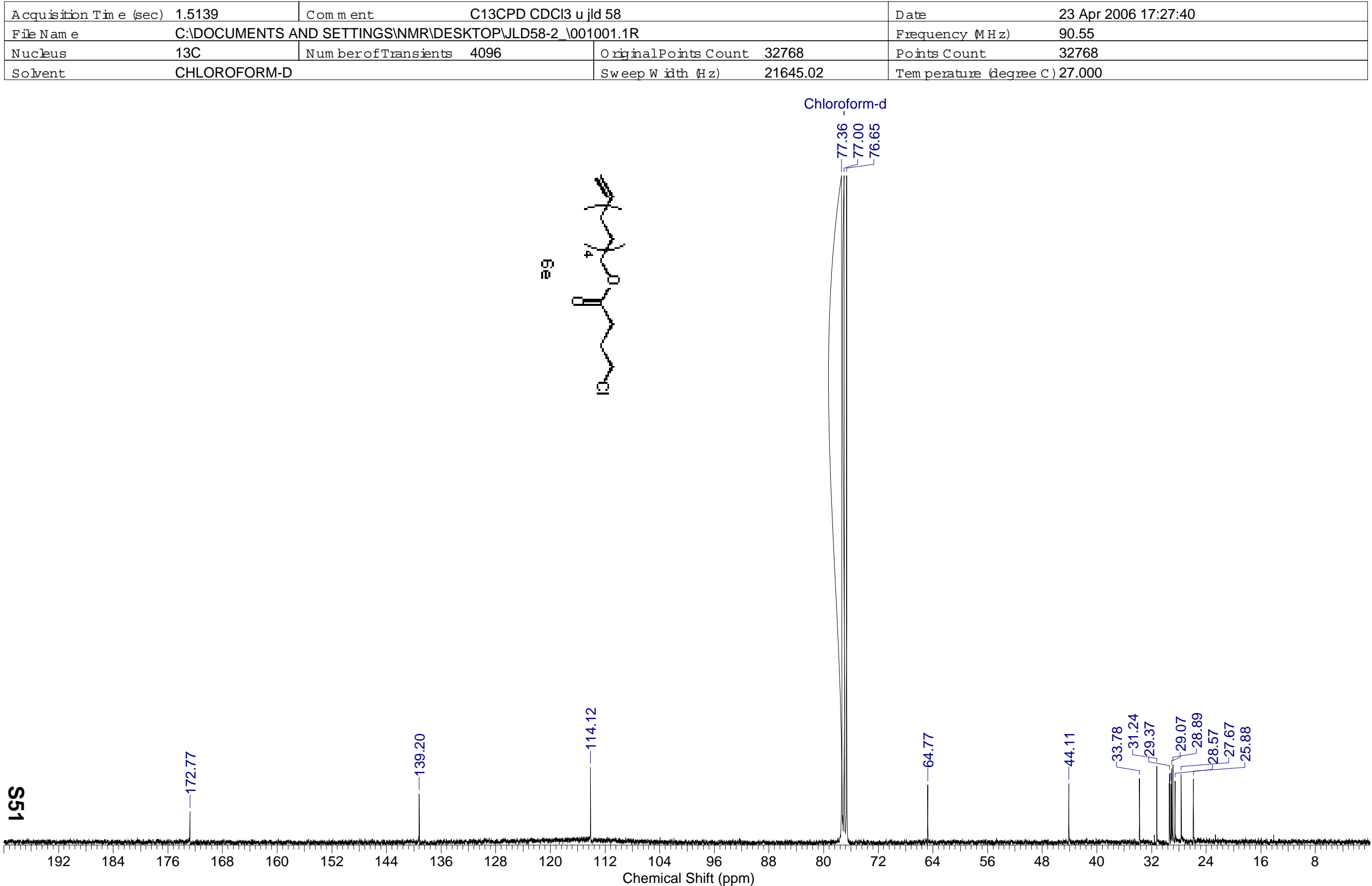




\section{jld60}

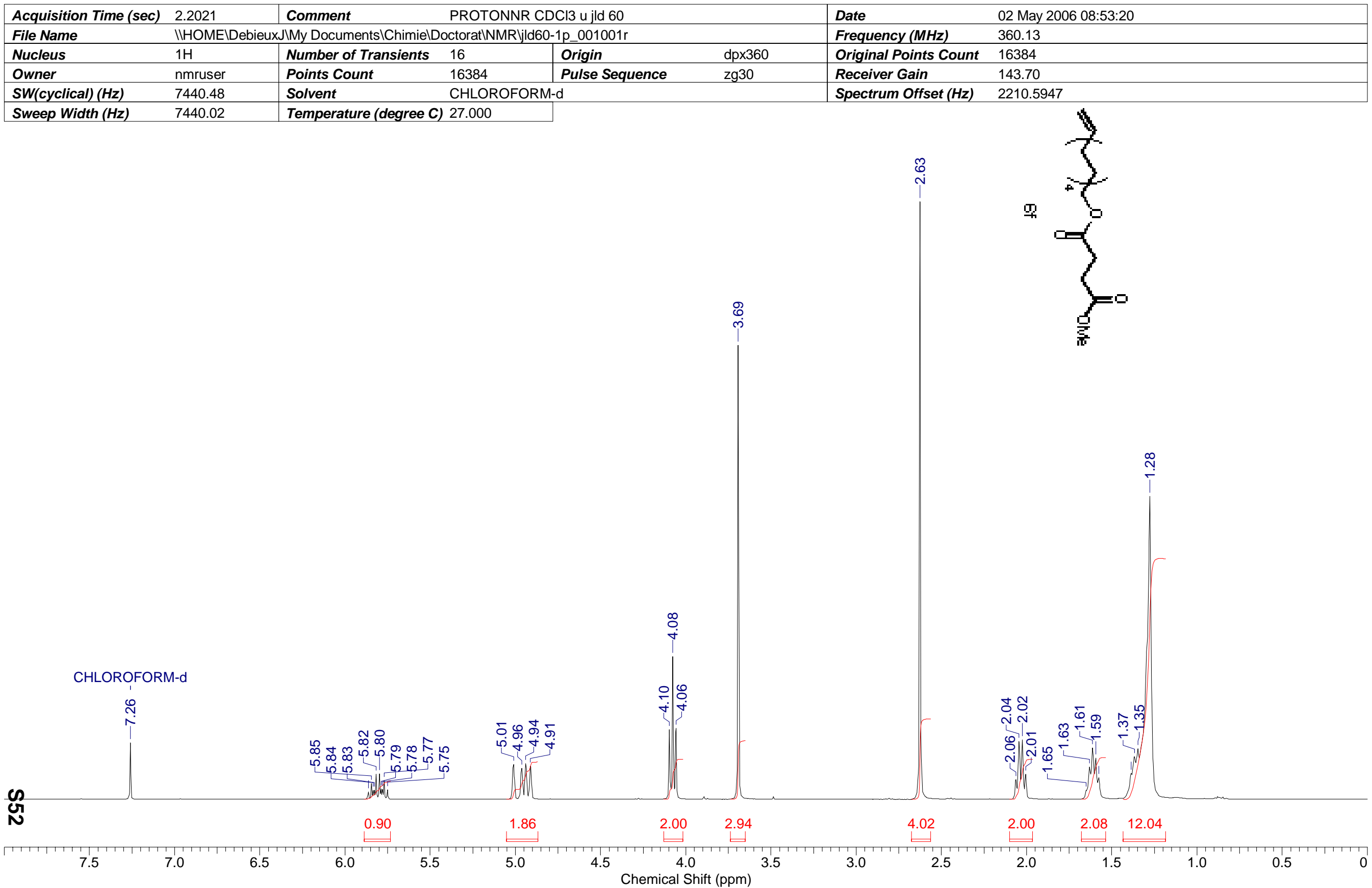




\section{jld60, C13}

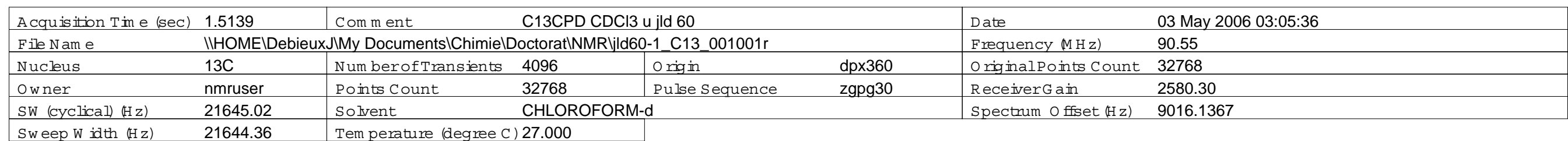

\section{CHLOROFORM-d}

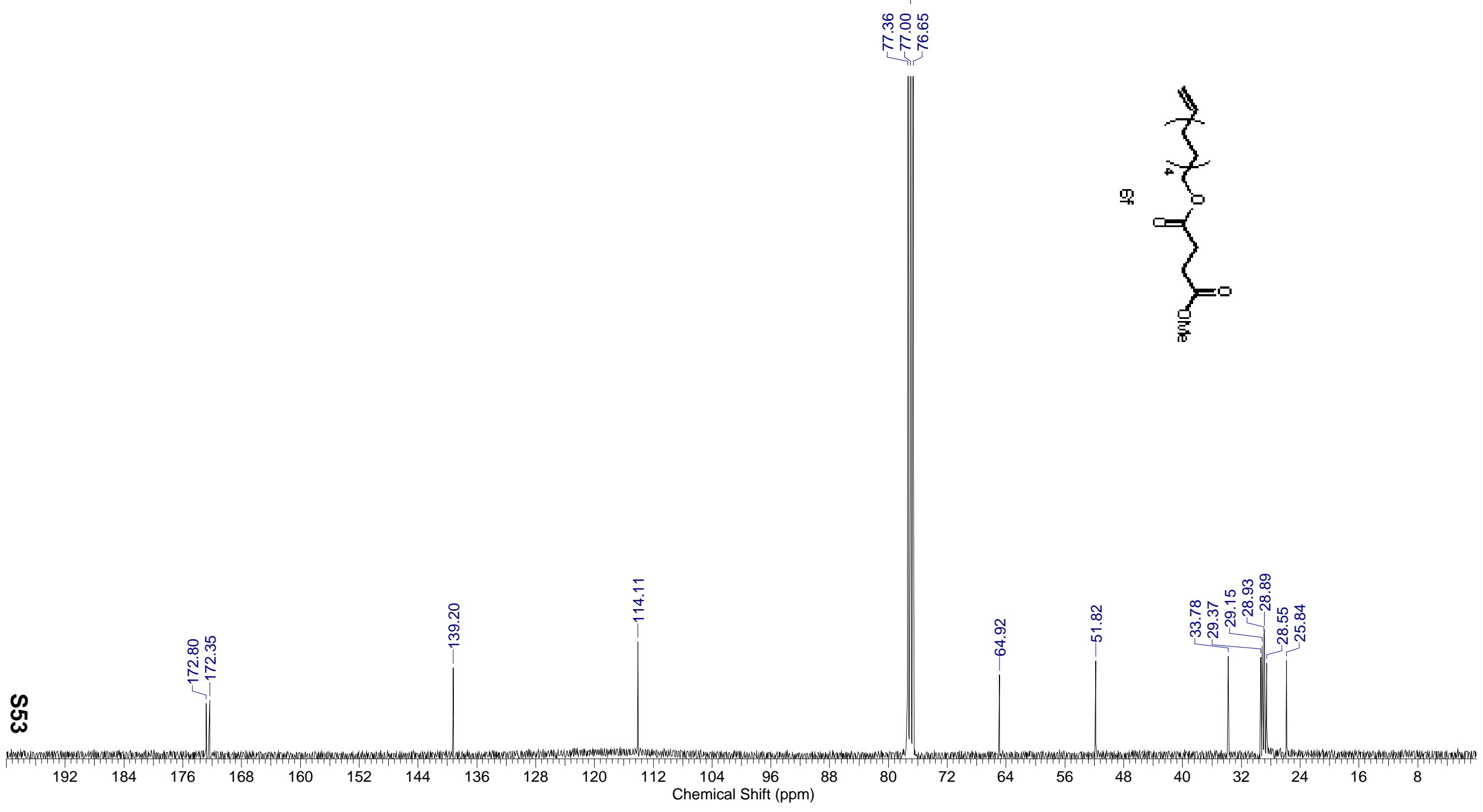

